UNIVERSIDADE DE BRASÍLIA

FACULDADE DE TECNOLOGIA

DEPARTAMENTO DE ENGENHARIA MECÂNICA

PROJETO DE GRADUAÇÃO

\title{
ANÁLISE EXERGÉTICA DE CICLOS COMBINADOS
}

ALUNO: DIEGO NEVES KALATALO

ORIENTADOR: JOSÉ LUIZ ALVES FONTOURA RODRIGUES 
UNIVERSIDADE DE BRASÍLIA

Faculdade de Tecnologia

Departamento de Engenharia Mecânica

\author{
PROJETO DE GRADUAÇÃO
}

ANÁLISE EXERGÉTICA DE CICLOS COMBINADOS

POR

Diego Neves Kalatalo

Projeto submetido como requisito parcial para obtenção do grau de Engenheiro Mecânico

\title{
ORIENTADOR:
}

José Luiz Alves Fontoura Rodrigues 


\section{AGRADECIMENTOS}

Aqui presto meus agradecimentos às pessoas e instituições que contribuíram para a realização deste trabalho. Dentre elas cito:

Afonso e Alzira, meus pais e orientadores para a vida.

Francisdalva e Lara, minha esposa e filha, pela motivação que representam para minha existência.

Professor José Luiz Alves, por ter me dado a honra e oportunidade de trabalhar ao seu lado.

Ao funcionário Sérgio da usina de Uruguaiana, pela grande contribuição com as informações a mim disponibilizadas.

Aos meus amigos de trabalho, pelo incentivo e tolerância em relação às minhas ausências no trabalho.

Às Universidades de Viçosa e de Brasília. 


\section{RESUMO}

Este trabalho pretende a realização de uma análise exergética de um ciclo combinado em operação no Brasil. Para atingir o resultado proposto será desenvolvida a análise de cada componente do ciclo de acordo com o conceito de exergia baseado na $1^{\mathrm{a}}$ e na $2^{\mathrm{a}}$ lei da termodinâmica. Após a determinação da exergia, será possível a avaliar e quantificar as perdas associadas às irreversibilidades dos possíveis processos de conversão de energia em trabalho útil. O estudo desenvolvido poderá ser aplicado à ciclos similares em operação no país e fornecer importantes informações a respeito do gerenciamento da energia disponibilizada pela termogeração no Brasil.

\section{ABSTRACT}

This work aims to conduct an analysis of an exergetic combined cycle operating in Brazil. To achieve the result proposed will be developed analysis of each component lifecycle in accordance with the concept of exergy based on 1st and 2nd law of thermodynamics. After the determination of exergy, it will be possible to evaluate and quantify the losses associated with irreversibilities of potential energy conversion processes on net work. The study developed can be applied to similar cycles in operation in the country and provide important information about the management of the energy released by thermal generation in Brazil. 
1 INTRODUÇÃO …............................................................................................ 1

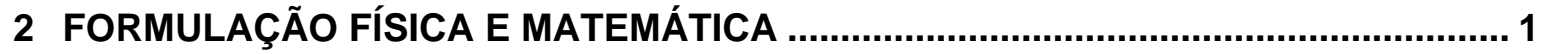

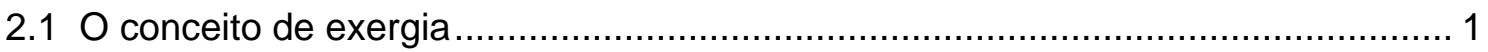

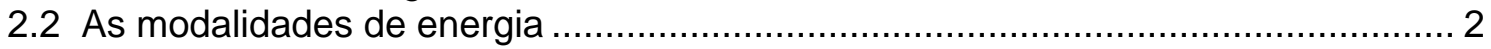

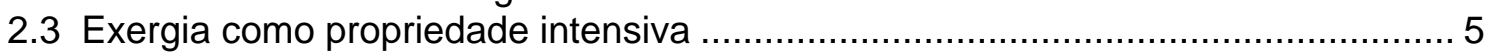

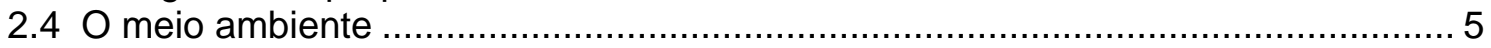

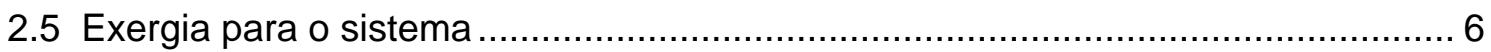

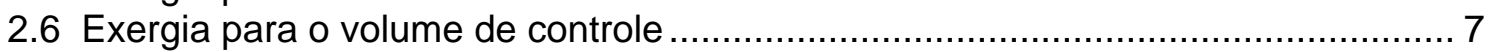

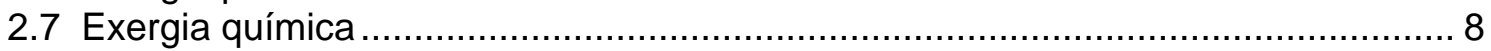

2.8 Matéria prima, produtos e perdas ................................................................. 9

2.9 Eficiência exergética e Eficiência exergética maior ............................................ 9

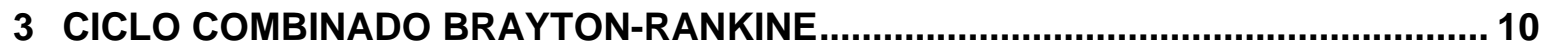

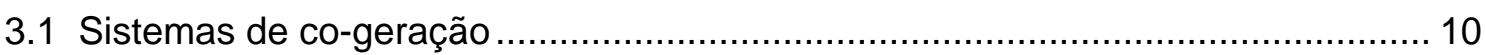

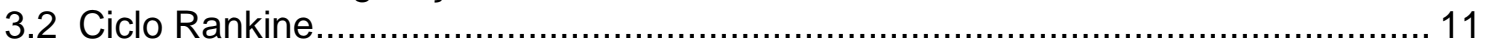

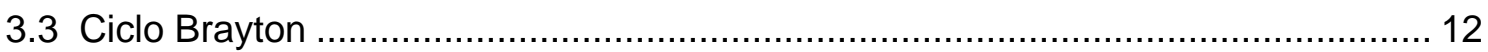

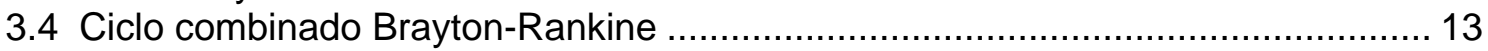

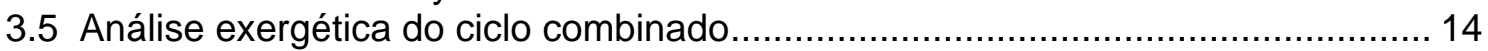

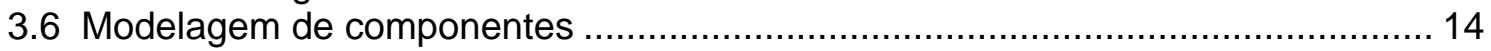

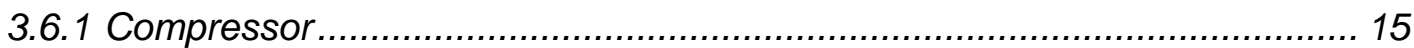

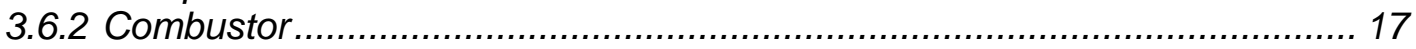

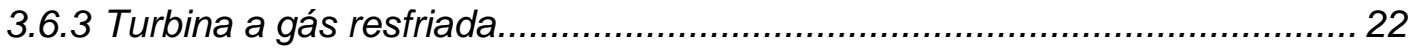

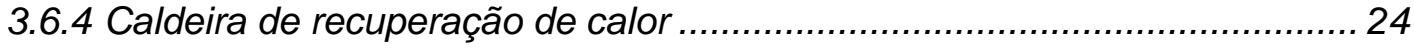

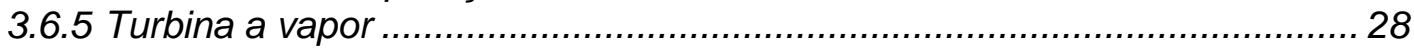

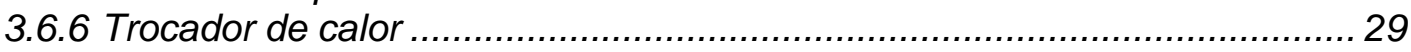

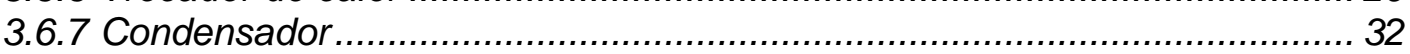

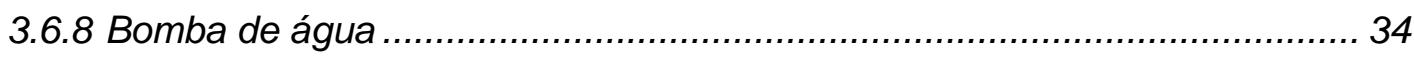

4 ANÁLISE DA USINA TERMELÉTRICA DE URUGUAIANA .................................... 36

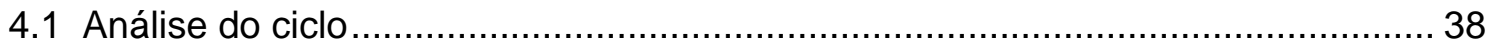

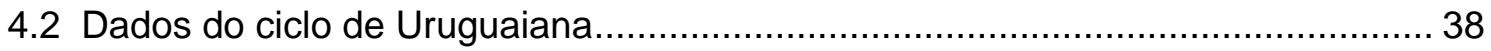

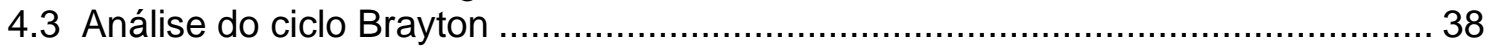

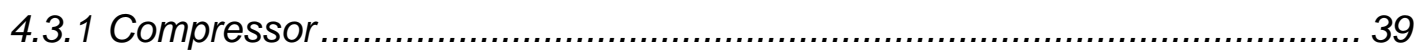

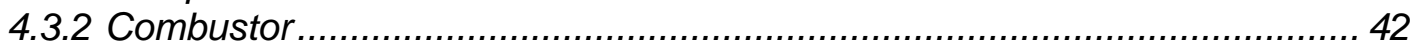

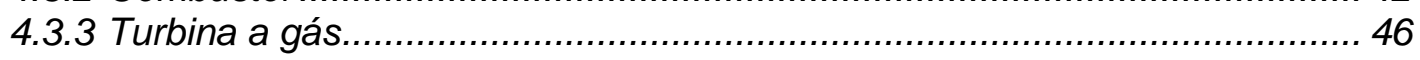

4.4 Análise do ciclo Rankine........................................................................ 50

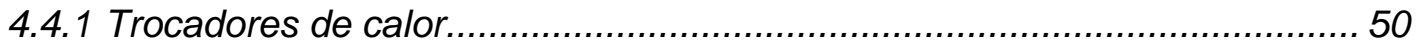

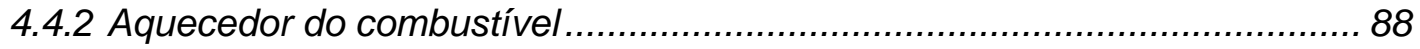

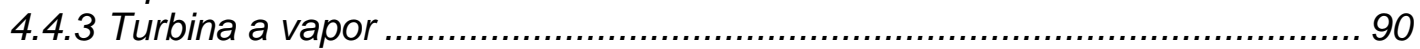

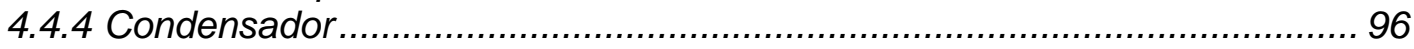

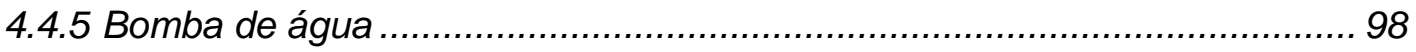

4.5 Síntese da análise do ciclo de Uruguaiana .............................................. 104

4.5.1 Eficiência total do ciclo .................................................................... 104

4.6 Representação do ciclo por diagramas .................................................... 105

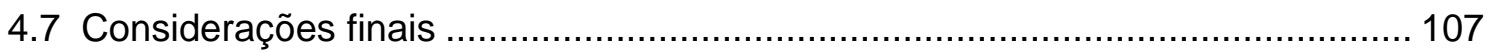


5 CONCLUSÕES

108

6 REFERÊNCIAS BIBLIOGRÁFICAS..

109

A1 - ALGORITMO PARA CÁLCULO DA TEMPERATURA DOS GASES NA SAÍDA DO COMBUSTOR. 


\section{ÍNDICE DE FIGURAS}

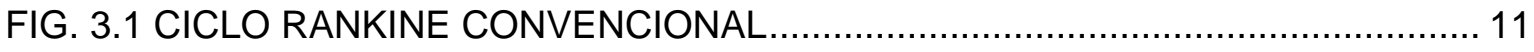

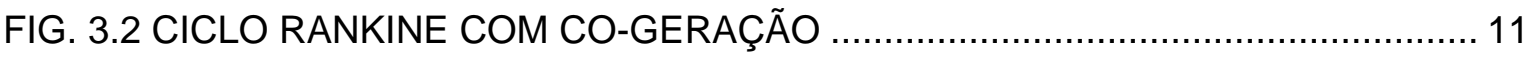

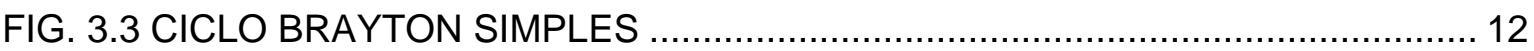

FIG. 3.4 CICLO BRAYTON SIMPLES COM CO-GERAÇÃO ........................................ 12

FIG. 3.5 CICLO COMBINADO COM CO-GERAÇÃO ….............................................. 13

FIG. 3.6 ESQUEMA DE UMA CENTRAL A CICLO COMBINADO GÁS/VAPOR ............. 14

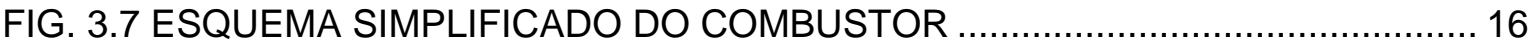

FIG. 3.8 ESQUEMA DO COMBUSTOR - MODELAGEM …..................................... 17

FIG. 3.9 VOLUME DE CONTROLE PARA COMBUSTOR - ANÁLISE EXERGÉTICA .... 20

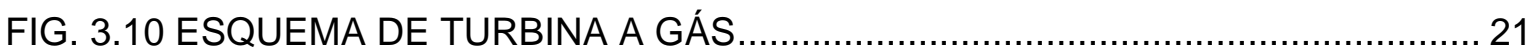

FIG. 3.11 ESQUEMA DE CALDEIRA DE RECUPERAÇÃO DE CALOR ….................... 24

FIG. 3.12 PERFIL DE TEMPERATURAS NA CALDEIRA DE RECUPERAÇÃO DE

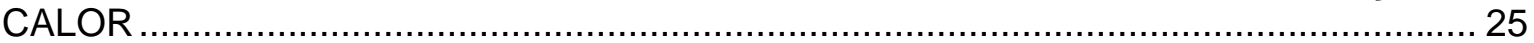

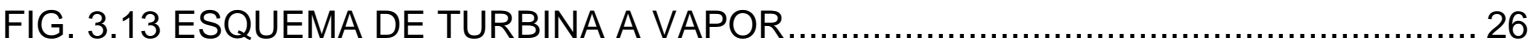

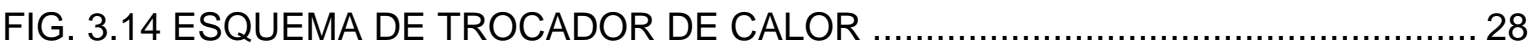

FIG. 3.15 TEMPERATURA DOS FLUIDOS NO TROCADOR DE CALOR …................... 29

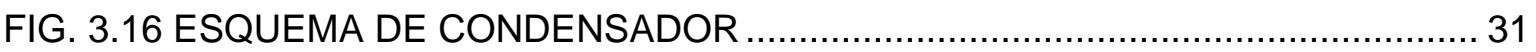

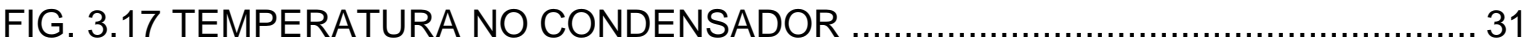

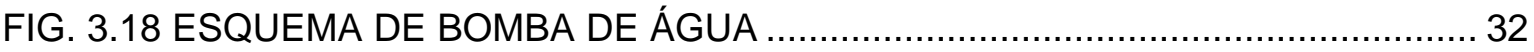

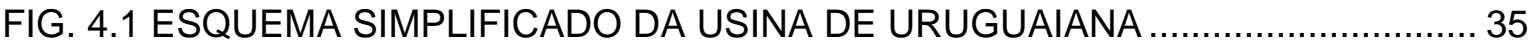

FIG. 4.2 ESQUEMA DO CORREDOR DE PASSAGEM DOS GASES AQUECIDOS ...... 48

FIG. 4.3 DIAGRAMA DE GRASMAM PARA A USINA DE URUGUAIANA (CICLO

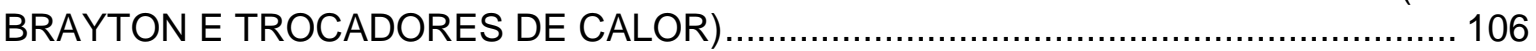

FIG. 4.4 DIAGRAMA DE GRASMAM PARA A USINA DE URUGUAIANA (TURBINAS A VAPOR E CONDENSADOR). 
ÍNDICE DE TABELAS

TABELA 4.1 SÍNTESE DA ANÁLISE EXERGÉTICA DO CICLO DE URUGUAIANA .... 104 


\section{NOTAÇÕES}

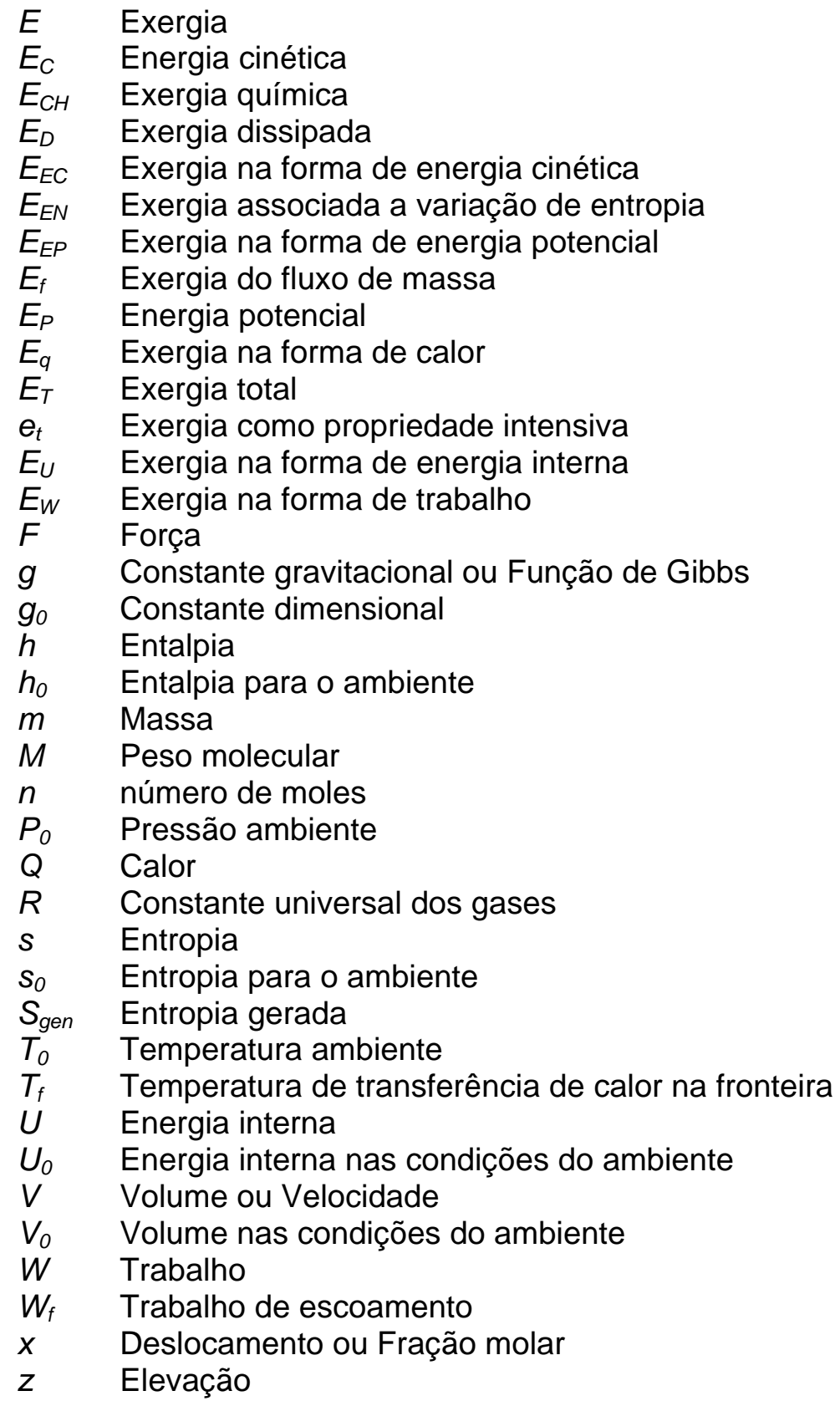

\section{Símbolos Gregos}

$\begin{array}{ll}\Sigma & \text { Somatório } \\ \eta & \text { Eficiência (energética, isoentrópica ou politrópica) } \\ \mu & \text { Potencial químico } \\ \lambda & \text { Relação ar/combustível } \\ \varepsilon & \text { Eficiência exergética } \\ \varepsilon^{+} & \text {Eficiência exergética maior }\end{array}$




\section{Índices}

$\begin{array}{ll}\text { ad } & \text { Ar admitido } \\ \text { am } & \text { Ar de mistura } \\ b \text { ou } & \text { Bomba } \\ c & \text { Energia cinética } \\ c b & \text { Relativo ao combustível } \\ c c & \text { Combustor } \\ C p & \text { Compressor ou pressão constante } \\ D \text { ou } & d \text { Dissipação de exergia } \\ e & \text { Entrada } \\ \text { ec } & \text { Economizador } \\ g & \text { Gases } \\ g v & \text { Gerador de vapor } \\ g r & \text { Gases da reação } \\ p & \text { Energia ou exergia potencial } \\ \text { iso } & \text { Isoentrópico } \\ l & \text { Perda de exergia } \\ \text { Is } & \text { Líquido saturado } \\ m & \text { Matéria prima } \\ p & \text { Produtos } \\ p o l i & \text { Politrópico } \\ s & \text { Saída } \\ \text { sa } & \text { Superaquecedor } \\ t g & \text { Turbina a gás } \\ t v & \text { Turbina a vapor } \\ v \text { ou } & v p \text { Vapor }\end{array}$




\subsection{Circunstâncias}

A energia elétrica produzida no Brasil é, em grande parte, resultado de geração hidroelétrica. Segundo dados do SIPOT, Sistema de Informações do Potencial Hidrelétrico Brasileiro, em dezembro de 2.000 o potencial hidrelétrico brasileiro foi estimado em $260.096 \mathrm{MW}$, entretanto somente $25 \%$ desse potencial vem sendo utilizado pelas usinas atualmente em operação.

Em decorrência da opção pela privatização do setor energético, durante o governo Fernando Henrique Cardoso, o planejamento para atender as demandas de energia foi abandonado, resultando no colapso do fornecimento de energia e desencadeando em 2001 uma crise no sistema energético que culminou com o racionamento de energia elétrica entre junho de 2001 e fevereiro de 2002.

Diante disso, fez-se necessária uma solução imediata a fim de suprir as deficiências de atendimento dos consumidores, por meio de alternativas de geração de energia elétrica com prazo de implantação reduzido.

A melhor alternativa disponível é a geração de energia elétrica a partir de usinas termelétricas de ciclo combinado. Dentre as vantagens deste tipo de sistema, destaca-se o curto prazo para implantação e alta flexibilidade para atendimento de cargas de ponta.

Os ciclos combinados modernos são disponibilizados em uma ampla faixa de capacidades, com módulos de $2 \mathrm{MW}$ até $800 \mathrm{MW}$, apresentando rendimentos térmicos próximos de $60 \%$. Estudos prospectivos prevêem que, por meio de desenvolvimento tecnológico em curso, o rendimento térmico destes ciclos possa atingir até $70 \%$, em um período relativamente curto. Tal perspectiva baseia-se na possibilidade de que os ciclos de potência passem a operar com temperaturas máximas mais elevadas, da ordem de $1600^{\circ} \mathrm{C}$, em substituição das temperaturas máximas atuais que oscilam entorno de $1450^{\circ} \mathrm{C}$.

\subsection{Objetivo do trabalho}

Considerando a falta de tradição no trato com a tecnologia de ciclos combinados no Brasil e a conseqüente carência de pessoal técnico de nível superior, preparado para trabalhar nesta área, o objetivo deste trabalho é introduzir o aluno executor no estudo termodinâmico de ciclos combinados.

A metodologia a ser adotada pretende a realização da análise exergética de um ciclo combinado completo, tendo como caso teste uma usina já em funcionamento no Brasil. Para este trabalho será analisada a usina termelétrica de Uruguaiana localizada no Rio Grande do Sul.

\subsection{Resultados a serem atingidos}

Calcular a exergia total associada a um ciclo combinado em operação, por meio das análises de desempenho de cada componente do ciclo combinado, determinando a disponibilidade de energia proporcionada pelo ciclo. existentes.

Com base no desempenho operacional do ciclo Identificar e quantificar as perdas

\section{Formulação física e matemática}

\subsection{O conceito de exergia}

Os métodos tradicionais de análise de ciclos térmicos, baseados na $1^{\text {a }}$ Lei da Termodinâmica, são capazes de quantificar os fluxos de calor e trabalho que se estabelecem nestas circunstâncias. Entretanto, esta modalidade de balanço energético, não fornece informações sobre a disponibilidade da energia ou sobre as perdas internas ocorridas. Em contrapartida, se a $2^{\mathrm{a}}$ Lei da Termodinâmica for acrescentada à análise, cria-se a base teórica necessária para introduzir um conceito, largamente utilizado na análise e otimização de ciclos térmicos, denominado de exergia ou de disponibilidade. 
A exergia ou disponibilidade de um sistema, em um estado termodinâmico de referência, pode ser definida como a parcela da energia total do sistema que pode ser convertida em trabalho, por meio de um processo ideal capaz de levar o sistema do estado termodinâmico de referência até o estado de completo equilíbrio com o meio ambiente.

\subsection{As modalidades de energia}

Sendo a exergia caracterizada a partir da energia total do sistema, é conveniente a classificação das diferentes modalidades de energia, que compõem a energia total, tomando como critério as formas e peculiaridades da transformação, das diferentes modalidades de energia, em trabalho. Neste estudo, serão estudadas formas de energia presentes em sistemas térmicos destinados à geração de energia elétrica, assim a análise será restrita às modalidades de energia associadas a tais sistemas.

\subsubsection{Trabalho}

A definição clássica de trabalho é dada pelo produto de uma força $F$ por um deslocamento $x$, sendo este último na mesma direção da força. $\mathrm{O}$ trabalho $W$ pode então ser expresso por,

$$
W=\int F d x .
$$

Utilizando-se desta equação, pode-se calcular o trabalho necessário para levantar um peso, esticar um fio ou mover uma partícula carregada através de um campo magnético.

Entretanto, como o interesse do trabalho é a análise exergética de sistemas térmicos, é vantajoso relacionar a definição de trabalho com o conceito de exergia e ambiente. Define-se, portanto, o trabalho como sendo a modalidade de energia que pode ser representada pelo movimento de um eixo rotativo de uma turbina, movimento de eixo de um compressor axial, ação de uma bomba centrífuga ou o movimento da fronteira do sistema. Este último deve ser considerado com algum detalhe, pois o mesmo está diretamente associado às condições do ambiente e será alterado sempre que essas condições forem alteradas. Tal trabalho é realizado para alterar o volume $V$ do sistema contra a pressão do ambiente $P_{0}$, até atingir-se um volume $V_{0}$, em equilíbrio com o ambiente. Este tipo de trabalho é denominado por $W_{f}$ e pode ser calculado pela relação

$$
W_{f}=p_{0}\left(V_{0}-V\right) .
$$

Definindo qualquer forma de trabalho presente no sistema em análise por $W$ e considerando o trabalho realizado contra a fronteira, têm-se a definição de exergia para a modalidade de energia, $E_{W}$, na forma de trabalho dada por,

$$
E_{W}=W-W_{f} .
$$

\subsubsection{Calor}

O calor é definido como sendo a forma de energia transferida através da fronteira de um sistema numa dada temperatura, a um outro sistema ou ao meio ambiente, numa temperatura inferior, em virtude da diferença de temperatura entre os dois sistemas. Definindo esta modalidade de energia de transição pelo símbolo $Q$, pode-se expressar a exergia associada aos fluxos de calor, $E_{q}$, pela equação, 


$$
E_{q}=\left(1-\frac{T_{0}}{T_{f}}\right) Q
$$

Neste caso a exergia é quantificada como o trabalho que seria gerado por uma máquina de Carnot operando entre a temperatura do ambiente $T_{0}$ e a temperatura na qual o fluxo de calor é transferido, $T_{f}$.

\subsubsection{Energia Potencial}

A energia potencial está associada ao sistema de coordenadas escolhido e pode ser determinada pelos parâmetros macroscópicos de massa e elevação. Considerando que a aceleração, devido à gravidade seja $g$, que $m$ represente a massa do sistema e $Z$ sua altura, em relação ao sistema de referência, então a energia potencial $E P$ é expressa pela equação,

$$
E P=\frac{m g Z}{g_{0}} .
$$

onde $g_{0}$ representa a constante dimensional.

Assim, considerando que toda energia potencial pode ser convertida em trabalho sem perdas de qualquer tipo, a exergia associada à energia potencial, $E_{E P}$, é representada pela relação,

$$
E_{E P}=\frac{m g Z}{g_{0}} .
$$

\subsubsection{Energia Cinética} relação,

A energia cinética, $E C$, de um sistema com massa $m$ e velocidade $V$ é representada pela

$$
E C=\frac{m V^{2}}{2 g_{0}} .
$$

Considerando que a energia cinética pode ser totalmente convertida em trabalho, sem perdas de qualquer tipo, têm-se para a exergia correspondente, $E_{E C}$, a equação,

$$
E_{E C}=\frac{m V^{2}}{2 g_{0}} .
$$

\subsubsection{Energia Interna}

Energia cinética e potencial estão associadas ao sistema de coordenadas escolhido e podem ser determinadas pelos parâmetros macroscópicos de massa, velocidade e elevação. A energia interna $U$ inclui todas as outras formas de energia do sistema, entretanto não se pode considerar que a mesma será totalmente convertida em trabalho, assim, a exergia correspondente, deve considerar somente a variação entre dois estados termodinâmicos distintos. Neste caso a exergia associada à variação de energia interna de um sistema, $E_{U}$, entre sua condição inicial $U$ e a condição de equilíbrio com o ambiente, $U_{0}$, é representada por, 


$$
E_{U}=U-U_{0} .
$$

\subsubsection{Energia Química}

A energia química de um sistema torna-se disponível por meio de reações, desta natureza, entre as suas substâncias componentes. Neste trabalho considera-se o potencial químico $\mu i$ de cada substância $i$ presente no sistema e, a partir desta propriedade, calcula-se a energia disponível em cada substância que compõe o sistema. Analogamente à energia interna, a energia química também está associada a uma variação entre dois estados distintos de cada substância, esses estados são representados pelas condições do ambiente e do sistema analisado. Assim pode-se expressar a exergia química, $E_{C H}$, por,

$$
E_{C H}=\sum n_{i}\left(\mu_{i}-\mu_{i 0}\right)
$$

Neste caso $n_{i}$ é a quantidade correspondente a cada substância presente no sistema, dada em base molar ou mássica. O subíndice " $i_{0}$ " indica o potencial químico da substância $i$ correspondente ao estado do ambiente.

\subsubsection{Entropia}

A energia associada a fluxos de calor que se estabelecem, entre o sistema e sua vizinhança, quando sua temperatura $T_{0}$ é suposta constante, pode ser representada por meio da variação de entropia sofrida pelo sistema, uma vez que a entropia $S$ é uma propriedade termodinâmica do sistema relacionada ao tipo de irreversibilidade presente nos processos termodinâmicos que envolvem fluxos de calor comandados por gradientes finitos de temperatura. Esta modalidade de exergia, denominada neste estudo como $E_{E N}$, é definida pela relação

$$
E_{E N}=T_{0}\left(S-S_{0}\right)
$$

onde $S$ e $S_{0}$ representam, respectivamente, as entropias de um estado termodinâmico genérico e a do estado termodinâmico da vizinhança do sistema.

Outra modalidade de variação de exergia, devida a existência de irreversibilidades internas, neste trabalho representada pela variável $E_{D}$, pode ser expressa pela relação

$$
E_{D}=T_{0} S_{\text {gen }},
$$

onde $S_{\text {gen }}$ representa a entropia gerada pelas irreversibilidades internas.

\subsection{Exergia como propriedade intensiva}

Uma vez relacionadas e definidas, todas as formas de energia significativas em um ciclo motor, é possível representar a exergia total por meio da equação,

$$
E_{T}=\left(U-U_{0}\right)+p_{0}\left(V-V_{0}\right)-T_{0}\left(S-S_{0}\right)+\frac{m V^{2}}{2 g_{0}}+\frac{m g Z}{g_{0}}+E_{C H}
$$

A representação, em base molar ou mássica, da exergia total é dada pela equação, 


$$
e_{T}=\left(u-u_{0}\right)+p_{0}\left(v-v_{0}\right)-T_{0}\left(s-s_{0}\right)+\frac{V^{2}}{2 g_{0}}+\frac{g Z}{g_{0}}+e_{C H} .
$$

\section{$2.4 \quad$ O meio ambiente}

Considerando que o estado de equilíbrio com o meio ambiente é um dos referenciais para a definição de exergia, serão apresentadas algumas definições destinadas a complementar e tornar mais preciso o conceito de meio ambiente.

Conforme citado anteriormente, exergia é uma propriedade associada à interação entre o ambiente que se deseja estudar e o sistema. Análises baseadas na $1^{\mathrm{a}}$ Lei da Termodinâmica utilizam o conceito de sistema e vizinhança, onde o sistema representa o objeto de interesse e a vizinhança tudo aquilo que não pertence ao sistema. Assim, o ambiente deve ser definido como uma porção da vizinhança, com propriedades caracterizadas para o estudo da exergia. As principais características, do sistema assim considerado, são:

- O ambiente é um sistema, sendo, portanto, uma porção da vizinhança e não toda esta.

- O ambiente é composto de substâncias normalmente encontradas na atmosfera, litosfera e hidrosfera e estas substâncias devem estar em suas formas estáveis.

- O ambiente possui características termodinâmicas uniformes, ou seja, não há possibilidade de geração de trabalho através de interações, de qualquer natureza, de partes do mesmo.

- O ambiente possui suas propriedades intensivas uniformes (temperatura, pressão, volume específico, composição química).

- Propriedades extensivas do ambiente podem ser alteradas por interações com outros sistemas.

- As energia cinética e potencial do sistema devem ser consideradas tendo como referência o ambiente. Na grande maioria das aplicações o ambiente é considerado em repouso e possuindo energia potencial nula.

- Ao ambiente não está associada nenhuma forma de irreversibilidade, uma vez que não ocorrem, por hipótese, interações entre partes do mesmo. Dessa forma as irreversibilidades devem ocorrer apenas nos sistemas que interagem com o ambiente.

- Sendo assumidas as condições de repouso e energia potencial nula do ambiente, eventuais variações destas energias estarão relacionadas a causas ou fatores do próprio ambiente e não de suas interações com o sistema.

Obviamente o ambiente como descrito anteriormente é uma idealização e deve ser considerado somente para caracterizar a exergia associada ao sistema analisado. No contexto deste trabalho, cujo objetivo é a analise de sistemas para a geração de energia, o ambiente deverá ser considerado como uma porção da atmosfera ao redor do sistema analisado. Esta porção deverá ser grande o suficiente para que não haja alterações na sua temperatura e pressão em decorrência de interações com sistemas. Os valores de temperatura e pressão serão considerados como os valores médios de temperatura e pressão ambiente locais. Caso seja utilizada alguma fonte de água local, a temperatura do ambiente será a temperatura média desta fonte.

\subsection{Exergia para o sistema}

Neste ponto deseja-se analisar um sistema que passa por um processo entre um estado inicial 1 até um estado final 2. Como se trata de um sistema, não há transferência de massa. Para simplificar a análise, considera-se um processo que não altere os valores da exergia química associada ao sistema. Nestas condições, os balanços de energia e entropia são dados, respectivamente, por, 


$$
\left(U_{2}-U_{1}\right)+\left(\frac{m V_{2}^{2}}{2 g_{0}}-\frac{m V_{1}^{2}}{2 g_{0}}\right)+\left(\frac{m g Z_{2}}{g_{0}}-\frac{m g Z_{1}}{g_{0}}\right)=\int_{1}^{2} \delta Q-W
$$

$$
\left(S_{2}-S_{1}\right)=\int_{1}^{2}\left(\frac{\delta Q}{T}\right)_{f}+S_{g e n}
$$

Na equação (2.16) o subíndice $f$ indica a fronteira do sistema e a temperatura $T$ representa a temperatura na qual a transferência de calor $Q$ se processa.

A multiplicação da equação (2.16) pela temperatura ambiente $T_{0}$, muda sua dimensão de forma a permitir sua composição com a equação (2.15), que resulta na equação (2.17),

$$
\left(U_{2}-U_{1}\right)+\left(\frac{V_{2}^{2}}{2 g_{0}}-\frac{V_{1}^{2}}{2 g_{0}}\right)+\left(\frac{m g Z_{2}}{g_{0}}-\frac{m g Z_{1}}{g_{0}}\right)-T_{0}\left(S_{2}-S_{1}\right)=\int_{1}^{2} \delta Q-T_{0} \int_{1}^{2}\left(\frac{\delta Q}{T}\right)_{f}-W-T_{0} S_{g e n} .
$$

Considerando o valor da exergia total representada pela relação 2.13 , a equação 2.17 pode ser reorganizada de maneira a representar a variação de exergia de um processo entre os estados inicial 1 e final 2 sob a forma,

$$
E_{2}-E_{1}=\int_{1}^{2}\left(1-\frac{T_{0}}{T_{f}}\right) \delta Q-\left[W-p_{0}\left(V_{2}-V_{1}\right)\right]-T_{0} S_{g e n},
$$

onde os três termos do segundo membro da equação representam, respectivamente, a exergia associada aos fluxos de calor, toda a exergia associada às transferências de trabalho e à destruição de exergia devido às irreversibilidades internas.

\subsection{Exergia para o volume de controle}

A principal diferença entre as análises termodinâmicas de sistemas e de volumes de controle é que nas circunstâncias que exigem a análise por meio de volumes de controle, além das formas convencionais de energias de transição, existem fluxos de energia associados aos fluxos de massa que cruzam a superfície de controle. Para a representação da conservação da energia no volume de controle é adotada neste trabalho a formulação proposta por BEJAN et al (1996), sob a forma

$$
\frac{d E_{v c}}{d t}=\sum_{j}\left(1-\frac{T_{0}}{T_{j}}\right) \dot{Q}_{j}-\left(\dot{W}_{v c}-p_{0} \frac{d V_{v c}}{d t}\right)+\sum \dot{E}_{e}-\sum \dot{E}_{s}-\dot{E}_{D}
$$

onde os termos $\sum \dot{E}_{e}$ e $\sum \dot{E}_{s}$ representam os fluxos de energia associados aos fluxos de massa que entram e saem do volume de controle, respectivamente. O subíndice $v c$ representa o volume de controle e o subíndice $j$ refere-se à quantidade de fluxos de calor $\dot{Q}_{j}(j=1,2, \ldots)$ entre uma vizinhança com temperatura ambiente $T_{0}$ e o volume de controle.

Para calcular o valor da exergia associada aos fluxos de massa que atravessam o volume de controle é utilizada a metodologia proposta por MORAN \& SCIUBBA (1994), que considera a 
exergia associada aos fluxos de massa como sendo a exergia total original do fluxo de massa " $e$ " subtraída da energia transformada em trabalho de escoamento, representada pela relação,

$$
\dot{E}_{f}=\dot{m}\left[e-p_{0}\left(v_{0}-v\right)\right] \text {. }
$$

Pode-se observar que o trabalho de escoamento aqui explicitado se trata do mesmo trabalho definido anteriormente na equação (2.2) dividido pelo fluxo de massa.

Substituindo-se a equação (2.14) na equação (2.20) e dividindo o resultado pelo fluxo de massa, têm-se a equação para a exergia específica $e_{f}$ associada ao volume de controle

$$
e_{f}=\left(h-h_{0}\right)-T_{0}\left(s-s_{0}\right)+\frac{V^{2}}{2 g_{0}}+\frac{g Z}{g_{0}}+e_{C H} .
$$

Nesta equação, os termos $h$ e $h_{0}$ representam as entalpias do sistema e do ambiente respectivamente. Substituindo a primeira parte da equação (2.20) na equação (2.19) e assumindo a condição de regime permanente, têm-se então a equação para o balanço de exergia em um volume de controle sob a forma

$$
\dot{E}_{W}=\sum_{j}\left(1-\frac{T_{0}}{T_{j}}\right) \dot{Q}_{j}+\sum \dot{m}_{e} e_{e}-\sum \dot{m}_{s} e_{s}-\dot{E}_{D}
$$

Simplificando a equação (2.22), de forma a evidenciar as diferentes modalidades de exergia que compõem o processo analisado, é possível representá-la sob a forma,

$$
\dot{E}_{W}+\sum \dot{E}_{s}=\dot{E}_{Q}+\sum \dot{E}_{e}-\dot{E}_{D}
$$

Nesta equação, $\dot{E}_{W}$ representa a exergia associada aos fluxos de trabalho, $\dot{E}_{Q}$ representa a exergia associada aos fluxos de calor, $\dot{E}_{e}$ e $\dot{E}_{s}$ representam, respectivamente, a exergia associada aos fluxos de massa que entram e saem do volume de controle e $\dot{E}_{D}$ representa a exergia dissipada devido às irreversibilidades.

Considerando apenas fluxos de exergia que cruzam a superfície de controle, a equação de balanço assume a forma,

$$
\sum \dot{E}_{e}=\sum \dot{E}_{s}+\dot{E}_{D}
$$

Neste caso, considera-se agora que o termo $\dot{E}_{e}$ contabiliza a exergia associada aos fluxos de massa que entram e ao calor e o termo $\dot{E}_{s}$ representa a exergia associada aos fluxos de massa que saem e ao trabalho.

Pode-se observar que, para um volume de controle operando em regime permanente, a quantidade de exergia que deixa o volume de controle é sempre menor do que a admitida e a diferença entre estas está associada à destruição de exergia devido às irreversibilidades do processo. 
Uma vez definidas as equações que expressam o balanço de exergia em um volume de controle, têm-se as ferramentas necessárias para a análise de todos os componentes utilizados em sistemas térmicos destinados à geração de energia e que serão considerados neste trabalho, a menos da exergia química. Para o cálculo da exergia química, deve-se lançar mão de expressões adequadas para os casos a serem analisados neste trabalho.

\subsection{Exergia química}

Tratando-se especificamente de sistemas térmicos, são comuns situações onde o sistema analisado é composto unicamente por uma mistura de gases perfeitos e todos os componentes do sistema estão presentes no ambiente. Admitindo esta condição, pode-se adotar as equações propostas por MORAN \& SCIUBBA (1994), onde o potencial químico de cada substância nas condições do sistema, $\mu_{i}$, e do ambiente, $\mu_{i}$, é dado por,

$$
\begin{gathered}
\mu_{i}=\bar{g}_{i}\left(T_{0}, p_{0}\right)+\bar{R} T_{0} \ln \left(x_{i}\right), \\
\mu_{i 0}=\bar{g}_{i}\left(T_{0}, p_{0}\right)+\bar{R} T_{0} \ln \left(x_{i 0}\right) .
\end{gathered}
$$

Nestas equações $\bar{g}_{i}$ representa a função de Gibbs da substância analisada, em base molar, à temperatura $T_{i}$ e pressão $p_{i}$, onde $(i=0,1,2, \ldots)$. Neste caso, se o valor atribuído a $i$ for " 0 " (zero) as propriedades termodinâmicas, temperatura $T_{0}$ e pressão $p_{0}$ estarão relacionadas às condições do ambiente. A constante $\bar{R}$ é a constante universal para um gás ideal, $x_{i}$ é a fração molar da substância, $i$ e $x_{i 0}$ a fração correspondente para a condição do ambiente. Pode-se observar indutivamente que a função de Gibbs na equação (2.25) deveria corresponder à condição do sistema. Entretanto, o potencial químico de substâncias químicas é o mesmo tanto para as condições ambientais como outras condições do sistema, assim pode-se utilizar as propriedades associadas ao ambiente sem causar nenhum efeito prático nos resultados.

Substituindo as expressões (2.25) e (2.26) na equação (2.10) e dividindo o resultado pela quantidade de moles de mistura presente no sistema, têm-se a exergia química $e^{C H}$ para o sistema em base molar

$$
e^{C H}=\sum \bar{g}_{i}\left(T_{0}, p_{0}\right) x_{i}-\sum \bar{g}_{i}\left(T_{0}, p_{0}\right) x_{i 0}+\bar{R} T_{0} \sum_{i} x_{i} \ln \left(\frac{x_{i}}{x_{i 0}}\right)
$$

Considerando reações químicas que ocorrem reversível e isotermicamente, tam-se que a exergia correspondente é aproximadamente igual a variação da função de Gibbs durante a reação, tendo em vista que o $3^{\circ}$ membro do lado direito da equação (2.27) é muito menor do que os demais termos da mesma equação. No caso de reações que envolvem combustíveis constituídos por hidrocarbonetos, a diferença entre a variação da função de Gibbs e o poder calorífico inferior é muito pequena. Assim, como é prática usual, neste trabalho será considerado para a exergia química do combustível (Gás Natural - Metano) o seu poder calorífico inferior.

\subsection{Matéria prima, produtos e perdas}

Conforme citado anteriormente, o objetivo do trabalho será alcançado através da análise exergética de cada componente do sistema de geração de energia. Entretanto deve-se estabelecer um parâmetro de análise que seja aplicável a todos os componentes e que permita a adequação das peculiaridades associadas a cada um destes. Classificando convenientemente os fluxos de exergia que entram e saem do volume de controle, pode-se definir um parâmetro, tal como uma eficiência baseada somente nos fluxos de exergia. Ao se estabelecer tal parâmetro, dispõe-se de um critério de análise e assim pode-se mensurar quantitativamente que parcela de contribuição está associada a cada componente para o valor global da eficiência do sistema. 
Uma classificação muito útil e que atende plenamente as necessidades acima citadas, é a classificação dos fluxos de exergia como matéria prima, produtos e perdas. Essa classificação define claramente que forma de exergia está sendo considerada em cada componente, de acordo com a finalidade de cada um isoladamente. O balanço exergético decorrente da classificação de matéria prima, produtos e perdas é dado por,

$$
\dot{E}_{m}=\dot{E}_{p}+\dot{E}_{l}+\dot{E}_{d}
$$

Nesta equação, $\dot{E}_{m}, \dot{E}_{p}$ e $\dot{E}_{l}$ representam os fluxos de exergia que são classificados como matéria prima, produtos e perdas, respectivamente. $\mathrm{O}$ termo associado às perdas representa uma exergia que pode ser eventualmente reaproveitada. O último termo $\dot{E}_{d}$ representa a destruição de exergia devido às irreversibilidades que ocorrem no sistema.

\subsection{Eficiência exergética e eficiência exergética maior}

Neste ponto pode-se definir os conceitos de eficiência exergética " $\varepsilon$ " e eficiência exergética maior " $\varepsilon$ ". Utilizando as classificações de exergia anteriormente definidas, pode-se estabelecer eficiências inerentes a cada componente, que também podem ser estendidas à análise do sistema global. Essas eficiências são expressas por,

$$
\begin{gathered}
\varepsilon=\frac{\dot{E}_{p}}{\dot{E}_{m}}=1-\frac{\dot{E}_{l}+\dot{E}_{d}}{\dot{E}_{m}} . \\
\varepsilon^{+}=\frac{\dot{E}_{p}+\dot{E}_{l}}{\dot{E}_{m}}=1-\frac{\dot{E}_{d}}{\dot{E}_{m}} .
\end{gathered}
$$

Para o cálculo da eficiência do sistema $\varepsilon_{s i s}$, será utilizada a eficiência exergética definida pela relação,

$$
\varepsilon s i s=1-\frac{\dot{E}_{l}+\dot{E}_{d}}{\dot{E}_{m}} .
$$

Como a destruição de exergia de todo o sistema é dada pela soma da destruição de exergia em cada componente, a eficiência do sistema pode ser expressa pelo somatório da eficiência dos componentes,

$$
\varepsilon_{s i s}=1-\sum_{k}\left[\frac{\dot{E}^{k}}{\dot{E}_{m}}\left(1-\varepsilon_{k}^{+}\right)\right]-\frac{\dot{E}_{l}}{\dot{E}_{m}}
$$

Nesta equação, o índice $k$ representa o $k$-ésimo componente presente no sistema.

\section{Ciclo combinado Brayton - Rankine}

3.1 Sistemas de co-geração 
Co-geração é o processo de geração simultânea de energia elétrica e térmica, a partir de uma única fonte primária de energia. É um conceito técnico antigo que atualmente oferece um grande potencial de aplicação, em função da conjugação do progresso que se tem obtido nos mais diversos campos da técnica, da alta eficiência e da alta confiabilidade dos componentes utilizados.

No Brasil, em função da alta disponibilidade e do baixo custo da energia elétrica de base hidroelétrica fornecida pelas concessionárias, esta técnica tem uma aplicação modesta na matriz energética brasileira. Entretanto, com a tendência ao aumento das tarifas, à baixa disponibilidade de energia e ao aumento da oferta de gás natural, a utilização do processo de co-geração tende a ser econômica e estrategicamente vantajosa.

A co-geração tem um potencial de aplicação para indústrias que consomem vapor de forma intensiva em seu processo produtivo. Basicamente a relação entre energia elétrica e energia térmica, $\mathrm{E} / \mathrm{H}$, define a aplicabilidade de sistemas de co-geração para os diversos tipos de indústrias, com suas respectivas relações $\mathrm{E} / \mathrm{H}$ típicas ou determinadas caso a caso.

\subsubsection{Perspectivas para a co-geração}

As perspectivas de desenvolvimento da co-geração no Brasil são favoráveis, sobretudo com a recente reestruturação do setor elétrico, que já vem repercutindo na maior participação de investidores privados. Nesse aspecto, deve-se ressaltar que, para os empreendedores, a atividade de co-geração possui as seguintes vantagens estratégicas relativamente a projetos convencionais de geração:

- possibilita a redução nos custos devido à economia de combustíveis;

- tipicamente é um empreendimento de menor porte, que possibilita a utilização de equipamentos da indústria nacional, com custos reduzidos. Além disso proporciona uma maior atratividade a investimentos com prazos reduzidos de implantação;

- quando destinada a autoprodução, dispensa procedimentos burocráticos relacionados à comercialização de energia, acelerando o processo de implantação e reduzindo riscos de mercado;

- tendo impacto ambiental reduzido, tem o processo de licenciamento facilitado.

A geração distribuída ou geração descentralizada, por diversas vezes encontrou, como barreira ao seu desenvolvimento, as naturais implicações econômicas da pequena participação individual dos agentes do mercado e dos efeitos de escala nos custos de geração. A racionalidade energética e os custos evitados com a co-geração podem colocar a geração distribuída em situação de maior atratividade aos investidores, com conseqüentes benefícios ao sistema elétrico e, em ultima instância, à sociedade. Por outro lado, a possibilidade de auto-suprimento, ou fornecimento exclusivo de diversas utilidades, reduz os riscos de mercado.

\subsection{Ciclo Rankine}

No ciclo Rankine é utilizado o calor proveniente da queima de combustíveis para geração de vapor numa caldeira ou gerador de vapor. A energia térmica gerada pode ser utilizada para fornecer calor a algum processo e para geração de energia elétrica em um gerador elétrico acionado por uma turbina a vapor. $\mathrm{O}$ rendimento térmico máximo deste processo é de aproximadamente $30 \%$.

As figuras 3.1 e 3.2 a seguir ilustram o ciclo Rankine convencional e o ciclo Rankine com co-geração. 


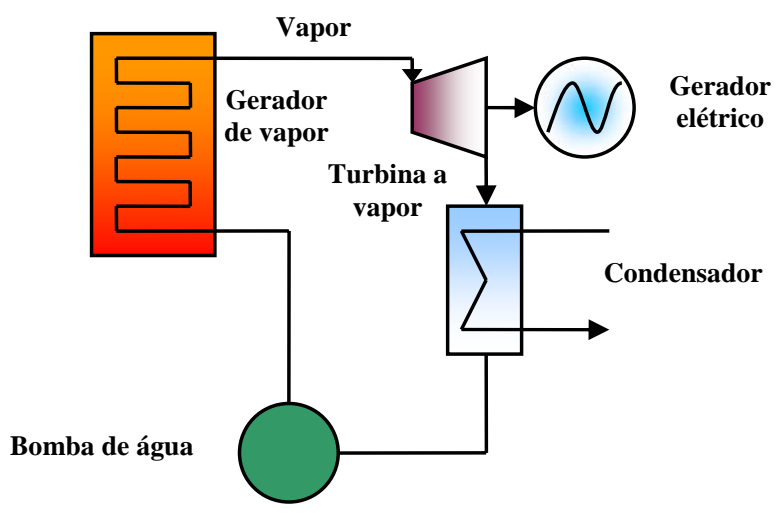

Figura 3.1 - Ciclo Rankine convencional

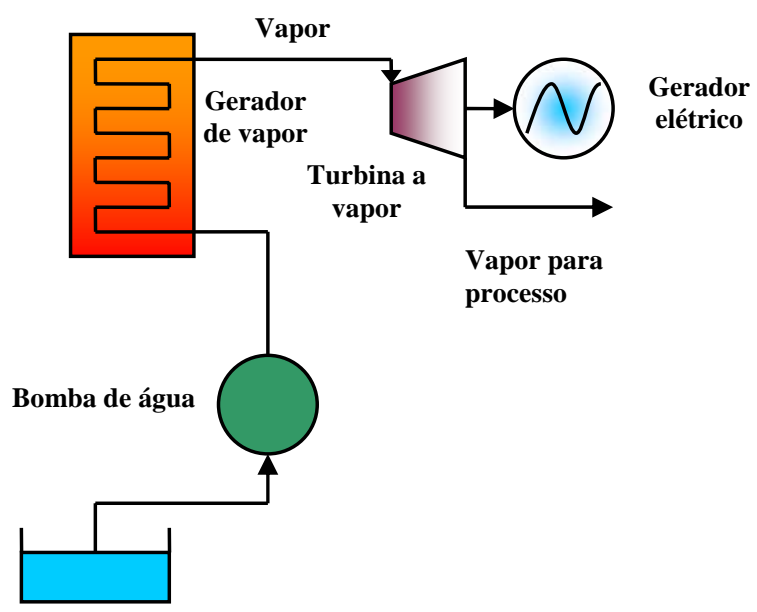

Figura 3.2 - Ciclo Rankine com co-geração

A co-geração, entretanto, utiliza o calor residual do vapor, geralmente de baixa pressão na exaustão da turbina de contra-pressão, ou de extração numa turbina de condensação. Isto é, o vapor é canalizado para ser aproveitado em seguida em processos de indústria ou aquecimento de água em trocadores de calor para posterior utilização. No caso das turbinas de contra-pressão parte do vapor que sai da turbina é desviada para a linha de processo enquanto na turbina de condensação, o calor é fornecido pelo processo de condensação do vapor após ser utilizado na turbina.

Este é o processo mais comumente utilizado em co-geração. Praticamente, todos os processos atuais de co-geração no Brasil o utilizam.

O rendimento térmico do ciclo com co-geração é calculado somando-se o total de energia utilizado, ou seja, a energia elétrica gerada mais a energia térmica utilizada no processo, dividindose pelo total da energia fornecida pelo combustível. A depender do processo, o rendimento térmico da co-geração pode chegar a $80 \%$.

\subsection{Ciclo Brayton}

O ciclo Brayton de turbina a gás vem se tornando um método cada vez mais utilizado para geração de energia. Neste tipo de máquina, ilustrada pela figura 3.3, o ar atmosférico é continuamente succionado pelo compressor, onde é comprimido até altas pressões. $\mathrm{O}$ ar comprimido entra na câmara de combustão, ou combustor, onde é misturado ao combustível criando condições para que ocorra a combustão, que tem como resultado uma violenta expansão dos gases devido às altas temperaturas atingidas durante o processo. Os gases provenientes da combustão se expandem através da turbina, gerando trabalho de eixo e são descarregados na atmosfera. Parte do trabalho desenvolvido pela turbina é usada para acionar o compressor, sendo o restante utilizado para acionar geradores elétrico ou dispositivos mecânicos diversos. 


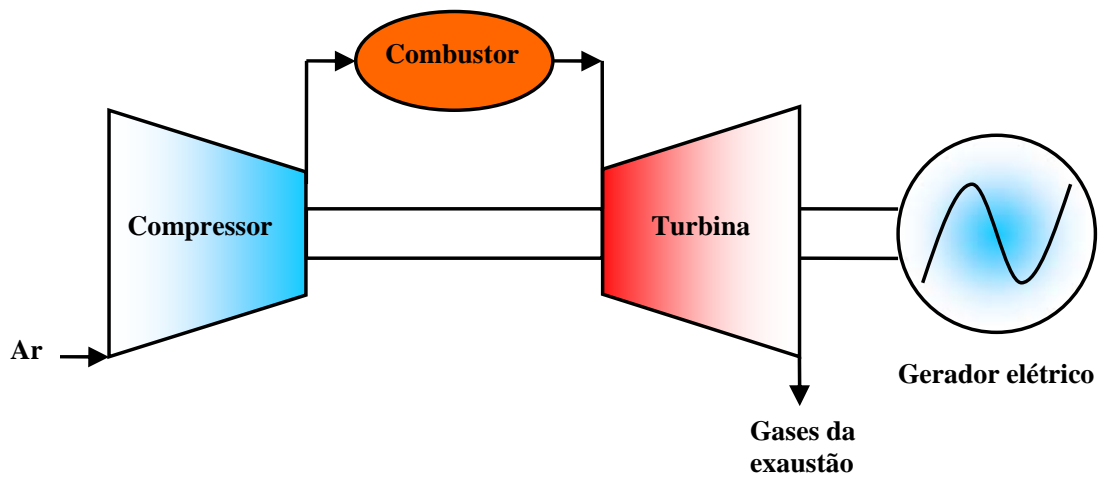

Fig 3.3 - Ciclo Brayton simples

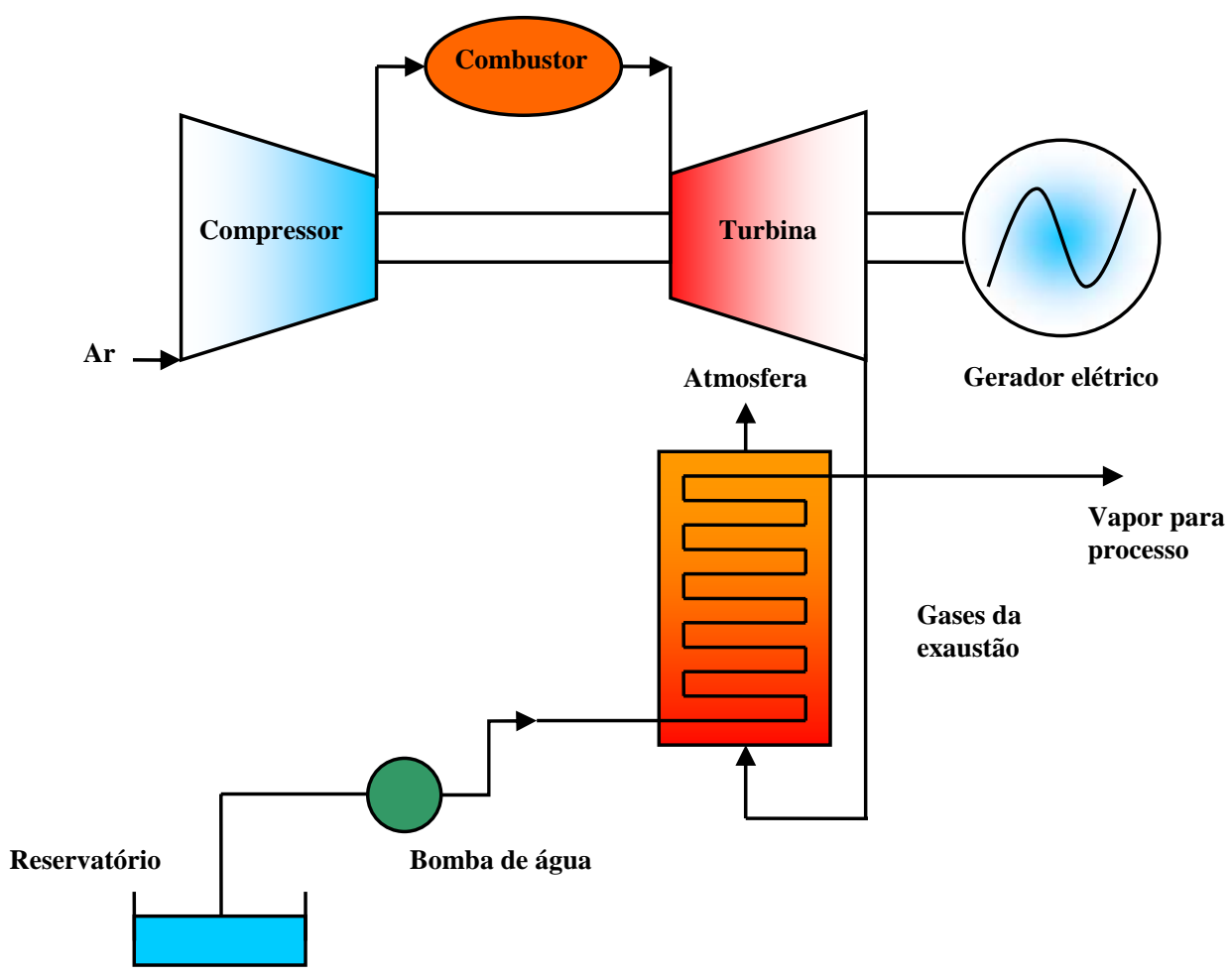

Fig 3.4 - Ciclo Brayton simples com co-geração

Este tipo de ciclo é chamado de ciclo Brayton simples e aberto, podendo existir algumas variações. O rendimento térmico do Ciclo Brayton é de aproximadamente $35 \%$, mas, atualmente, existem turbinas que atingem rendimentos de até $42 \%$. A co-geração neste ciclo é obtida através da adição, ao ciclo, de uma caldeira de recuperação de calor (figura 3.4). Neste caso os gases de exaustão da turbina são direcionados para uma caldeira, de modo a gerar vapor. Este vapor é então utilizado no processo industrial. Este é o ciclo proposto para a utilização do gás natural na maioria dos sistemas de co-geração atuais, utilizando como combustível o gás natural, que é a tendência atual no Brasil nas plantas nas quais o consumo de vapor é muito elevado, como nas indústrias de papel e celulose e químicas.

\subsection{Ciclo combinado Brayton - Rankine}

O ciclo combinado utiliza normalmente dois ciclos de potência, dentre os quais os mais freqüentes são os ciclos de Brayton e o de Rankine. A figura 3.5 ilustra um ciclo combinado deste tipo. Neste arranjo o calor liberado pelo ciclo de Brayton é usado parcial ou totalmente como entrada de calor para o ciclo de Rankine. No caso de geração de energia elétrica, através do ciclo combinado gás-vapor Brayton - Rankine, os gases de exaustão da turbina a gás estão numa 
temperatura relativamente alta, normalmente entre 450 e $550^{\circ} \mathrm{C}$, condição que permite seu emprego na geração de vapor que alimenta o ciclo de Rankine.

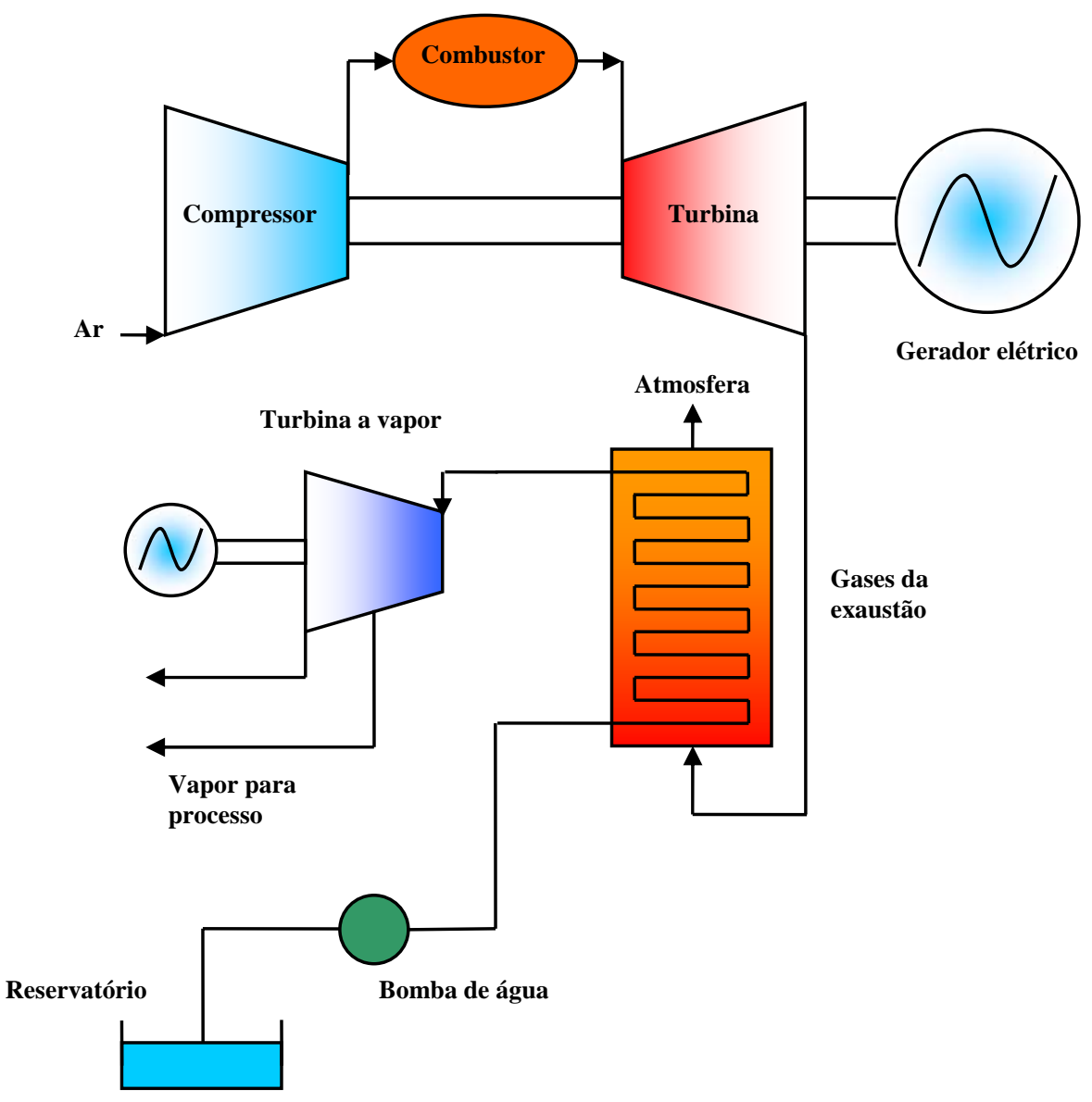

Fig 3.5 - Ciclo combinado com co-geração

Deste modo, o fluxo de gás quente pode ser utilizado numa caldeira de recuperação de calor para geração de vapor, que serve como fluido de trabalho para o acionamento de uma turbina a vapor, gerando um adicional de energia. Portanto, o ciclo combinado tem uma eficiência térmica maior que a dos ciclos Rankine e Brayton separadamente. Este tipo de processo de co-geração é a melhor opção para as aplicações nas quais a demanda de eletricidade é superior à demanda de vapor.

\subsection{Análise exergética do ciclo combinado}

A análise exergética do ciclo combinado Brayton-Rankine, deverá ser estruturada na análise isolada de cada componente do ciclo. Entretanto, deve-se definir quais serão os critérios de análise a serem utilizados em cada componente, pois cada um possui características bastante peculiares e tem uma finalidade específica dentro do sistema analisado. As características e peculiaridades de cada componente serão estudadas a seguir.

\subsection{Modelagem de componentes}

Os sistemas a serem modelados podem ser montados a partir de seus elementos básicos. Neste trabalho, cada um destes elementos será modelado segundo os pontos de vista termodinâmico e exergético. Os elementos a serem modelados podem ser identificados na figura 3.6 . 


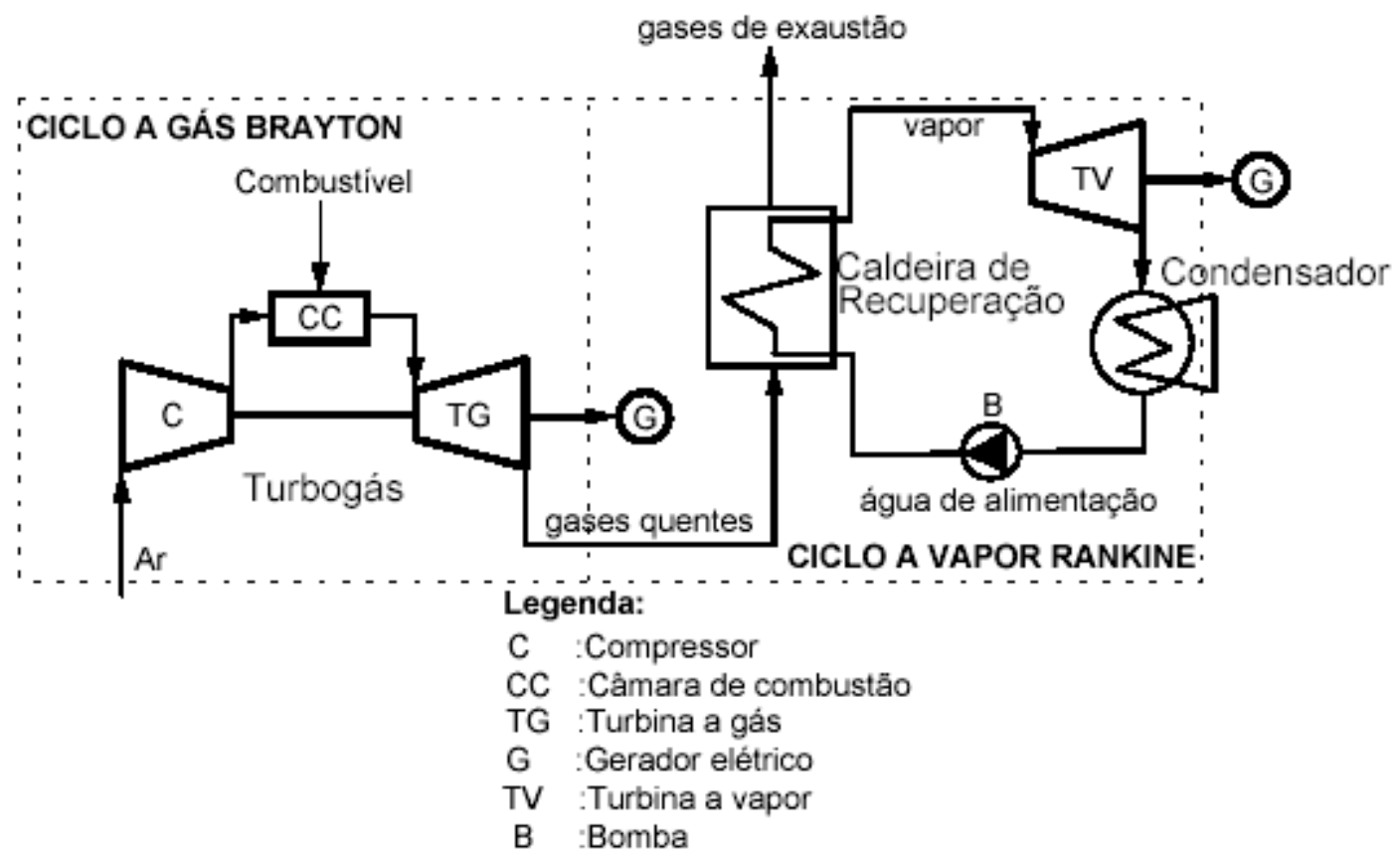

Figura 3.6 - Esquema de uma central a ciclo combinado gás/vapor

\subsubsection{Compressor}

A finalidade de um compressor é fornecer ar à alta pressão para que o mesmo seja utilizado no processo de combustão na turbina a gás e então seja expandido através da mesma. Desta forma, o compressor admite um fluxo de ar $\dot{n}_{a r}$, sob temperatura $T_{e}$ e pressão $p_{e}$ e recebe uma quantidade de trabalho $\dot{W}_{c p}$, fornecendo como produto ar a uma pressão $p_{s}$ e temperatura $T_{s}$.

Admitindo um fluxo em regime permanente, que é o regime normal de operação de todos os ciclos, tem-se que a vazão de admissão é necessariamente igual à vazão de descarga. Assim têmse que ambos os fluxos (entrada e saída) são iguais a $\dot{n}_{a r}$, (em base molar).

Considerando o ar como uma mistura de gases e observando que os processo de compressão não envolvem nenhuma reação química ou mistura de fluxos, tem-se que a composição do ar na entrada é igual à composição na saída.

Finalmente, admitindo um processo de compressão adiabática no compressor, pode-se expressar o balanço de energia por,

$$
\dot{W}_{c p}=\dot{n}_{a r}\left(\bar{h}_{e}-\bar{h}_{s}\right),
$$

onde $\bar{h}_{e}$ e $\bar{h}_{s}$ são, respectivamente, as entalpias na entrada e na saída do compressor.

Neste ponto, são introduzidos alguns parâmetros básicos necessários à análise de desempenho funcional de compressores.

\subsubsection{Razão de pressões}

A razão de pressões, $r_{c p}$, é o quociente entre a pressão do ar na descarga, $p_{s}$, e na admissão, $p_{e}$, do compressor. Assim, a razão de pressões é dada por,

$$
r_{c p}=\frac{p_{s}}{p_{e}} .
$$


Caso não seja disponibilizada a relação de pressão, pode-se recorrer a relação de pressão isoentrópica $R_{c p \text { iso }}$, que é a mesma relação anteriormente definida com a diferença de considerar que a pressão de saída seja atingida através de um processo isoentrópico. A vantagem de utilizar $R_{c p \text { iso }}$ está na relação que esta possui com o rendimento do ciclo Brayton

$$
\eta=1-\frac{1}{R_{\text {cpiso }} \frac{(k-1)}{k}},
$$

onde $\eta$ é o rendimento do ciclo Brayton e $k$ é a relação entre o calor específico a pressão e volume constantes do fluido de trabalho respectivamente.

\subsubsection{Eficiência isoentrópica}

Para a determinação da temperatura do ar na descarga do compressor usa-se o conceito de eficiência isoentrópica, $n_{\text {cpiso }}$, que compara o ganho de energia do fluido que ocorre no compressor com o ganho que ocorreria em um processo isoentrópico. A equação que expressa essa eficiência é dada por,

$$
n_{c p i s o}=\frac{\left(\bar{h}_{s i s o}-\bar{h}_{e}\right)}{\left(\bar{h}_{s}-\bar{h}_{e}\right)},
$$

onde $\bar{h}_{\text {siso }}$ representa a entalpia do ar na saída do compressor em uma situação ideal de compressão isoentrópica.

\subsubsection{Balanço de exergia}

O balanço de exergia no compressor é obtido pela aplicação da equação (2.22). Neste caso, não existem fluxos de calor e considera-se que os fluxos de massa entrando e saindo do compressor são iguais. Assim, o balanço de exergia é dado por,

$$
\dot{W}_{c p}+\dot{n}_{a r}\left(\bar{e}_{e}-\bar{e}_{s}\right)-\dot{E}_{d}=0
$$

onde $\dot{W}_{c p}$ é o trabalho aplicado sobre o compressor e $\dot{n}_{a r}$ a vazão mássica em base molar.

Pode-se agora definir os fluxos que entram e saem do compressor através da definição de matéria prima, produtos ou perdas. Assim tem-se que,

Matéria prima: fluxo de ar admitido e trabalho fornecido.

$$
\dot{E}_{m}=\dot{W}_{c p}+\dot{n}_{\text {ar }} \bar{e}_{e} .
$$

Produtos: fluxo de ar que deixa o compressor.

$$
\dot{E}_{p}=\dot{n}_{\text {ar }} \bar{e}_{s} .
$$


Perdas: não ocorrem perdas neste componente.

Agora pode-se definir uma expressão para a eficiência exergética do compressor. Essa expressão é dada por,

$$
\varepsilon_{c p}=\varepsilon_{c p}{ }^{+}=\frac{\dot{n}_{a r} \overline{\boldsymbol{e}}_{s}}{\dot{n}_{\text {ar }} \overline{\boldsymbol{e}}_{e}+\dot{W}_{c p}} .
$$

Como não ocorrem perdas os dois conceitos de eficiência, definidos pelas equações (2.29) e (2.30) são iguais.

\subsubsection{Combustor}

O combustor é o componente de maior importância em sistemas de turbinas a gás. Neste componente ocorre a queima do combustível que resulta na conversão de energia química do combustível em energia térmica. A este processo de conversão estão associadas as maiores irreversibilidades e portanto as maiores perdas presentes na instalação de geração de energia. Um esquema simplificado de um combustor é apresentado na figura 3.7 .

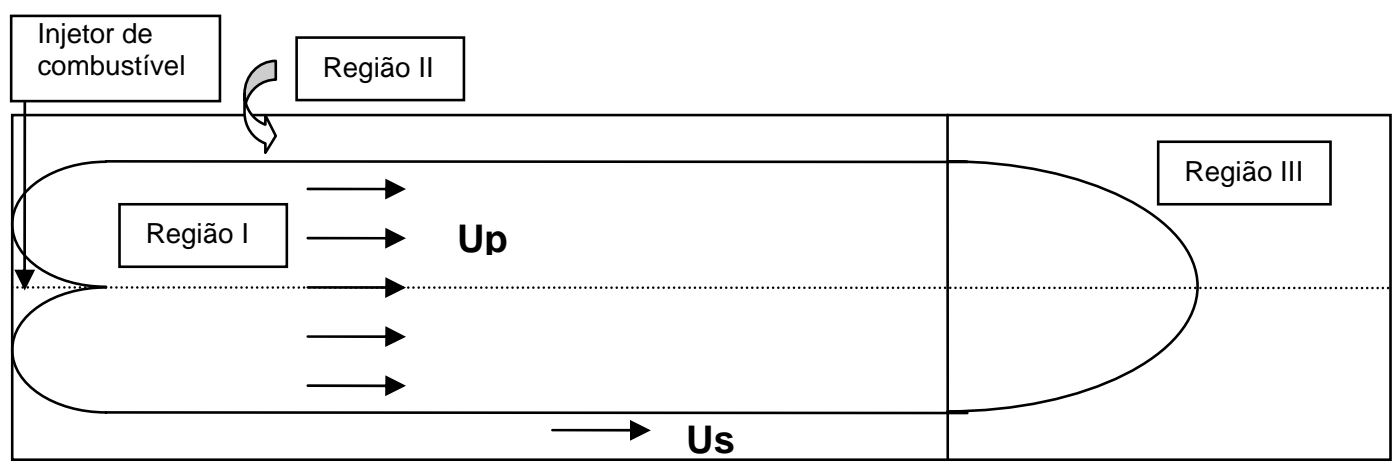

Figura 3.7 - Esquema simplificado do combustor

$\mathrm{Na}$ figura 3.7 mostra-se que o combustor pode ser dividido em três regiões distintas. A primeira, região I, é a zona de reação. Nesta região ocorre a mistura de ar com o combustível e todo o processo de combustão. A região II representa o escoamento secundário, que tem como uma de suas funções evitar o contato direto da chama com as paredes sólidas do combustor. O ar presente nesta região é continuamente transferido para a zona de reação de forma a alimentar o processo de queima. Na região III, zona de mistura, o fluxo de ar oriundo da região II mistura-se com os produtos da reação, oriundos da zona de reação. O resultado desta mistura é o produto final do combustor.

No contexto deste trabalho o combustor será modelado de acordo com o esquema da figura 3.8. Este esquema mostra que o ar admitido é utilizado em dois processos distintos. Uma parte é admitida em um reator, juntamente com o combustível onde ocorre o processo de combustão. A segunda parte mistura-se aos gases quentes gerados no reator na zona de mistura. Neste processo de mistura os gases são aquecidos até a temperatura na qual irão deixar o combustor. Desta forma existem três processos a serem modelados neste componente, a saber: a separação do ar admitido nas parcelas de queima e de resfriamento, o processo de combustão no reator e o processo de mistura dos gases quentes e do ar para resfriamento na zona de mistura. 


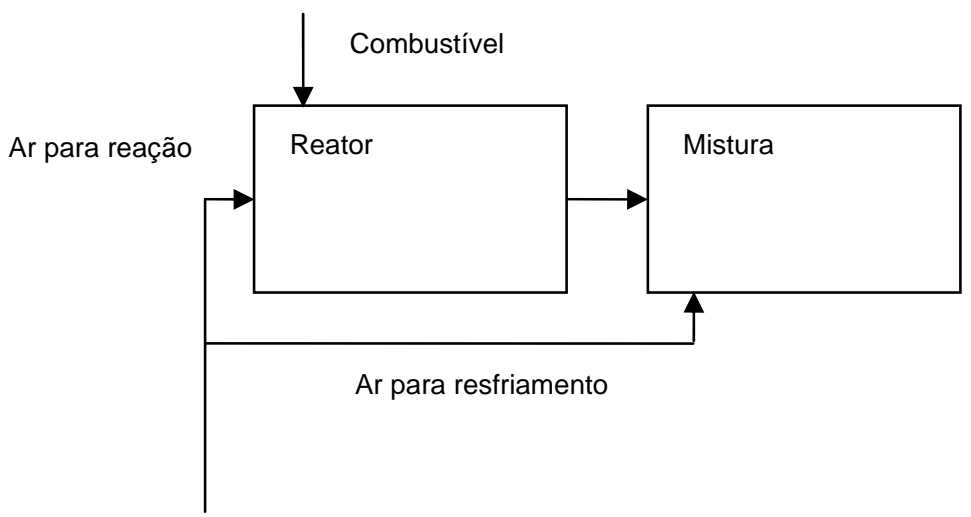

Figura 3.8 - Esquema do combustor - modelagem

\subsubsection{Separação do ar admitido}

Num dispositivo real onde um fluxo de massa seria dividido em dois haveria perdas que resultariam em queda da pressão e da temperatura das correntes de fluido. Neste modelo, entretanto, estas perdas não serão consideradas. Desta forma a temperatura e a pressão do ar para reação e para resfriamento igualam-se à temperatura e pressão do ar admitido. Esta igualdade é expressa pelas equações (3.9) a (3.12),

$$
\begin{aligned}
& T_{a d}-T_{a r}=0, \\
& T_{a d}-T_{a m}=0, \\
& P_{a d}-P_{a r}=0, \\
& P_{a d}-P_{a m}=0 .
\end{aligned}
$$

onde os sub-índices $a d$, $a r$ e am indicam, respectivamente, ar admitido, ar de reação e ar de mistura. O princípio de conservação da massa, para este processo, resulta na equação (3.13), onde $\dot{n}$ é a vazão molar e $M$ o peso molecular. Como neste processo não há alteração da composição química dos fluxos de massa, os pesos moleculares são iguais,

$$
\dot{n}_{a d M a d}-\left(\dot{n}_{a r M a r}+\dot{n}_{a m M a m}\right)=0
$$

Dessa forma, a equação (3.13) pode ser reescrita na forma abaixo,

$$
\dot{n}_{a d}-\left(\dot{n}_{a r}+\dot{n}_{a m}\right)=0 .
$$

A descrição completa deste processo exige ainda uma relação entre as vazões de ar para reação e resfriamento. Desta forma, define-se o parâmetro $\phi_{a r}$ como sendo a fração de ar destinada ao reator. Usando este parâmetro obtêm-se a equação (3.15),

$$
\dot{n}_{a d} \phi_{a r}-\dot{n}_{a r}=0 \text {. }
$$


O valor do parâmetro $\phi_{a r}$ é uma função de diversas características da câmara de combustão, dentre as quais destacam-se: materiais usados na construção e características do escoamento. Neste trabalho será considerado para $\phi_{a r}$, o valor 0.6, obtido a partir da análise dos trabalhos de COHEN et al (1996) e LEFEBVRE (1995).

3.6.2.2 Modelagem do processo de combustão

O objetivo da modelagem da combustão é obter as condições dos gases após este processo, ou seja, a determinação da pressão, temperatura e composição destes gases na saída. A pressão na descarga do reator é calculada considerando-se uma queda relativa fixa em relação à pressão de entrada do ar. Desta forma a pressão na descarga do reator é dada pela equação (3.16),

$$
p_{g r}-\left(1-\phi_{p c c}\right) p_{a d}=0
$$

onde o sub-índice gr indica gases do reator e $\phi_{p c c}$ é o coeficiente de perda de pressão no combustor. O valor de $\phi_{p c c}$ a ser utilizado, será o valor padrão de 0.05 proposto por BEJAN et al (1996).

A temperatura dos gases é obtida a partir de um balanço de energia no reator. Neste caso considera-se a energia associada aos fluxos de massa que cruzam a superfície de controle do combustor. Desta forma fazendo-se o balanço de energia obtêm-se a equação (3.17),

$$
\dot{n}_{a r} \bar{h}_{a r}+\dot{n}_{c b} \bar{h}_{c b}-\dot{n}_{g r} \bar{h}_{g r}=0
$$

onde $\bar{h}$ é a entalpia em base molar. Nesta equação os sub-índices $c b$ e $g r$ indicam, respectivamente, combustível e gases do reator. Deve-se notar que nesta equação o valor das entalpias é uma função da temperatura e da composição dos respectivos fluídos. O último dado a ser obtido na modelagem do reator é a composição dos gases. Esta será descrita a seguir.

\subsubsection{Modelo de combustão completa}

Neste caso o processo de queima de um combustível genérico na forma $C_{X} H_{Y}$ é descrito pela reação mostrada na equação (3.18), onde interpreta-se cada termo genérico $X_{i}^{j}$ como a fração molar $X$ de cada substância $i$ no ar e nos gases do reator em relação ao número de moles de combustível,

$$
\begin{gathered}
\dot{n}_{c b} \mathrm{CX}_{X} \mathrm{H}+\dot{n}_{a r}\left[X_{\mathrm{N}_{2}}^{a r} \mathrm{~N}_{2}+\mathrm{X}_{\mathrm{O}_{2}}^{a r} \mathrm{O}_{2}+\mathrm{X}_{\mathrm{CO}_{2}}^{a r} \mathrm{CO}_{2}+\mathrm{X}_{\mathrm{H}_{2} \mathrm{O}}^{a r} \mathrm{H}_{2} \mathrm{O}\right] \\
\Downarrow \\
\dot{n}_{g_{r}}\left[X_{\mathrm{N}_{2}}^{g r} \mathrm{~N}_{2}+X_{\mathrm{O}_{2}}^{g r} \mathrm{O}_{2}+X_{\mathrm{CO}_{2}}^{g r} \mathrm{CO}_{2}+X_{\mathrm{H}_{2} \mathrm{O}}^{g r} \mathrm{H}_{2} \mathrm{O}\right]
\end{gathered} .
$$

O balanço de espécies químicas na reação descrita pela equação (3.18) é dado, genericamente, pela equação (3.19), onde fij é, no combustível, o número de átomos do elemento $j$ na substância $i$ e $b k j$ é o número de átomos do elemento $j$ na substância $k$ no ar e nos gases de descarga.

Esta equação é escrita para os elementos carbono, hidrogênio, oxigênio e nitrogênio, donde obtêm-se um total de 4 equações que fornecem a composição dos gases na descarga do reator. 


$$
\dot{n_{c b}} \sum_{i} X_{i}^{c b} f_{i j}+\sum_{k} b_{k j}\left(\dot{n_{a r}} X_{k}^{a r}-\dot{n_{g r}} X_{k}^{g r}\right)=0
$$

Uma última equação faz-se, necessária para a determinação da vazão de gases gerados no reator. Para isso será considerado que o somatório das frações molares dos componentes presentes nos gases do reator deverão ser necessariamente iguais à unidade pois a referência para a fração molar será o número de moles de combustível fornecidos. Esse somatório é expresso pela equação (3.20),

$$
1-\sum_{i} X_{i}^{g r}=0
$$

Esta última equação fecha o modelo do reator para um caso de combustão completa. É válido ressaltar que este modelo de combustão não contempla a presença de outras substâncias que geralmente estão presentes nos produtos da combustão tais como monóxido de carbono $(C O)$ e óxidos de nitrogênio $\left(N O_{x}\right)$, para calcular as concentrações destas substâncias seria necessário um modelo mais sofisticado de reação química. Entretanto, como o objetivo principal do trabalho é a análise exergética, esta última observação será considerada somente para fins informativos.

\subsubsection{Mistura de gases e ar de resfriamento}

O processo de mistura, neste trabalho, é não reativo de forma que sua formulação é relativamente simples. As equações envolvidas serão (3.21) a (3.24). Nestas equações o índice g refere-se à condição dos gases deixando a zona de mistura e a câmara de combustão. A pressão final de descarga é considerada como a menor das pressões dos fluxos que entram na zona de mistura. Isto equivale a considerar que o gás na maior pressão expande-se até a pressão mais baixa. Esta formulação é representada pela equação (3.21),

$$
p_{g}-\min \left(p_{g r}, p_{a d}\right)=0 .
$$

A temperatura dos gases após a mistura é determinada pelo balanço de energia. Este balanço é representado pela equação (3.22),

$$
\dot{n}_{a m} \bar{h}_{a m}+\dot{n}_{g r} \bar{h}_{g r}-\dot{n}_{g} \bar{h}_{g}=0 .
$$

Por tratar-se de um processo não reativo o princípio de conservação de massa é escrito para cada substância de acordo com a equação (3.23), ou seja, o número de moles de cada substância se conserva no processo.

$$
\dot{n}_{a m} X_{i}^{a m}+\dot{n}_{g r} X_{i}^{g r}-\dot{n}_{g} X_{i}^{g}=0
$$

A última equação consiste em impor à composição final dos gases a condição de somatório igual à unidade. Ou seja, à composição total dos gases na saída é atribuído um valor unitário e a partir deste poderão ser comparadas as frações de cada substância na saída do combustor. Assim tem-se a equação (3.24), 


$$
1-\sum_{i} X_{i}^{g}=0
$$

Neste ponto encerra-se a modelagem do combustor.

\subsubsection{Balanço de exergia}

No caso do combustor poder-se-ia fazer uma análise detalhada para cada um dos processos que ocorrem no interior deste componente (separação, queima e mistura), todavia para fins de simplificação optou-se por uma análise simplificada que trata o combustor como um volume de controle único, figura 3.9. Neste caso é preciso que se considere o termo troca de calor. Todavia, utilizando-se o artifício de escolher um volume de controle no qual as perdas de calor ocorram à temperatura ambiente, o termo de perdas de exergia da equação (2.22) se anula. Assim, o balanço de exergia é expresso pela equação (3.25),

$$
\dot{n}_{\text {ar }} \bar{e}_{\text {are }}+\dot{n}_{c} \bar{e}_{c e}-\dot{n}_{g r} \bar{e}_{g r s}-\dot{E}_{D_{c c}}=0
$$

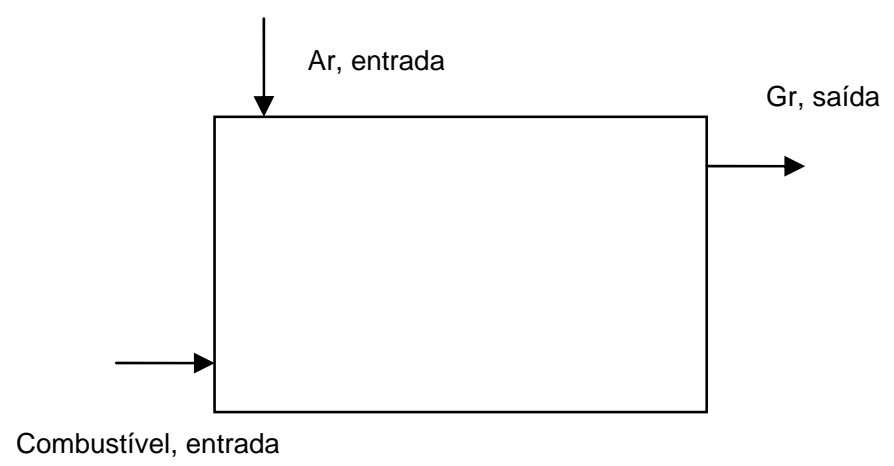

Figura 3.9 - Volume de controle para combustor - Análise exergética

Agora, considerando que a função do combustor é gerar gases a altas temperaturas utilizando combustível e ar, pode-se definir que fluxos de exergia são matéria-prima, produtos ou perdas. Assim tem-se,

Matéria prima: fluxos de massa admitidos no combustor.

$$
\dot{E}_{m}=\dot{n}_{\text {ar }} \overline{\boldsymbol{e}}_{\text {are }}+\dot{n}_{c} \bar{e}_{c e}
$$

Produtos: fluxo dos gases que deixam o combustor.

$$
\dot{E}_{p}=\dot{n}_{g r} \bar{e}_{\text {grs }} .
$$

Perdas: não ocorrem perdas neste componente.

Agora pode-se definir uma expressão para a eficiência exergética do combustor. Essa expressão é dada por,

$$
\varepsilon c c=\varepsilon_{c c}^{+}=\frac{\dot{n}_{g r} \bar{e}_{g r s}}{\dot{n}_{\text {ar }} \overline{\boldsymbol{e}}_{\text {are }}+\dot{n}_{c} \overline{\boldsymbol{e}}_{c e}}
$$




\subsubsection{Turbina a gás resfriada}

Um esquema da turbina resfriada é mostrado na figura 3.10. Neste modelo considera-se que os gases aquecidos e os de resfriamento misturam-se antes da entrada na turbina. A mistura é então expandida adiabaticamente até a pressão de descarga. Desta forma o modelo deste componente considera dois processos: o processo de mistura e o processo de expansão adiabática.

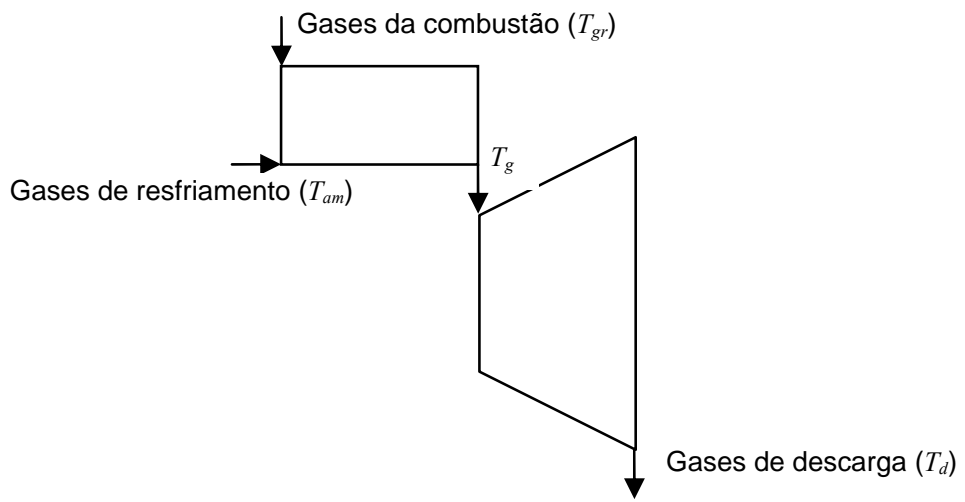

Figura 3.10 - Esquema de Turbina a gás

\subsubsection{Vazão de resfriamento}

A vazão de resfriamento já foi definida na modelagem do combustor é será calculada a partir do parâmetro $\phi_{a r}$ cujo valor foi anteriormente definido para 0.6. As equações a serem utilizadas para o balanço de massa serão,

$$
\begin{gathered}
\dot{n}_{a m}=\left(1-\phi_{a r}\right) \dot{n}_{a d}, \\
\dot{n}_{g}=\dot{n}_{a m+} \dot{n}_{g r} .
\end{gathered}
$$

\subsubsection{Processo de mistura}

O processo de mistura é baseado no princípio da continuidade que é representado pelas equações (3.31) e (3.32), onde se considera que este processo é não-reativo.

$$
\begin{gathered}
\dot{n}_{g r} X_{i}^{g r}+\dot{n}_{a m} X_{i}^{a m}-\dot{n}_{g} X_{i}^{g}=0, \\
1-\sum_{i} X_{i}^{g}=0 .
\end{gathered}
$$

A equação (3.31) deve ser escrita para cada uma das substâncias que compõem os gases do reator e a equação (3.32) estabelece que o somatório das frações molares de cada componente dos gases na saída do reator é igual à unidade.

A pressão dos gases após a mistura é dada pela equação (3.33)

$$
p_{g}-\min \left(p_{g r}, p_{a m}\right)=0 \text {. }
$$

O balanço de energia na zona de mistura permite o cálculo da temperatura dos gases após a mistura. Este balanço é expresso pela equação (3.34) 


$$
\dot{n}_{g r} \bar{h}_{g r}+\dot{n}_{a m} \bar{h}_{a m}-\dot{n}_{g} \bar{h}_{g}=0 .
$$

\subsubsection{Expansão adiabática}

Após a mistura os gases são expandidos de forma adiabática desde $\left(T_{g}, p_{g}\right)$ até a condição de descarga da turbina.

Durante a expansão não há mistura de fluxos, de forma que a composição e a vazão dos gases não se altera entre a entrada e a saída da turbina. Adotando-se essas considerações, o balanço de energia é expresso pela equação (3.35) onde se considera uma expansão adiabática

$$
\dot{W}_{c p}+\dot{W}_{l v}-\dot{n}_{g}\left(\bar{h}_{e g}-\bar{h}_{s g}\right)=0 .
$$

Além da equação da energia, a modelagem deste componente exige ainda a utilização do conceito de eficiência isoentrópica, definido pela equação (3.36). A seguir faz-se o uso do conceito de rendimento isoentrópico para determinar a temperatura na descarga da turbina, onde o termo "Stg iso representa um processo isoentrópico na turbina a gás entre a entrada e a saída da mesma

$$
\eta_{t g i s o}\left(\bar{h}_{s i s o}-\bar{h}_{e_{g}}\right)-\left(\bar{h}_{s g}-\bar{h}_{e_{g}}\right)=0 .
$$

A eficiência isoentrópica necessita de uma relação adicional que define a condição de saída para um processo de expansão isoentrópica. Esta condição é determinada pela equação (3.37)

$$
S_{\text {siso }}=S e g \text {. }
$$

\subsubsection{Balanço de exergia}

A modelagem exergética da turbina é expressa pela equação (3.38) obtida a partir de um balanço de exergia em um volume de controle envolvendo este componente

$$
\left(\dot{n}_{s_{g r}} \bar{e}_{s g r}+\dot{n}_{s a m} \bar{e}_{s a n}\right)-\left[\left(\dot{W}_{c p}+\dot{W}_{l v}\right)+\dot{n}_{s g} \bar{e}_{s g}\right]-\dot{E}_{D_{t g}}=0 .
$$

Agora, considerando que a função da turbina é gerar trabalho pode-se interpretar os fluxos de exergia como matéria-prima, produtos ou perdas. Assim tem-se

Matéria-prima: fluxo de gases admitidos

$$
\dot{E}_{m}=\dot{n}_{g r} \bar{e}_{s g r}+\dot{n}_{\text {am }} \bar{e}_{\text {sam }} .
$$

Produto: o fluxo de trabalho gerado

$$
\dot{E}_{p}=\dot{W}_{c p}+\dot{W}_{l v} .
$$

Perdas: gases de descarga 


$$
\dot{E}_{l}=\dot{n}_{g} \bar{e}_{s_{g}} .
$$

Agora pode-se utilizar a definição de eficiência exergética e eficiência exergética maior da turbina através das equações (3.42) e (3.43)

$$
\begin{gathered}
\varepsilon_{t g}=\frac{\dot{W}_{c p}+\dot{W}_{l v}}{\dot{n}_{g r} \bar{e}_{s g r}+\dot{n}_{a m} \bar{e}_{s a m}}, \\
\varepsilon_{t g}{ }^{+}=\frac{\dot{W}_{c p}+\dot{W}_{l v}+\dot{n}_{g} \bar{e}_{s g}}{\dot{n}_{g r}-\dot{e}_{s g r}+\dot{n}_{a m} \bar{e}_{s a m}}
\end{gathered}
$$

\subsubsection{Caldeira de recuperação de calor}

A função básica deste componente é aproveitar o conteúdo energético de um fluxo de gases a alta temperatura para gerar vapor. Assim, este componente recebe uma vazão de líquido a uma pressão especificada e deve aquecer este líquido de forma a obter vapor superaquecido.

Dentro deste componente o líquido recebido sofre três processos, a saber:

- É aquecido da temperatura de entrada até a temperatura de saturação de uma pressão especificada;

- Após ter atingido a condição de saturação, este líquido é vaporizado;

- O vapor gerado é superaquecido até uma temperatura especificada.

Desta forma este componente será modelado como um conjunto de três trocadores de calor operando em série, como indicado na figura 3.11.

Para a caldeira de recuperação serão definidos os seguintes parâmetros:

$\phi_{p x x}$---- coeficiente de queda de pressão no lado do vapor. O subíndice " $x x$ " pode ser substituído por $s a$ (superaquecedor), ev (evaporador) e $e c$ (economizador).

$\phi_{p g}$---- coeficiente de perda de pressão no lado dos gases. Em cada trocador interno.

$\phi_{q r c}$---- coeficiente de perda de calor.

$\dot{n}_{v s p}---$ vazão de vapor no superaquecedor.

$n_{\text {vec }}$---- vazão de vapor no economizador.

$\dot{n}_{\text {vext }}$--- vazão de vapor extraído na saída do economizador onde $\dot{n}_{\text {vext }}=\dot{n}_{\text {vec }}-\dot{n}_{v s p}$.

Além disto na figura 3.11 estão indicados os pontos de 1 a 4 que correspondem a:

1 ------- Entrada do economizador. Neste ponto os gases deixam a caldeira e o líquido é admitido. Também neste ponto os gases e o vapor estão em suas temperaturas mais baixas.

2 ------- Entrada do evaporador. Neste ponto pode-se fazer a extração de líquido $\dot{n}_{\text {vext }}$.

3 ------- Entrada do superaquecedor.

4 ------- Saída do superaquecedor. Neste ponto os gases e o vapor estão em suas temperaturas mais altas. 
Agora pode-se fazer a modelagem do componente.

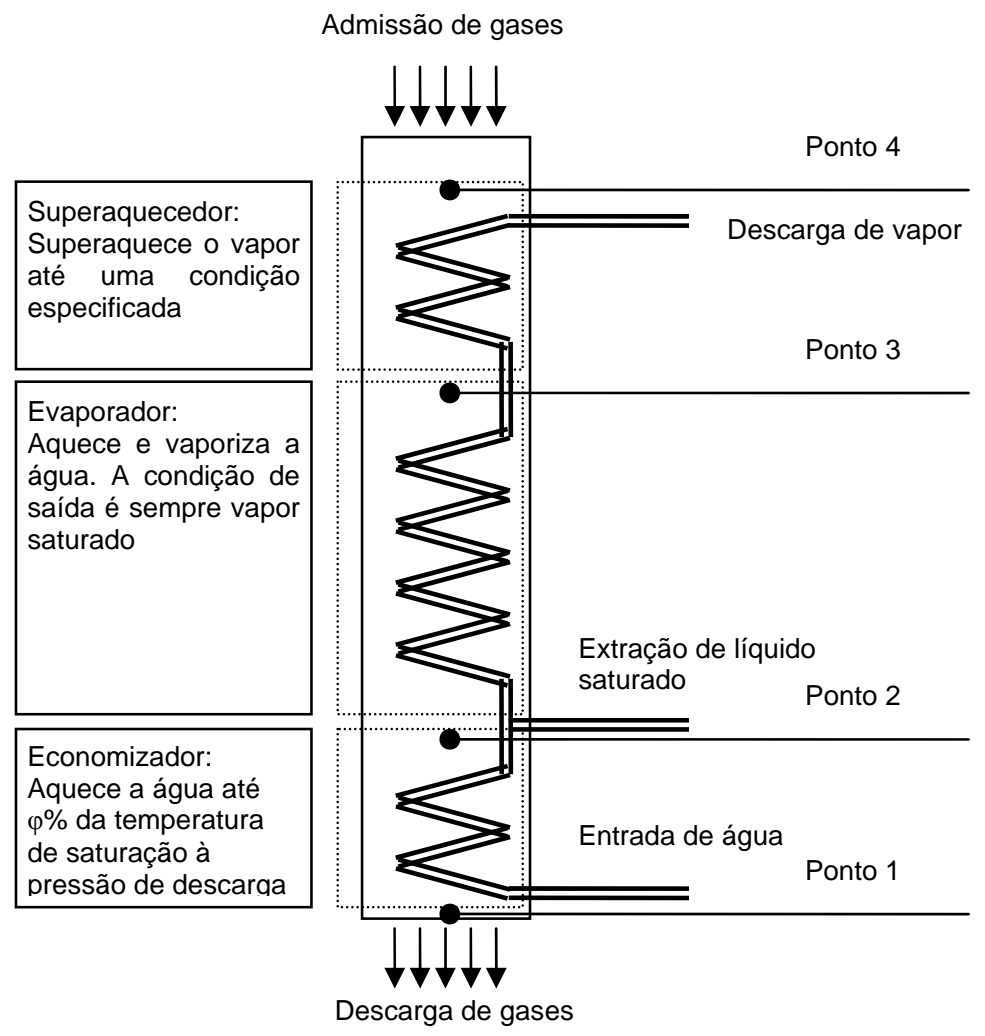

Figura 3.11 - Esquema de caldeira de recuperação de calor

\subsubsection{Balanço de massa}

Neste componente não existem processos reativos ou mistura de fluxos, dessa forma a composição dos fluidos não se altera. $\mathrm{O}$ balanço de massa pelo lado dos gases é dado pela equação (3.44)

$$
\dot{n}_{e_{g}}=\dot{n}_{s g}=\dot{n}_{g} .
$$

No lado do vapor deve-se considerar a possibilidade de extração de vapor no ponto 2 . Assim o balanço de massa é expresso pela equação (3.45). Note que devido às características da caldeira de recuperação a vazão de vapor no economizador é, necessariamente, igual à vazão no superaquecedor, uma vez que não existe a possibilidade de extração além do ponto 2

$$
\dot{n}_{v e c}=\dot{n}_{v e x t}+\dot{n}_{v s p} .
$$

\subsubsection{Balanço de energia}

Neste caso faz-se um balanço único para todo o componente. Este balanço é expresso pela equação (3.46). Segundo a metodologia proposta por CONSONNI \& LARSON (1996) o coeficiente de perda de calor neste componente é dado pela equação (3.47)

$$
\left(1-\phi_{q_{r c}}\right) \dot{n}_{g}\left(\bar{h}_{4_{g}}-\bar{h}_{1_{g}}\right)-\left(\dot{n}_{v_{s p}} \bar{h}_{4_{v}}+\dot{n}_{v_{v x t}} \bar{h}_{2_{v}}-\dot{n}_{v_{v e}} \bar{h}_{1_{v}}\right)=0
$$




$$
\phi_{q r c}=\left\{\begin{array}{ll}
0 & \text { se } T_{e_{g}}<573 K \\
0.02 & \text { se } T_{e_{g}} \geq 573 K
\end{array}\right\} .
$$

\subsubsection{Queda de pressão}

Ambos os fluidos estão sujeitos a queda de pressão durante a passagem pela caldeira de recuperação. No lado dos gases esta queda de pressão é dada pela equação (3.48) e no caso do vapor pela equação (3.49)

$$
\begin{aligned}
& p_{1 g}=\left(1-\phi_{p g}\right)^{3} p_{4_{g}}, \\
& p_{4_{v}}=\left(1-\phi_{p e c}\right)\left(1-\phi_{p e x t}\right)\left(1-\phi_{p s p}\right) p_{1_{v}} .
\end{aligned}
$$

\subsubsection{Efetividade}

A caldeira de recuperação é um trocador de calor composto por três trocadores de calor. Assim podem ser calculadas quatro efetividades para a caldeira. Neste ponto faz-se apenas o cálculo para todo o componente. Deve-se então observar todos os processos presentes na caldeira para definir sua efetividade. Os perfis de temperatura dentro da caldeira são mostrados na figura 3.12 .

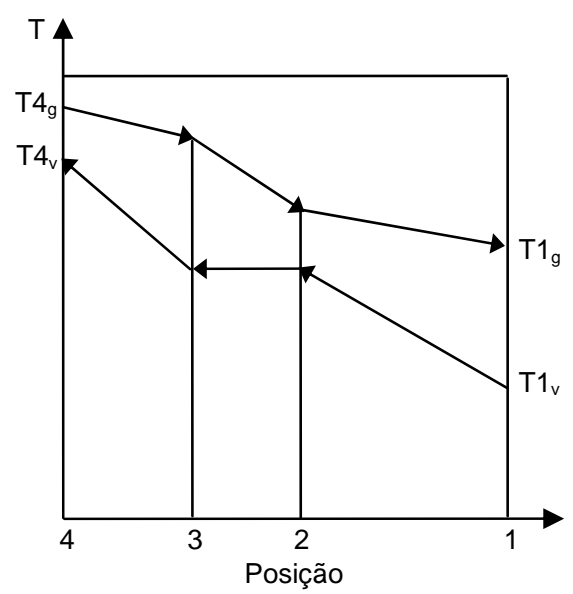

Figura 3.12 - Perfil de temperaturas na caldeira de recuperação de calor

A efetividade de todo trocador é então dada pela equação (3.50), onde $\bar{h}_{1, v}$ é a entalpia dos gases de descarga avaliada na temperatura de admissão do vapor

$$
e f_{r c}=\frac{\bar{h}_{4_{g}}-\bar{h}_{1 g}}{\bar{h}_{4_{g}}-\bar{h}_{1_{g, v}}} .
$$

\subsubsection{Balanço de exergia}

Nesta etapa faz-se a análise da caldeira de recuperação de forma global. A caldeira será considerada como sendo um trocador de calor onde o calor é transferido de um fluido quente (gases aquecidos) e um fluido frio (vapor). O balanço deste componente é dado pala equação (3.51) 


$$
\left(\dot{n}_{g} \bar{e}_{4_{g}}+\dot{n}_{e c}-\bar{e}_{1_{v}}\right)-\left(\dot{n}_{s p e_{4_{v}}}+\dot{n}_{\text {exte }} \bar{e}_{2_{v}}+\dot{n}_{g} \bar{e}_{1_{g}}\right)-\dot{E}_{D r c}=0 .
$$

Agora, considerando-se que a função da caldeira é gerar vapor, pode-se definir que fluxos de exergia são matéria-prima, produtos ou perdas. Assim tem-se:

Matéria-prima: fluxo de gases e líquido admitido

$$
\dot{E}_{m}=\dot{n}_{g} \bar{e}_{4_{g}}+\dot{n}_{e c} \bar{e}_{1_{v}} .
$$

Produtos: o fluxo de vapor gerado e o fluxo de líquido saturado gerado

$$
\dot{E}_{p}=\dot{n}_{s p} \bar{e}_{4_{v}}+\dot{n}_{\text {ext }} e_{2 v} .
$$

Perdas: gases de descarga

$$
\dot{E}_{l}=\dot{n}_{g} \bar{e}_{l_{g}} .
$$

A partir destes conceitos pode-se estabelecer as eficiências exergéticas deste componente, elas são dadas pelas equações (3.55) e (3.56)

$$
\begin{aligned}
& \varepsilon_{r c}=\frac{\dot{n}_{s p} \bar{e}_{4_{v}}+\dot{n}_{e x t} \bar{e}_{2_{v}}}{\dot{n}_{g} \bar{e}_{4_{g}}+\dot{n}_{e c} \bar{e}_{1_{v}}}, \\
& \operatorname{src}^{+}=\frac{\dot{n}_{s p} \bar{e}_{4_{v}}+\dot{n}_{\text {ext }}-\bar{e}_{2_{v}}+\dot{n}_{g} \bar{e}_{1_{g}}}{\dot{n}_{g} \bar{e}_{4 g}+\dot{n}_{e c}-\bar{e}_{v}} .
\end{aligned}
$$

\subsubsection{Turbina a vapor}

O esquema básico de uma turbina a vapor é mostrado na figura 3.13. a função básica deste componente é gerar trabalho a partir da expansão do vapor superaquecido.

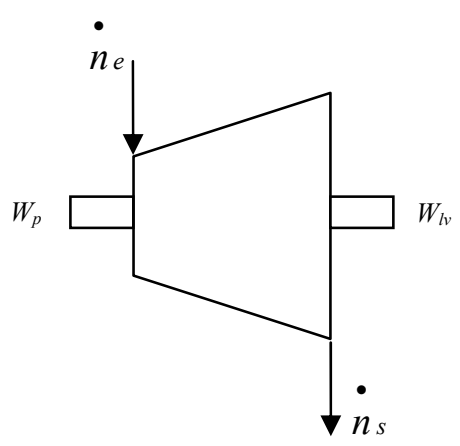

Figura 3.13 - Esquema de turbina vapor

3.6.5.1 Balanço de energia 
Considerando a turbina como sendo adiabática e aplicando o princípio da continuidade, o balanço de energia para este componente é expresso pela equação (3.57)

$$
\dot{W}_{p}+\dot{W}_{l v}=\dot{n}_{v}\left(\bar{h}_{e}-\bar{h}_{s}\right) .
$$

\subsubsection{Eficiência isoentrópica}

A seguir faz-se uso do conceito de rendimento isoentrópico para determinar a condição descarga da turbina. Este conceito é expresso pela equação (3.58) e a condição de saída para o processo isoentrópico é especificada pela equação (3.59)

$$
\begin{gathered}
\eta_{t v i s o}=\frac{\left(\bar{h}_{s}-\bar{h}_{e}\right)}{\left(\bar{h}_{\text {siso }}-\bar{h}_{e}\right)}, \\
S_{\text {Siso }}=S e .
\end{gathered}
$$

\subsubsection{Condição de descarga}

No caso da turbina a vapor existem duas possíveis condições de descarga: vapor superaquecido ou mistura de líquido e vapor (saturação). No primeiro caso a condição de descarga é determinada pela pressão e temperatura do vapor, enquanto no segundo caso a condição é determinada pela pressão e pelo título.

\subsubsection{Balanço de exergia}

A modelagem exergética da turbina a vapor é expressa pela equação (3.60) obtida a partir de um balanço de exergia em um volume de controle envolvendo este componente

$$
\left(\dot{W}_{p}+\dot{W}_{l v}\right)+\dot{n}_{v}\left(\bar{e}_{s}-\bar{e}_{e}\right)-\dot{E}_{D_{t v}}=0 .
$$

Considerando que a função da turbina a vapor é gerar trabalho, pode-se considerar que os fluxos de exergia são matéria-prima, produtos ou perdas. Assim tem-se:

Matéria-prima: fluxo de vapor admitido

$$
\dot{E}_{m}=\dot{n}_{v} \bar{e}_{e}
$$

Produtos: o fluxo de trabalho gerado

$$
\dot{E}_{p}=\dot{W}_{p}+\dot{W}_{l v} .
$$

Perdas: vapor descarregado

$$
\dot{E}_{l}=\dot{n}_{v} \bar{e}_{s} .
$$


Agora pode-se definir as eficiências exergéticas da turbina a vapor. Essas são expressas pelas equações (3.64) e (3.65)

$$
\begin{gathered}
\mathcal{E}_{v}=\frac{\dot{W}_{p}+\dot{W}_{l v}}{\dot{n}_{v} \bar{e}_{e}}, \\
\varepsilon_{t v}{ }^{+}=\frac{\dot{W}_{p}+\dot{W}_{l v}+\dot{n}_{v} \bar{e}_{s}}{\dot{n}_{v} \bar{e}_{e}} .
\end{gathered}
$$

\subsubsection{Trocador de calor}

Neste trabalho o trocador de calor a ser modelado será do tipo fluxo cruzado. O esquema para este tipo de trocador é mostrado na figura 3.14. Nesta figura nota-se os índices $f$ para fluido frio e $q$ para fluido quente. A função do trocador, no contexto deste trabalho, será sempre aquecer o fluido frio.

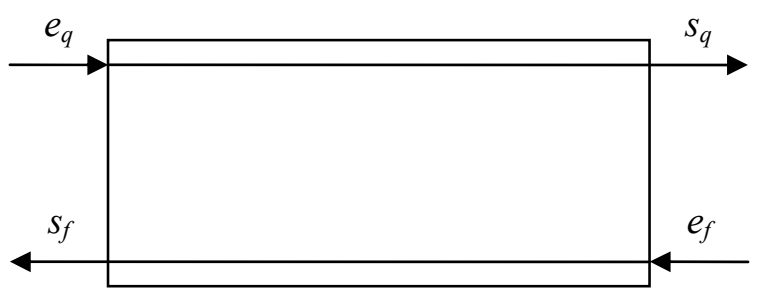

Figura 3.14 - Esquema de trocador de calor

\subsubsection{Balanço de massa}

No caso do trocador de calor não há mistura de fluxos de massa envolvidos. Assim o princípio da continuidade é expresso pelas equações (3.66) e (3.67)

$$
\begin{aligned}
& \dot{n}_{s q}=\dot{n}_{e q}=\dot{n}_{q}, \\
& \dot{n}_{s f}=\dot{n}_{e f}=\dot{n}_{f} .
\end{aligned}
$$

Neste componente não existem reações químicas ou misturas de fluxos, de forma que a composição dos fluidos não se altera.

\subsubsection{Balanço de energia}

O balanço de energia para o trocador de calor é expresso pela equação (3.68)

$$
\dot{n}_{q}\left(\bar{h}_{s q}-\bar{h}_{e q}\right)-\left[\dot{n}_{f}\left(\bar{h}_{s f}-\bar{h}_{e f}\right)+\dot{Q}_{t c r}\right]=0 .
$$

As perdas de calor, termo $\dot{Q}_{t c r}$, serão quantificadas como uma parcela fixa do calor total extraído do fluido quente, segundo a metodologia proposta por CONSONNI LARSON (1996) a fração de perda é dada pela equação (3.69) 


$$
\phi_{q c r}=\left\{\begin{array}{lll}
0 & \text { se } & T_{e_{q}}<573 K \\
0.02 & \text { se } & T_{e_{q}} \geq 573 K
\end{array}\right\} .
$$

Assim, o balanço de energia no trocador de calor passa a ser expresso pela equação (3.70)

$$
\left(1-\phi_{q t c r}\right) \dot{n}_{q}\left(\bar{h}_{s q}-\bar{h}_{e q}\right)-\dot{n}_{f}\left(\bar{h}_{s f}-\bar{h}_{e f}\right)=0 .
$$

\subsubsection{Queda de pressão estática}

Para a determinação das pressões usam-se coeficientes de perda para ambos os fluidos. As equações (3.71) e (3.72) representam a queda de pressão nos fluidos frio e quente

$$
\begin{aligned}
& p_{e f}\left(1-\phi_{p f}\right)-p_{s f}=0, \\
& p_{e q}\left(1-\phi_{p q}\right)-p_{s q}=0 .
\end{aligned}
$$

O valor dos coeficientes é 0.03 para o fluido quente e 0.05 para o fluido frio, segundo BEJAN et al (1996).

\subsubsection{Efetividade}

$\mathrm{Na}$ figura 3.15 tem-se o comportamento da temperatura dos fluidos no interior do trocador de calor, supondo que este seja um trocador do tipo fluxo cruzado.

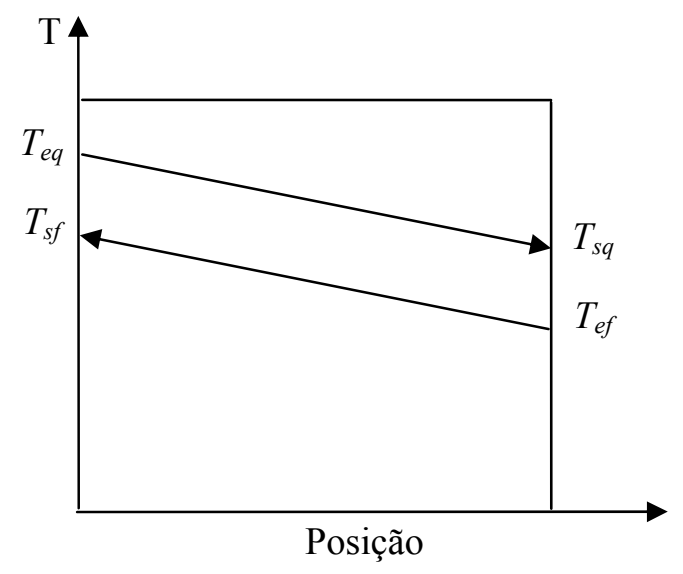

Figura 3.15 - Temperatura dos fluidos no trocador de calor

A efetividade é definida, supondo que a função do trocador seja aquecer o fluido frio, como a razão entre o calor efetivamente retirado do fluido quente no trocador real e o calor retirado caso o trocador tivesse uma área de troca infinita, ou seja os pontos $T_{s q}$ e $T_{e f}$ fossem coincidentes na figura 3.15. Desta forma, o conceito de efetividade é expresso pela equação (3.73)

$$
e f_{t c r}\left(\bar{h}_{e q}-\bar{h}_{s f, q}\right)-\left(\bar{h}_{e q}-\bar{h}_{s q}\right)=0 .
$$

Nesta equação, $\bar{h}_{s f, q}$ é a entalpia do fluido quente avaliada na temperatura de entrada do fluido frio. 


\subsubsection{Balanço de exergia}

O balanço de exergia no trocador é obtido pela aplicação da equação (2.22), sendo que neste caso é preciso que se considere o termo de troca de calor. Todavia utilizando-se o artifício de escolher um volume de controle no qual as perdas de calor ocorram à temperatura ambiente, o termo de perdas de exergia, presente na equação (2.22), anula-se. Assim o balanço de exergia no trocador de calor é expresso pela equação (3.74)

$$
\dot{n}_{f}\left(\bar{e}_{e f}-\bar{e}_{s f}\right)+\dot{n}_{q}\left(\bar{e}_{e q}-\bar{e}_{s q}\right)-\dot{E}_{D_{t c r}}=0 .
$$

Agora considerando que a função do trocador é aquecer o fluido frio pode-se considerar que fluxos de exergia são matéria-prima, produtos ou perdas. Assim tem-se:

Matéria-prima: fluxos de massa admitidos no trocador de calor

$$
\dot{E}_{m}=\dot{n}_{q} \bar{e}_{e_{q}}+\dot{n}_{f} \bar{e}_{e f} .
$$

Produtos: o fluxo de exergia associado ao fluido frio que é aquecido e deixa o trocador de calor

$$
\dot{E}_{p}=\dot{n}_{f} \bar{e}_{s f} .
$$

Perdas: o fluxo de exergia associado ao fluido quente que é resfriado e deixa o trocador de calor

$$
\dot{E}_{l}=\dot{n}_{q} \bar{e}_{s q} .
$$

Agora pode-se definir as eficiências exergéticas do trocador de calor. Essas são dadas pelas equações (3.78) e (3.79)

$$
\begin{aligned}
\varepsilon t c r= & \frac{\dot{n}_{f} \bar{e}_{s f}}{\dot{n}_{f} \bar{e}_{e f}+\dot{n}_{q} \bar{e}_{e q}}, \\
\varepsilon_{t c r}{ }^{+}= & \frac{\dot{n}_{f} \bar{e}_{s f}+\dot{n}_{q} \bar{e}_{s q}}{\dot{n}_{f} \bar{e}_{e f}+\dot{n}_{q} \bar{e}_{e q}} .
\end{aligned}
$$

\subsubsection{Condensador}

Este componente converte o vapor expandido na turbina em líquido para que possa ser novamente comprimido. Como se trata de um trocador de calor, sua modelagem será análoga à utilizada para o trocador de calor no item 3.6.6. 


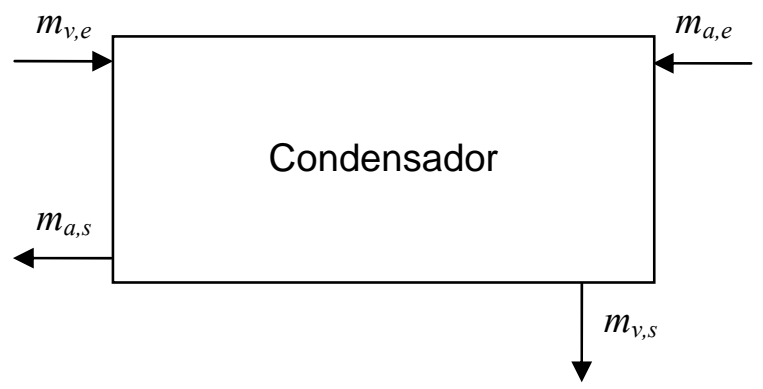

Figura 3.16 Esquema de condensador

\subsubsection{Balanço de energia}

O balanço de energia, considerando-se os fluxos indicados na figura 3.16 e admitindo que o componente é adiabático, é dado pela equação (3.80)

$$
\dot{n}_{v}\left(\bar{h}_{s v}-\bar{h}_{e v}\right)-\dot{n}_{a}\left(\bar{h}_{s a}-\bar{h}_{e a}\right)=0 .
$$

\subsubsection{Efetividade}

A relação auxiliar utilizada neste componente é o conceito de efetividade. A partir dos perfis de temperatura mostrados na figura 3.17 e aplicando-se o conceito de efetividade obtêm-se a equação (3.81). Onde o termo $\bar{h}_{s a, v}$ indica a entalpia do líquido de arrefecimento avaliada na temperatura do vapor.

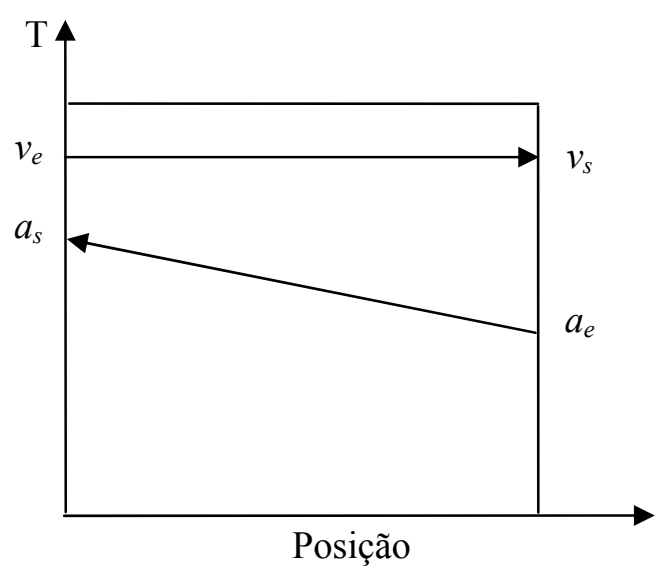

Figura 3.17 - Temperatura no condensador

$$
e f_{c d}=\frac{\bar{h}_{e a}-\bar{h}_{s a}}{\bar{h}_{e a}-\bar{h}_{s a, v}} .
$$

Neste componente não serão consideradas perdas de pressão de forma que a modelagem termodinâmica encerra-se neste ponto.

\subsubsection{Balanço de exergia}

O balanço de exergia é expresso pela equação (3.82)

$$
\dot{n}_{a}\left(\bar{e}_{e a}-\bar{e}_{s a}\right)+\dot{n}_{v}\left(\bar{e}_{e_{v}}-\bar{e}_{s v}\right)-\dot{E}_{D_{c d}}=0
$$


Após o balanço de exergia pode-se classificar os fluxos de exergia presentes no componente como matéria-prima, produtos ou perdas.

Considerando que a função do condensador é aquecer o fluido de arrefecimento, tem-se que:

Matéria-prima: fluxos de massa admitidos no condensador

$$
\dot{E}_{m}=\dot{n}_{v} \bar{e}_{v}+\dot{n}_{a} \bar{e}_{e a}
$$

Produtos: o fluxo de exergia associado à água de arrefecimento que é aquecida e deixa o condensador

$$
\dot{E}_{p}=\dot{n}_{a} \bar{e}_{s a} .
$$

Perdas: o fluxo de exergia associado ao vapor que é condensado e deixa o condensador

$$
\dot{E}_{l}=\dot{n}_{v} \bar{e}_{s v}
$$

Agora pode-se definir as eficiências exergéticas do componente. Essas são dadas pelas equações (3.86) e (3.87)

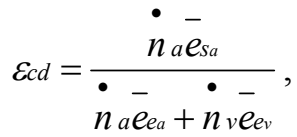

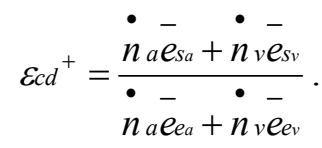

\subsubsection{Bomba de água}

A bomba de água, cuja função é elevar a pressão da água antes que esta seja inserida na caldeira de recuperação é mostrada esquematicamente na figura 3.18 .

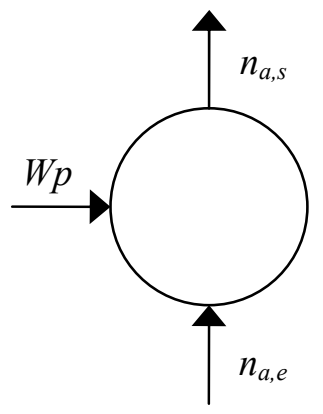

Figura 3.18 - Esquema da bomba de água

\subsubsection{Balanço de energia}


No caso da bomba utiliza-se o conceito de eficiência juntamente com o balanço de energia, de forma que estes dois conceitos apresentem-se em uma única equação. Esta equação (3.88) relaciona a quantidade de energia transferida para o fluido com aquela consumida pela bomba

$$
\eta_{b b}=\frac{\dot{n l}_{l}\left(p_{s l}-p_{e l}\right)}{\dot{\dot{W}}_{p}} .
$$

Esta única equação representa a modelagem termodinâmica da bomba de água, pois o processo neste caso é considerado isotérmico. Além disso, as pressões na entrada e saída são determinadas por fatores externos, de forma que a equação (3.88) é utilizada apenas para determinar a potência consumida pela bomba.

\subsubsection{Balanço de exergia}

A modelagem exergética deste componente é expressa pela equação (3.89)

$$
\dot{n}_{l}\left(\bar{e}_{s l}-\bar{e}_{e l}\right)+\dot{W}_{p}-\dot{E}_{D b b}=0 .
$$

Agora considerando a bomba como um gerador de líquido a alta pressão pode-se definir que fluxos de exergia são matéria-prima, produtos ou perdas. Assim, tem-se que:

Matéria-prima: o trabalho fornecido à bomba e a exergia associada aos fluxos de líquido que entram na bomba

$$
\dot{E}_{m}=\dot{W}_{p}+\dot{n}_{i} \bar{e}_{e l} .
$$

Produtos: a exergia associada aos fluxos de líquido que saem da bomba

$$
\dot{E}_{p}=\dot{n} \bar{l}_{s l} .
$$

Perdas: não ocorrem perdas neste componente.

Agora pode-se definir as eficiências exergéticas da bomba

$$
\varepsilon b b=\varepsilon b b^{+}=\frac{\dot{n_{l} \bar{e}_{s l}}}{\dot{n_{l} \bar{e}_{e l}+\dot{W}_{p}} .}
$$

Neste ponto encerra-se a modelagem dos componentes a serem analisados neste trabalho.

\section{$4 \quad$ Análise da usina termelétrica de Uruguaiana}

A Usina Termelétrica de Uruguaiana é a maior usina térmica a gás natural do país e possui uma capacidade de geração de $600.510 \mathrm{~kW}$. Esta usina é composta por dois blocos de turbina a gás que geram $176.090 \mathrm{~kW}$ de potência cada um e três turbinas a vapor que geram juntas $264.530 \mathrm{~kW}$ de potência. O modelo da turbina presente neste sistema é o W501FC ${ }^{+}$da Siemens Westinghouse. Dentre as principais características deste sistema é válido destacar a utilização de um combustor do tipo DLN ("Dry Low NOx") e um compressor com o dispositivo "Inlet Guide Vanes". O ciclo a 
vapor possui três níveis de pressão no qual o vapor é gerado a $16858 \mathrm{kPa}, 3531 \mathrm{kPa}$ e $501,9 \mathrm{kPa}$. A seguir é apresentado um esquema simplificado da usina de Uruguaiana: 


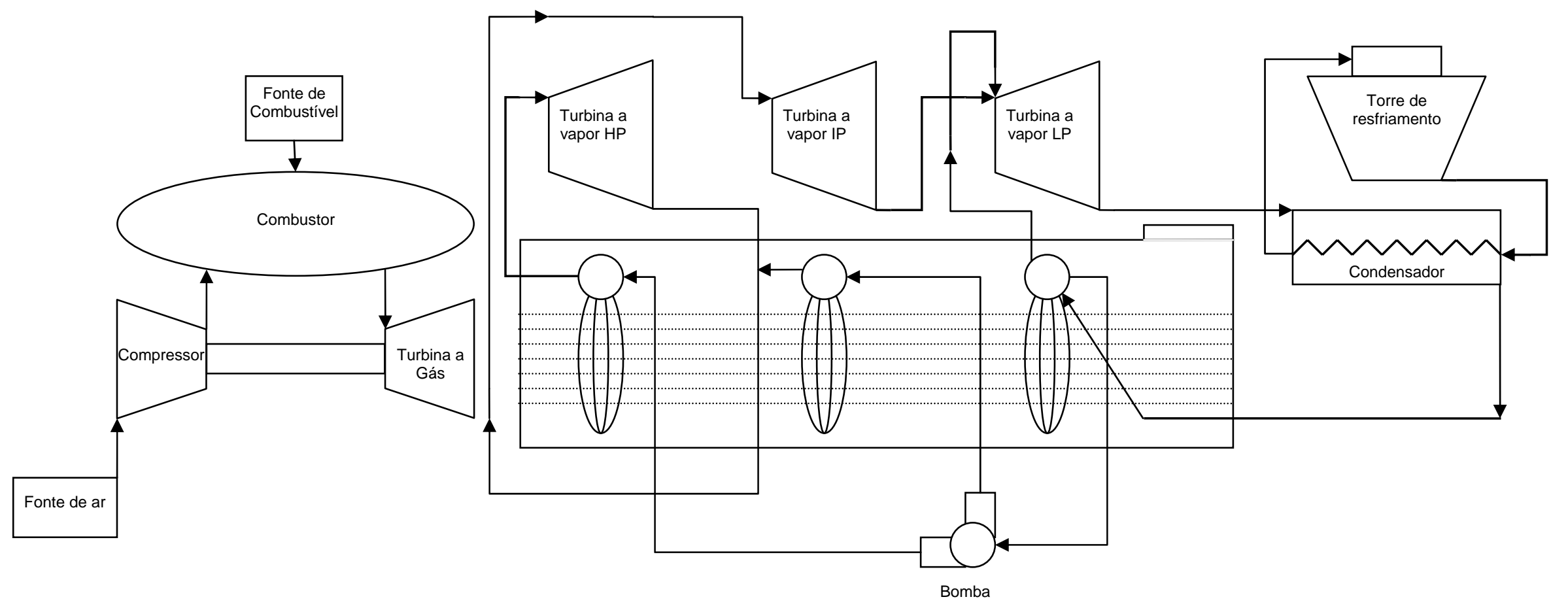

Figura 4.1 - Esquema simplificado da Usina de Uruguaiana 


\subsection{Análise do ciclo}

A maior parte dos dados necessários à análise será informada de acordo com as condições reais de operação do ciclo. Entretanto, algumas considerações deverão ser feitas de acordo com a literatura pesquisada em conformidade com a modelagem de componentes proposta no capítulo 3 .

A presente análise será desenvolvida considerando variações desprezíveis de energia cinética e potencial nos processos envolvidos.

Eventualmente, quando for apresentada a equação para a exergia como propriedade intensiva, poderá ser omitido o termo de exergia química. Fica convencionado que quando esse termo for omitido a exergia química será nula.

A convenção anterior será de grande utilidade pois os processos que envolvem reação química somente ocorrem no combustor e na queima suplementar.

Os cálculos foram desenvolvidos utilizando-se o programa EES Engineering Equation Solver no qual foram utilizadas as bibliotecas para obtenção de propriedades termodinâmicas de substâncias presentes no ciclo analisado. Para melhor orientar o leitor, sempre será informado quando as propriedades forem obtidas através do programa EES através de uma sigla posicionada à direita do texto.

Para identificar fluxos que cruzam o volume de controle serão utilizados os índices $e$ e $s$ para entrada e saída respectivamente.

Por fim, cada vez que for utilizada uma equação esta será identificada através do número definido para esta no capítulo 3. Este número será posicionado à direita da equação mencionada.

A seguir estão listados os principais dados utilizados na análise do ciclo.

4.2 Dados do ciclo de Uruguaiana

- Combustível utilizado: Gás Natural - Metano $\left(\mathrm{CH}_{4}\right)$;

- Poder Calorífico Inferior (LHV) do combustível: 19999 Btu/Lb (46518 kJ/kg);

- Vazão mássica de combustível: $81160 \mathrm{Lb} / \mathrm{hr}(10,23 \mathrm{~kg} / \mathrm{s})$;

- Vazão mássica de ar: $3424540 \mathrm{Lb} / \mathrm{hr}(431,5 \mathrm{~kg} / \mathrm{s})$;

- Vazão mássica de liquido saturado: $1518890 \mathrm{Lb} / \mathrm{hr}(191,4 \mathrm{~kg} / \mathrm{s})$;

- Pressão ambiente: 14,7 psi $(101,3 \mathrm{kPa})$;

- Temperatura ambiente: $60^{\circ} \mathrm{F}\left(15,56^{\circ} \mathrm{C}\right)$;

- Pressão de fornecimento do combustível: 460 psi (3171 kPa);

- Temperatura de fornecimento do combustível (Entrada do Combustor): $265^{\circ} \mathrm{F}$ $\left(129,4^{\circ} \mathrm{C}\right)$

- Temperatura de descarga dos gases na turbina: $1122^{\circ} \mathrm{F}\left(605,6^{\circ} \mathrm{C}\right)$;

- Temperatura de descarga dos gases para a atmosfera: $167,2^{\circ} \mathrm{F}\left(75,11^{\circ} \mathrm{C}\right)$;

- Taxa de transferência de calor por unidade de trabalho: $6474 \mathrm{Btu} / \mathrm{kWhr}$;

- Condição de geração do vapor: $351,6^{\circ} \mathrm{C}$ e $16858 \mathrm{kPa}$ (Alta pressão); $237,6^{\circ} \mathrm{C}$ e 3531 $\mathrm{kPa}$ (Média pressão); $152^{\circ} \mathrm{C}$ e $501,9 \mathrm{kPa}$ (Baixa pressão).

\subsection{Análise do Ciclo Brayton}

Para analisar o ciclo, inicialmente é necessário obter a potência total gerada pela turbina a gás. Para o cálculo da potência será utilizada a equação 4.1,

$$
T_{Q}=\frac{\dot{m_{c b}} \overline{L H V}}{\dot{W}_{c p}+\dot{W}_{l v}} .
$$


Essa equação estabelece a relação entre a quantidade de calor que é fornecida para cada unidade de trabalho disponibilizada pela turbina onde $T_{Q}$ é a taxa de transferência de calor por unidade de trabalho. A relação anterior foi fornecida juntamente com os dados obtidos da usina de Uruguaiana.

Assim, tem-se que

$$
\dot{W}_{c p}+\dot{W}_{l v}=\frac{81160 \cdot 19999}{6474}=250713 \mathrm{~kW}
$$

Como a trabalho líquido já é conhecido, pode-se obter a potência necessária ao compressor

$$
\dot{W}_{c p}=250713-176090=74623 \mathrm{~kW}
$$

O rendimento térmico do ciclo Brayton é definido pela equação 4.2

$$
\eta=\frac{\dot{W}_{l i q}}{Q_{H}}
$$

Onde o rendimento é dado pela relação entre o trabalho líquido e o calor fornecido ao ciclo. Neste caso o calor será assumido como o produto entre a vazão de combustível e o poder calorífico inferior

$$
\eta=\frac{176090}{10,23 \cdot 46518}=0,37
$$

Com essas informações, pode-se iniciar a análise dos componentes do ciclo Brayton.

\subsubsection{Compressor}

O compressor do ciclo de Uruguaiana possui um dispositivo de resfriamento evaporativo antes da entrada que serve para diminuir a temperatura do ar e aumentar a vazão dos gases proporcionando um menor consumo de combustível por $\mathrm{kW}$ gerado e diminuindo a emissão de gases como $\mathrm{NO}_{x}$ e CO. Com a presença do resfriamento, a temperatura a ser utilizada para a entrada no compressor deveria ser a de saturação adiabática.

Como não foi obtida nenhuma informação sobre este dispositivo, será considerado para esta análise que o ar entra no compressor à temperatura de bulbo úmido do ar para a temperatura do ambiente considerado. Ao assumir esse valor não ocorrerão grandes diferenças em relação aos valores reais pois na pressão atmosférica a diferença entre a temperatura de bulbo úmido e a de saturação adiabática é bem pequena. Por fim, será admitido um valor de 0,9 para a umidade relativa do ar. Esse último dado foi obtido junto ao Instituto Nacional de Meteorologia para a região de localização da Usina de Uruguaiana.

\subsubsection{Condições do ambiente}

Fluido de trabalho:

$\mathrm{Ar}$

Entalpia para o ambiente:

$h_{0}=289,1 \mathrm{~kJ} / \mathrm{kg}$

(Tabela de propriedades EES)

Entropia para o ambiente: 
$s_{0}=5,659 \mathrm{~kJ} / \mathrm{kgK}$

Umidade relativa do ar:

$R_{0}=0,9$

\subsubsection{Condições na entrada}

Temperatura na entrada:

$T_{e}=14,55^{\circ} \mathrm{C}$

Pressão na entrada:

$p_{e}=101,3 \mathrm{kPa}$

Entalpia na entrada:

$h_{e}=288 \mathrm{~kJ} / \mathrm{kg}$

Entropia na entrada:

$S_{e}=5,656 \mathrm{~kJ} / \mathrm{kgK}$

4.3.1.3 Parâmetros de análise do componente

Potência consumida:

$\dot{W}_{c p}=74623 \mathrm{~kW}$

Relação de calores específicos:

$k=1,399$ isoentrópica

Como dispõe-se do rendimento do ciclo Brayton, pode-se obter a relação de pressão

$$
\begin{aligned}
0,37 & =1-\frac{1}{R_{\text {cpiso }} \frac{(1,399-1)}{1,399}} \\
R_{\text {cpiso }} & =5,055 \Rightarrow p_{\text {siso }}=5,055 \cdot 101,3=512,2 \mathrm{kPa}
\end{aligned}
$$

Com isso obtêm-se a pressão de saída isoentrópica. Entretanto, afim de obter informações mais condizentes com a realidade, será considerada para a pressão de saída real uma variação dada pelo coeficiente $\emptyset_{c p}$ através da relação

$$
p_{s}=\left(1-\phi_{c p}\right) p_{s i s o}
$$

Para essa análise, será considerado o valor padrão de $\emptyset_{c p}$ igual a 0.05 .

$$
p_{s}=(1-0,05) \cdot 512,2=496,8 \mathrm{kPa}
$$

4.3.1.4 Equações de balanço

Balanço de massa

$$
\begin{aligned}
& \dot{m}_{s}=\dot{m}_{e} \\
& \dot{m}_{s}=\dot{m}_{e}=431,5 \mathrm{~kg} / \mathrm{s}
\end{aligned}
$$


Balanço de energia

$\dot{W}_{c p}+\dot{m}_{e} h_{e}=\dot{m}_{s} h_{s}$

$74623+431,5 \cdot 288,0=431,5 \cdot h_{s}$

$h_{s}=461 \mathrm{~kJ} / \mathrm{kg}$

4.3.1.5 Condições na saída

Temperatura na saída:

$T_{s}=185,7^{\circ} \mathrm{C}$

Entropia na saída:

$s_{s}=5,671 \mathrm{~kJ} / \mathrm{kgK}$

(EES)

4.3.1.6 Análise exergética

Exergia dos fluxos de massa na entrada:

$e_{e}=\left(h_{e}-h_{0}\right)-T_{0}\left(s_{e}-s_{0}\right)$

$e_{e}=(288,0-289,1)-288,7(5,656-5,659)=0,001768 \mathrm{~kJ} / \mathrm{kg}$

Exergia dos fluxos de massa na saída:

$e_{s}=\left(h_{s}-h_{0}\right)-T_{0}\left(s_{s}-s_{0}\right)$

$e_{s}=(461,0-289,1)-288,7(5,671-5,659)=168,6 \mathrm{~kJ} / \mathrm{kg}$

Matéria prima:

$E_{m}=\dot{W}_{c p}+\dot{m}_{e} e_{e}$

$E_{m}=74623+431,5 \cdot 0,001768=74624 \mathrm{~kW}$

Produtos:

$E_{p}=\dot{m} e_{s}$

$E_{p}=431,5 \cdot 168,6=72736 \mathrm{~kW}$

Perdas: Não há perdas neste componente

Exergia dissipada:

$\dot{E}_{d}=\dot{E}_{m}-\left(\dot{E}_{p}+\dot{E}_{l}\right)$

$\dot{E}_{d}=74624-(72736+0)=1888 \mathrm{~kW}$

Eficiência exergética:

$\varepsilon=\frac{\dot{E}_{p}}{\dot{E}_{m}}$

$\varepsilon=\frac{72736}{74624}=0,97$

Eficiência exergética maior: 


$$
\begin{aligned}
\varepsilon^{+} & =\frac{\dot{E}_{p}+\dot{E}_{l}}{\dot{E}_{m}} \\
\varepsilon^{+} & =\frac{72736+0}{74624}=0,97
\end{aligned}
$$

\subsubsection{Combustor}

A análise do combustor envolve uma modelagem do processo de combustão entre o ar e o combustível fornecido. Para este trabalho foi escolhida a equação para a combustão do Metano $\left(\mathrm{CH}_{4}\right)$, pois este elemento apresenta uma fração superior a $95 \%$ em relação à massa total de combustível fornecido. Nesta reação têm-se como reagentes $\mathrm{CH}_{4}, \mathrm{O}_{2}$ e $\mathrm{N}_{2}$. Para os produtos teremos os gases $\mathrm{CO}_{2}, \mathrm{H}_{2} \mathrm{O}, \mathrm{N}_{2}$ e $\mathrm{O}_{2}$. índice $c b$.

Neste componente o ar de reação será identificado pelo índice ar e o combustível pelo

\subsubsection{Condições do ambiente}

Fluido de trabalho:

Ar e Gás Natural (Metano)

Entalpia para o ambiente:

$$
\begin{aligned}
& h_{0_{a r}}=289,1 \mathrm{~kJ} / \mathrm{kg} \\
& h_{0_{c b}}=-4688 \mathrm{~kJ} / \mathrm{kg} \\
& h_{0 \mathrm{co}_{2}}=-8949 \mathrm{~kJ} / \mathrm{kg} \\
& h_{0_{22} \mathrm{O}}=-13440 \mathrm{~kJ} / \mathrm{kg} \\
& h_{0_{\mathrm{N} 2}}=-9,829 \mathrm{~kJ} / \mathrm{kg} \\
& h_{0 \mathrm{O} 2}=-8,713 \mathrm{~kJ} / \mathrm{kg}
\end{aligned}
$$

Entropia para o ambiente:

$$
\begin{aligned}
& s 0_{a r}=5,659 \mathrm{~kJ} / \mathrm{kgK} \\
& s 0_{c b}=11,53 \mathrm{~kJ} / \mathrm{kg} \\
& s 0_{\mathrm{CO}_{2}}=4,826 \mathrm{~kJ} / \mathrm{kgK} \\
& s 0_{\mathrm{H}_{2} O}=10,41 \mathrm{~kJ} / \mathrm{kgK} \\
& s_{0_{N 2}}=6,799 \mathrm{~kJ} / \mathrm{kgK} \\
& s 0_{o_{2}}=6,374 \mathrm{~kJ} / \mathrm{kgK}
\end{aligned}
$$

\subsubsection{Condições na entrada}

Temperatura na entrada:

$$
\begin{aligned}
T_{e a r} & =185,7^{\circ} \mathrm{C} \\
T_{e c b} & =129,4^{\circ} \mathrm{C}
\end{aligned}
$$

Pressão na entrada:

$$
\begin{aligned}
& P_{e a r}=496,8 \mathrm{kPa} \\
& P_{e c b}=3172 \mathrm{kPa}
\end{aligned}
$$

Entalpia na entrada:

$$
\begin{aligned}
& h_{e a r}=461 \mathrm{~kJ} / \mathrm{kg} \\
& h_{e c b}=-4418 \mathrm{~kJ} / \mathrm{kg}
\end{aligned}
$$

Entropia na entrada: 


$$
\begin{aligned}
& S_{e a r}=5,671 \mathrm{~kJ} / \mathrm{kgK} \\
& \operatorname{Sec}_{b}=10,53 \mathrm{~kJ} / \mathrm{kg}
\end{aligned}
$$

4.3.2.3 Parâmetros de análise do componente

Poder Calorífico Inferior:

$$
L H V=46518 \mathrm{~kJ} / \mathrm{kg}
$$

Equação para a combustão do metano:

$$
\mathrm{CH}_{4}+\lambda \cdot 2 \cdot \mathrm{O}_{2}+\lambda \cdot 2 \cdot 3,76 \cdot \mathrm{N}_{2} \rightarrow \mathrm{CO} 2+2 \cdot \mathrm{H}_{2} \mathrm{O}+\lambda \cdot 2 \cdot 3,76 \cdot \mathrm{N}_{2}+(\lambda-1) \cdot 2 \cdot \mathrm{O}_{2}
$$

Nesta equação, $\lambda$ é a relação entre a massa de ar real e a massa de ar teórico necessário à combustão completa. Uma observação importante é que a equação para a combustão acima estabelece a quantidade de produtos e reagentes para $1 \mathrm{~mol}$ de combustível. Conforme estabelecido na modelagem do processo de separação do ar para reação, tem-se a massa de ar fornecida para a reação

$$
\begin{aligned}
& \dot{m}_{a r}=\phi_{a r} \cdot 431,5 \\
& \dot{m}_{a r}=0,6 \cdot 431,5=258,9 \mathrm{~kg} / \mathrm{s}
\end{aligned}
$$

Para se obter a massa de cada produto da reação, necessita-se primeiro obter a quantidade de moles de cada um de acordo com a reação de combustão. A quantidade de moles de combustível e de ar é obtida dividindo a massa de cada um pelo respectivo peso molecular

Moles de Ar:

$$
\dot{m o l}{ }_{a r}=\frac{258,9}{28,97}=8,938 \mathrm{kmol} / \mathrm{s}
$$

Moles de Combustível:

$$
\dot{m o l}_{c b}=\frac{10,23}{16,04}=0,6374 \mathrm{kmol} / \mathrm{s}
$$

Assim, pode-se obter o valor de $\lambda$

$$
\lambda=\frac{258,9}{(1+3,76) \cdot 2 \cdot 0,6374 \cdot 28,97}=1,473
$$

Finalmente pode-se obter a massa de cada produto da combustão multiplicando o número de moles de cada um pelo seu respectivo peso molecular

Massa de $\mathrm{CO}_{2}$ :

$\dot{m C O}_{2}=1 \cdot 0,6374 \cdot 44,01=28,05 \mathrm{~kg} / \mathrm{s}$

$$
\left(\mathrm{M}_{\mathrm{CO} 2}=44,01\right)
$$

Massa de $\mathrm{H}_{2} \mathrm{O}$ :

$$
\dot{m}_{H_{2} \mathrm{O}}=2 \cdot 0,6374 \cdot 18,02=22,97 \mathrm{~kg} / \mathrm{s}
$$$$
\left(\mathrm{M}_{\mathrm{H} 2 \mathrm{O}}=18,02\right)
$$ 
Massa de $\mathrm{N}_{2}$ :

$\dot{m}_{N_{2}}=3,76 \cdot 1,473 \cdot 2 \cdot 0,6374 \cdot 28,01=197,8 \mathrm{~kg} / \mathrm{s}$

$\left(\mathrm{M}_{\mathrm{N} 2}=28,01\right)$

Massa de $\mathrm{O}_{2}$ :

$\dot{m}_{O_{2}}=(1,473-1) \cdot 2 \cdot 0,6374 \cdot 32=19,29 \mathrm{~kg} / \mathrm{s}$

$\left(\mathrm{M}_{\mathrm{O} 2}=32\right)$

4.3.2.4 Equações de balanço

Balanço de massa

$\dot{m}_{a r}+\dot{m}_{c b}=\dot{m}_{\mathrm{CO}_{2}}+\dot{m}_{\mathrm{H}_{2} \mathrm{O}}+\dot{m}_{\mathrm{N}_{2}}+\dot{m}_{\mathrm{O}_{2}}$

Esses dados já foram calculados no item anterior.

Balanço de energia

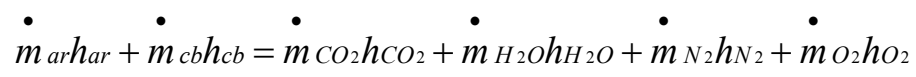

4.3.2.5 Condições na saída do combustor

Pressão na saída do combustor:

$$
\begin{aligned}
& p_{g r}=\left(1-\phi_{p c c}\right) p_{a d} \\
& p_{g r}=(1-0,05) \cdot 496,8=472 \mathrm{kPa}
\end{aligned}
$$

Neste caso, o balanço de energia será uma equação necessária para se encontrar a temperatura de saída dos produtos da reação. Admitindo que todos os produtos estarão à mesma temperatura na saída do combustor, obtêm-se as entalpias de cada um à uma temperatura fixada inicialmente. Em seguida verifica-se se foi mantida a condição de equilíbrio para a equação da energia. Caso contrário atribui-se um novo valor para a temperatura de saída até que o equilíbrio de energia seja verificado.

Como temperatura inicial, sugere-se utilizar a temperatura adiabática de chama para a combustão do Combustível, pois essa seria uma temperatura que ocorreria em um processo ideal onde toda a energia dos reagentes estaria presente nos produtos. Utilizando-se a temperatura adiabática de chama, o processo iterativo seria estabelecido através de incrementos que diminuíssem a temperatura a cada verificação feita na equação da energia. Como dispõe-se de um programa com todas as propriedades termodinâmicas das substâncias já armazenadas em bibliotecas internas, pode-se recorrer à suas funções internas e obter a temperatura de saída admitindo que esta seja uma variável cujo valor será encontrado quando a condição do balanço de energia for estabelecida.

Para os dados fornecidos ao programa (EES) foram obtidos os seguintes valores para as propriedades na saída do combustor:

Entalpia do $\mathrm{CO}_{2}$ :

$h \mathrm{CO}_{2}=-6683 \mathrm{~kJ} / \mathrm{kg}$

Entalpia do $\mathrm{H}_{2} \mathrm{O}$ :

$h_{H_{2} O}=-9011 \mathrm{~kJ} / \mathrm{kg}$

Entalpia do $\mathrm{N}_{2}$ :

$h_{N_{2}}=2174 \mathrm{~kJ} / \mathrm{kg}$

Entalpia do $\mathrm{O}_{2}$ : 
$h_{o_{2}}=2007 \mathrm{~kJ} / \mathrm{kg}$

Entropia do $\mathrm{CO}_{2}$ :

$\mathrm{SCO}_{2}=6,819 \mathrm{~kJ} / \mathrm{kgK}$

Entropia do $\mathrm{H}_{2} \mathrm{O}$ :

$\mathrm{SH}_{2} \mathrm{O}=14,15 \mathrm{~kJ} / \mathrm{kgK}$

Entropia do $\mathrm{N}_{2}$ :

$S N_{2}=8,617 \mathrm{~kJ} / \mathrm{kgK}$

Entropia do $\mathrm{O}_{2}$ :

$S_{2}=8,068 \mathrm{~kJ} / \mathrm{kgK}$

Temperatura dos produtos na saída do combustor:

$T_{g r}=1859^{\circ} \mathrm{C}$

Pode-se observar que a temperatura encontrada é bastante alta e certamente não poderia ser suportada pelos equipamentos utilizados no ciclo. Entretanto, estabeleceu-se a hipótese de que esses produtos ainda serão resfriados através da mistura entre esses e o ar destinado ao processo de mistura, antes de entrarem na turbina.

\subsubsection{Análise exergética}

Exergia dos fluxos de massa na entrada:

$$
\begin{aligned}
& e_{a r}=\left(h_{a r}-h_{a r}\right)-T_{0}\left(s a r-s 0_{a r}\right) \\
& e_{c b}=\left(h_{c b}-h_{c b}\right)-T_{0}\left(s c b-s 0_{c b}\right)+L H V \\
& e_{a r}=(461-289,1)-288,7(5,671-5,659)=168,6 \mathrm{~kJ} / \mathrm{kg} \\
& e_{c b}=((-4418)-(-4688))-288,7(10,53-11,53)+46518=47076 \mathrm{~kJ} / \mathrm{kg}
\end{aligned}
$$

Exergia dos fluxos de massa na saída:

$$
\begin{aligned}
& e \mathrm{CO}_{2}=\left(h_{\mathrm{CO}_{2}}-h_{0 \mathrm{CO} 2}\right)-T_{0}\left(\mathrm{SCO}_{2}-\mathrm{SOCO}_{2}\right) \\
& e_{\mathrm{H}_{2} \mathrm{O}}=\left(h_{\mathrm{H}_{2} \mathrm{O}}-h_{\mathrm{H}_{2} \mathrm{O}}\right)-\mathrm{T}_{0}\left(\mathrm{SH}_{2} \mathrm{O}-\mathrm{SOH}_{2} \mathrm{O}\right) \\
& e_{N_{2}}=\left(h_{N_{2}}-h_{0 N_{2}}\right)-T_{0}\left(s_{N_{2}}-S_{N_{2}}\right) \\
& e \mathrm{O}_{2}=\left(\mathrm{hO}_{2}-\mathrm{h}_{\mathrm{OO}}\right)-\mathrm{T}_{0}\left(\mathrm{SO}_{2}-\mathrm{SOO}_{2}\right) \\
& e \mathrm{CO}_{2}=((-6683)-(-8949))-288,7(6,819-4,826)=1692 \mathrm{~kJ} / \mathrm{kg} \\
& e_{\mathrm{H}_{2} \mathrm{O}}=((-9011)-(-13440))-288,7(14,15-10,41)=3348 \mathrm{~kJ} / \mathrm{kg} \\
& e_{N_{2}}=(2174-(-9,829))-288,7(8,617-6,799)=1659 \mathrm{~kJ} / \mathrm{kg} \\
& e_{2}(2007-(-8,713))-288,7(8,068-6,374)=1527 \mathrm{~kJ} / \mathrm{kg}
\end{aligned}
$$

Matéria prima:

$$
\begin{aligned}
& E_{m}=\dot{m}_{\text {are }} \quad \dot{m}_{c b} e_{c b} \\
& E_{m}=258,9 \cdot 168,6+10,23 \cdot 47076=525046 \mathrm{~kW}
\end{aligned}
$$

Produtos:

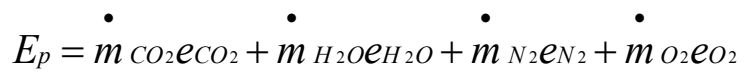

$E_{p}=28,05 \cdot 1692+22,97 \cdot 3348+197,8 \cdot 1659+19,29 \cdot 1527=481822 \mathrm{~kW}$

Perdas: Não há perdas neste componente 
Exergia dissipada:

$$
\begin{aligned}
& \dot{E}_{d}=\dot{E}_{m}-\left(\dot{E}_{p}+\dot{E}_{l}\right) \\
& \dot{E}_{d}=525046-(481822+0)=43224 \mathrm{~kW}
\end{aligned}
$$

Eficiência exergética:

$$
\begin{aligned}
& \varepsilon=\frac{\dot{E}_{p}}{\dot{E}_{m}} \\
& \varepsilon=\frac{481822}{525046}=0,92
\end{aligned}
$$

Eficiência exergética maior:

$$
\begin{aligned}
\varepsilon^{+} & =\frac{\dot{E}_{p}+\dot{E}_{l}}{\dot{E}_{m}} \\
\varepsilon^{+} & =\frac{481822+0}{525046}=0,92
\end{aligned}
$$

\subsubsection{Turbina a Gás}

\subsubsection{Condições do ambiente}

Fluido de trabalho:

Ar e Produtos da reação no combustor $\left(\mathrm{CO}_{2}, \mathrm{H}_{2} \mathrm{O}, \mathrm{N}_{2}, \mathrm{O}_{2}\right)$

Entalpia para o ambiente:

$$
\begin{aligned}
& h_{0_{a m}}=289,1 \mathrm{~kJ} / \mathrm{kg} \\
& h_{0 \mathrm{CO}_{2}}=-8949 \mathrm{~kJ} / \mathrm{kg} \\
& h_{0_{22}}=-13440 \mathrm{~kJ} / \mathrm{kg} \\
& h_{\mathrm{N}_{2}}=-9,829 \mathrm{~kJ} / \mathrm{kg} \\
& h_{0 \mathrm{o}_{2}}=-8,713 \mathrm{~kJ} / \mathrm{kg}
\end{aligned}
$$

Entropia para o ambiente:

$$
\begin{aligned}
& s 0_{\text {am }}=5,659 \mathrm{~kJ} / \mathrm{kgK} \\
& s 0_{\text {со } 2}=4,826 \mathrm{~kJ} / \mathrm{kgK} \\
& s 0_{\text {H } 2}=10,41 \mathrm{~kJ} / \mathrm{kgK} \\
& s 0_{N 2}=6,799 \mathrm{~kJ} / \mathrm{kgK} \\
& s 0_{O_{2}}=6,374 \mathrm{~kJ} / \mathrm{kgK}
\end{aligned}
$$

\subsubsection{Processo de mistura}

Antes de definir a temperatura de entrada na turbina, é necessário calcular o balanço de massa para o processo de mistura antes dos gases entrarem na turbina.

Balanço de massa para o processo de mistura

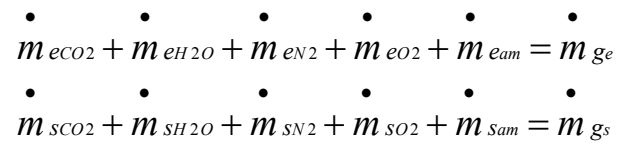


$\dot{m}_{g_{e}}=\dot{m}_{g_{s}}=28,05+22,97+197,8+19,29+172,6=440,7 \mathrm{~kg} / \mathrm{s}$

Balanço de energia para o processo de mistura

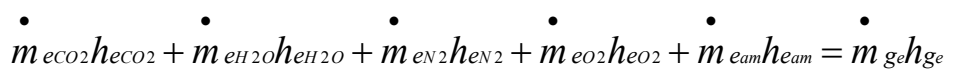

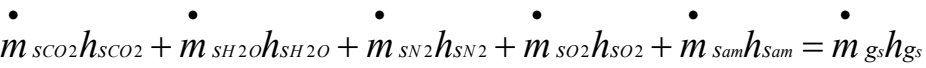

$$
\begin{aligned}
& \dot{m}_{g_{e}} h_{g_{e}}=\dot{m} g_{s} h_{g_{s}} \\
& \dot{m}_{g_{e}} h_{g_{e}}=28,05 \cdot(-6683)+22,97 \cdot(-9013)+197,8 \cdot 2173+19,29 \cdot 2007+172,6 \cdot 461=153616,27 \mathrm{~kW} \\
& m_{g_{s}} h_{g_{s}}=153616,27 \mathrm{~kW}
\end{aligned}
$$

\subsubsection{Condições na entrada da turbina}

Da mesma forma feita para o combustor, será admitido que todas as substâncias após o processo de mistura estarão á mesma temperatura e o balanço de energia deverá ser verificado. Assim, foram obtidos os seguintes valores pelo programa EES

Temperatura dos gases na entrada da turbina:

$$
T_{g_{e}}=1286^{\circ} \mathrm{C}
$$

Pressão na entrada da turbina:

$$
\begin{aligned}
& p_{g_{e}}=\operatorname{MÍN}\left(p_{a m} ; p_{g r}\right) \\
& p_{g_{e}}=\operatorname{MÍN}(496,8 ; 472)=472 \mathrm{kPa}
\end{aligned}
$$

Entalpia na entrada da turbina:

$$
\begin{aligned}
& h_{\text {eam }}=1707 \mathrm{~kJ} / \mathrm{kg} \\
& h_{\text {есо } 2}=-7462 \mathrm{~kJ} / \mathrm{kg} \\
& h_{\text {ен } 2 \mathrm{O}}=-10596 \mathrm{~kJ} / \mathrm{kg} \\
& h_{\text {eN } 2}=1445 \mathrm{~kJ} / \mathrm{kg} \\
& h_{\text {eо } 2}=1336 \mathrm{~kJ} / \mathrm{kg}
\end{aligned}
$$

Entropia na entrada da turbina:

$$
\begin{aligned}
& S_{\text {eam }}=7,046 \mathrm{~kJ} / \mathrm{kgK} \\
& \mathrm{Seco}_{2}=6,393 \mathrm{~kJ} / \mathrm{kgK} \\
& \text { Sен } 2 \text { о }=13,29 \mathrm{~kJ} / \mathrm{kgK} \\
& S_{\text {eN } 2}=8,219 \mathrm{~kJ} / \mathrm{kgK} \\
& \text { Seo } 2=7,702 \mathrm{~kJ} / \mathrm{kgK}
\end{aligned}
$$

4.3.3.4 Parâmetros de análise do componente

Potência do compressor:

$\dot{W}_{c p}=74623 \mathrm{~kW}$

Potência útil:

$$
\dot{W}_{l v}=176090 \mathrm{~kW}
$$

4.3.3.5 Condições na saída da turbina 
Temperatura na saída da turbina:

$T_{g s}=605,6^{\circ} \mathrm{C}$

Pressão na saída da turbina:

$p_{s}=168,3 \mathrm{kPa}$ (Este valor foi calculado de acordo com as condições dadas para a entrada e saída dos gases para a atmosfera)

Entalpia na saída da turbina:

$$
\begin{aligned}
& h_{\text {sam }}=909,2 \mathrm{~kJ} / \mathrm{kg} \\
& h_{\text {sCO2 }}=-8331 \mathrm{~kJ} / \mathrm{kg} \\
& h_{\text {SH } 20}=-12252 \mathrm{~kJ} / \mathrm{kg} \\
& h_{\text {SN } 2}=627,1 \mathrm{~kJ} / \mathrm{kg} \\
& h_{\text {SO } 2}=578,7 \mathrm{~kJ} / \mathrm{kg}
\end{aligned}
$$

Entropia na saída da turbina:

$$
\begin{aligned}
& S_{\text {sam }}=6,672 \mathrm{~kJ} / \mathrm{kgK} \\
& S_{s c 02}=5,86 \mathrm{~kJ} / \mathrm{kgK} \\
& S_{S H 2 O}=12,38 \mathrm{~kJ} / \mathrm{kgK} \\
& s_{s 2}=7,839 \mathrm{~kJ} / \mathrm{kgK} \\
& s_{s o 2}=7,334 \mathrm{~kJ} / \mathrm{kgK}
\end{aligned}
$$

4.3.3.6 Equações de balanço

Balanço de massa

$$
\begin{aligned}
& \dot{m}_{g_{s}}=\dot{m}_{g_{e}} \\
& \dot{m}_{g_{s}}=440,7 \mathrm{~kg} / \mathrm{s}
\end{aligned}
$$

Balanço de energia

$$
\dot{m}_{g_{e}} h_{g_{e}}=\sum \dot{m}_{g_{s i}} h_{g s i}+\dot{W}_{c p}+\dot{W}_{l v}\left(i=a m, \mathrm{CO}_{2}, \mathrm{H}_{2} \mathrm{O}, \mathrm{N}_{2}, \mathrm{O}_{2}\right)
$$

\subsubsection{Análise exergética}

Exergia dos fluxos de massa na entrada:

$$
\begin{aligned}
& e_{e i}=\left(h_{e_{i}}-h_{0_{i}}\right)-T_{0}\left(S_{e_{i}}-S_{0 i}\right),\left(i=a m, \mathrm{CO}_{2}, \mathrm{H}_{2} \mathrm{O}, \mathrm{N}_{2}, \mathrm{O}_{2}\right) \\
& e_{e a m}=(1707-289,1)-288,7(7,045-5,659)=1018 \mathrm{~kJ} / \mathrm{kg} \\
& e_{\text {eсо } 2}=((-7462)-(-8949))-288,7(6,393-4,826)=1035 \mathrm{~kJ} / \mathrm{kg} \\
& e_{\text {ен } 2 \mathrm{O}}=((-10596)-(-13440))-288,7(13,29-10,41)=2012 \mathrm{~kJ} / \mathrm{kg} \\
& e_{\text {eN } 2}=(1445-(-9,829))-288,7(8,219-6,799)=1045 \mathrm{~kJ} / \mathrm{kg} \\
& e_{\text {ео } 2}=(1336-(-8,713))-288,7(7,702-6,374)=961,3 \mathrm{~kJ} / \mathrm{kg}
\end{aligned}
$$

Exergia dos fluxos de massa na saída:

$$
\begin{aligned}
& e_{s i}=\left(h_{s i}-h_{0 i}\right)-T_{0}\left(S_{s i}-S_{0_{i}}\right),\left(i=a m, \mathrm{CO}_{2}, \mathrm{H}_{2} \mathrm{O}, \mathrm{N}_{2}, \mathrm{O}_{2}\right) \\
& e_{s a m}=(909,2-289,1)-288,7(6,672-5,659)=327,8 \mathrm{~kJ} / \mathrm{kg} \\
& e_{s \mathrm{sO}_{2}}=((-8331)-(-8949))-288,7(5,86-4,826)=320,1 \mathrm{~kJ} / \mathrm{kg} \\
& e_{s \mathrm{~S} 2 O}=((-12252)-(-13440))-288,7(12,38-10,41)=619,1 \mathrm{~kJ} / \mathrm{kg}
\end{aligned}
$$




$$
\begin{aligned}
& e_{S N_{2}}=(627,1-(-9,829))-288,7(7,839-6,799)=336,6 \mathrm{~kJ} / \mathrm{kg} \\
& e_{s o_{2}}=(578,7-(-8,713))-288,7(7,334-6,374)=310,3 \mathrm{~kJ} / \mathrm{kg}
\end{aligned}
$$

Matéria prima:

$$
\begin{aligned}
& E_{m}=\sum \dot{m}_{i} e_{e i}\left(i=a m, \mathrm{CO}_{2}, \mathrm{H}_{2} \mathrm{O}, \mathrm{N}_{2}, \mathrm{O}_{2}\right) \\
& E_{m}=172,6 \cdot 1018+28,05 \cdot 1035+22,97 \cdot 2012+197,8 \cdot 1045+19,29 \cdot 961,3=476162 \mathrm{~kW}
\end{aligned}
$$

Produtos:

$$
\begin{aligned}
& E_{p}=\dot{W}_{c p}+\dot{W}_{l v} \\
& E_{p}=74623+176090=250713 \mathrm{~kW}
\end{aligned}
$$

Perdas:

$$
\begin{aligned}
& E_{l}=\sum \dot{m}_{i} e_{s i},\left(i=a m, \mathrm{CO}_{2}, \mathrm{H}_{2} \mathrm{O}, \mathrm{N}_{2}, \mathrm{O}_{2}\right) \\
& E_{l}=172,6 \cdot 327,8+28,05 \cdot 320,1+22,97 \cdot 619,1+197,8 \cdot 336,6+19,29 \cdot 310,3=152352 \mathrm{~kW}
\end{aligned}
$$

Exergia dissipada:

$$
\begin{aligned}
& \dot{E}_{d}=\dot{E}_{m}-\left(\dot{E}_{p}+\dot{E}_{l}\right) \\
& \dot{E}_{d}=476162-(250713+152352)=73097 \mathrm{~kW}
\end{aligned}
$$

Eficiência exergética:

$$
\begin{aligned}
& \varepsilon=\frac{\dot{E}_{p}}{\dot{E}_{m}} \\
& \varepsilon=\frac{250713}{476162}=0,53
\end{aligned}
$$

Eficiência exergética maior:

$$
\begin{aligned}
& \varepsilon^{+}=\frac{\dot{E}_{p}+\dot{E}_{l}}{\dot{E}_{m}} \\
& \varepsilon^{+}=\frac{250713+152352}{476162}=0,85
\end{aligned}
$$

\subsection{Análise do Ciclo Rankine}

No ciclo de Uruguaiana, os gases que saem da turbina atravessam um compartimento que contém vários trocadores de calor tais como economizador, gerador de vapor e superaquecedor.

A fim de elaborar uma análise mais detalhada do ciclo, optou-se por analisar volumes de controle que envolvessem esses trocadores de calor a cada tomada de temperatura dos gases aquecidos. Dessa forma pode-se fechar o balanço tanto de massa como de energia sem ter de recorrer a modelagens uma vez que todas as informações reais estarão disponíveis.

No entanto, em relação à caldeira será considerado somente o gerador de vapor como o volume de controle e assim não será aplicada integralmente a modelagem proposta no capítulo 3 para este componente especificamente.

\subsubsection{Trocadores de calor}


Os geradores de vapor da usina de Uruguaiana operam em três níveis de pressão e fornecem o fluido de trabalho para três turbinas a vapor. Para distinguir cada fluxo de massa de vapor que entra ou sai do volume de controle, foram atribuídos os índices $l p, m p$ e $h p$ para identificar os fluxos em baixa pressão, média pressão e alta pressão respectivamente. Também é extraída uma certa quantidade de líquido saturado do gerador de vapor de baixa pressão para aquecer o combustível antes de entrar no combustor, esse fluxo está identificado pelo índice $c b$. Por último será considerado um fluxo de vapor que é extraído da turbina de alta pressão para se unir ao vapor gerado em média pressão.

Também há uma queima suplementar para aumentar a energia fornecida à caldeira, incorrendo em um aumento na vazão mássica dos gases a partir do ponto 4 do compartimento elevando a vazão mássica dos gases em $0,4 \%$ e um aumento da temperatura neste ponto para $1248,1^{\circ} \mathrm{F}\left(675,6^{\circ} \mathrm{C}\right)$.

A variação da pressão dos gases em decorrência do fluxo através dos dutos e equipamentos será definida por um coeficiente de perda de carga denominado por $\phi_{p c}$. Para este coeficiente será adotado o valor de 0,03 a cada passagem através de um gerador de vapor, economizador ou superaquecedor. Caso seja necessário definir quedas de pressão no lado do vapor será adotado o valor de 0,05 . Por último será considerada uma parcela de perda de calor $\phi_{q}$ igual 0,02 atribuída aos gases caso a troca de calor ocorra a uma temperatura acima de $573 \mathrm{~K}$.

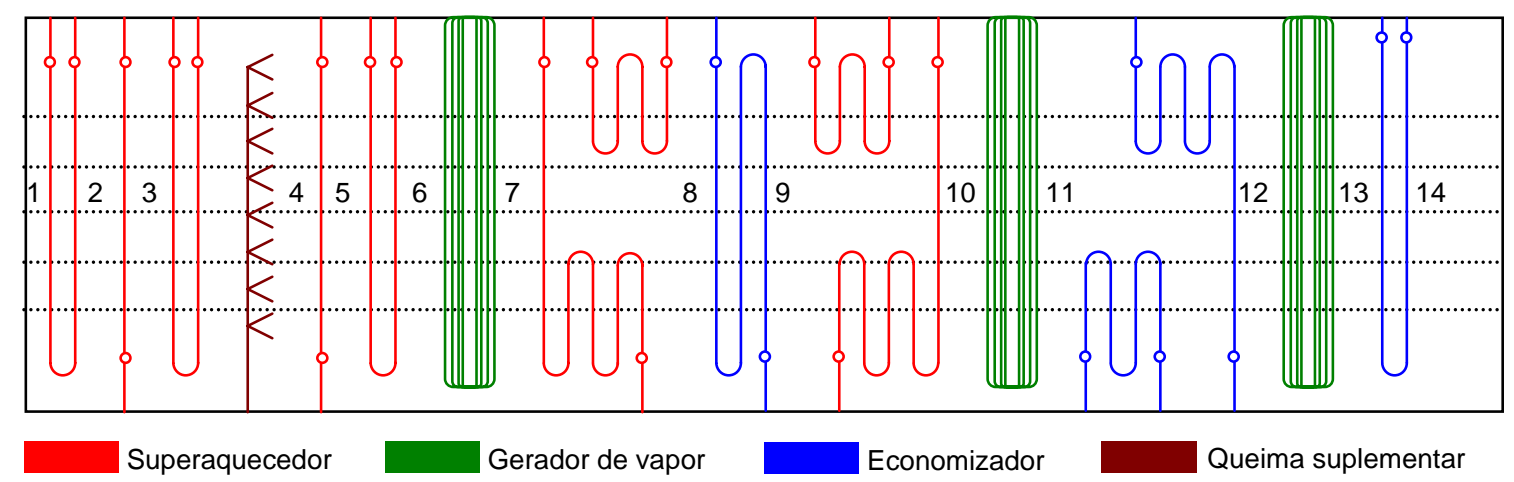

Figura 4.2 - Esquema do corredor de passagem dos gases aquecidos

Através da figura 4.2 acima pode-se identificar os pontos onde foram tomadas as temperaturas dos gases e a qual tipo de trocador de calor a análise se refere.

\subsubsection{Condições do ambiente}

Fluido de trabalho:

$\mathrm{Ar}$, gases da saída da turbina e vapor $\left(\mathrm{am}, \mathrm{CO}_{2}, \mathrm{H}_{2} \mathrm{O}, \mathrm{N}_{2}, \mathrm{O}_{2}\right.$, v)

Entalpia para o ambiente:

$$
\begin{aligned}
& h_{0_{a m}}=289,1 \mathrm{~kJ} / \mathrm{kg} \\
& h_{0_{\mathrm{CO} 2}}=-8949 \mathrm{~kJ} / \mathrm{kg} \\
& h_{0_{\mathrm{H} 2}}=-13440 \mathrm{~kJ} / \mathrm{kg} \\
& h_{0_{\mathrm{N} 2}}=-9,829 \mathrm{~kJ} / \mathrm{kg} \\
& h_{0_{2}}=-8,713 \mathrm{~kJ} / \mathrm{kg} \\
& h_{0_{v}}=65,34 \mathrm{~kJ} / \mathrm{kg}
\end{aligned}
$$

Entropia para o ambiente:

$$
\begin{aligned}
& S_{0 a m}=5,659 \mathrm{~kJ} / \mathrm{kgK} \\
& S_{0 \mathrm{CO} 2}=4,826 \mathrm{~kJ} / \mathrm{kgK} \\
& S_{0 \mathrm{H}_{2 O}}=10,41 \mathrm{~kJ} / \mathrm{kgK}
\end{aligned}
$$




$$
\begin{aligned}
& s 0_{N_{2}}=6,799 \mathrm{~kJ} / \mathrm{kgK} \\
& s 0 o_{2}=6,374 \mathrm{~kJ} / \mathrm{kgK} \\
& s 0_{v}=0,2323 \mathrm{~kJ} / \mathrm{kgK}
\end{aligned}
$$

4.4.1.2 Volume de controle entre os pontos 1 e 2 (Superaquecedor $\mathrm{HP}-3^{\circ}$ passo)

\subsection{Condições na entrada}

Temperatura na entrada:

$$
T_{e_{g}}=605,6^{\circ} \mathrm{C}
$$

$T_{e n p}=499,3^{\circ} \mathrm{C}$

Pressão na entrada:

$$
p_{\text {ehp }}=16499 \mathrm{kPa}
$$
equação

Para calcular a pressão dos gases na entrada do volume de controle será utilizada a seguinte

$$
p_{g_{i}}=\frac{103,3}{\left(1-\phi_{e c}\right)^{n_{e c}} \cdot\left(1-\phi_{g v}\right)^{n_{g v}} \cdot\left(1-\phi_{s a}\right)^{n_{s a}}}
$$

Neste caso, foi fixada a pressão de $103,3 \mathrm{kPa}$ para a saída dos gases para a atmosfera, $\emptyset_{e c}$, $\emptyset_{g v}$, e $\emptyset_{s a}$ são os coeficientes de perda de pressão para o economizador, gerador de vapor e superaquecedor respectivamente. A cada ponto $i=1,2, \ldots, 14$ pode-se calcular a respectiva pressão bastando somente fornecer o número de economizadores $n_{e c}$, geradores de vapor $n_{g v} \mathrm{e}$ superaquecedores $n_{s a}$ entre o ponto considerado e a saída dos gases para a atmosfera.

Para o volume de controle em questão tem-se que

$$
p_{e_{g}}=\frac{103,3}{(1-0,03)^{4} \cdot(1-0,03)^{3} \cdot(1-0,03)^{9}}=168,3 \mathrm{kPa}
$$

Entalpia na entrada:

Considerando que esta troca de calor ocorra a uma temperatura superior a $573 \mathrm{~K}$, a entalpia sofrerá uma redução em seu valor, dada pelo coeficiente de perda de calor. Assim, tem-se a seguir os valores das entalpias obtidos pelo programa EES já reduzidos pelo coeficiente igual a 0,02.

$$
\begin{aligned}
& h_{\text {eam }}=891 \mathrm{~kJ} / \mathrm{kg} \\
& h_{\text {eсо } 2}=-8164 \mathrm{~kJ} / \mathrm{kg} \\
& h_{\text {ен } 2 О}=-12007 \mathrm{~kJ} / \mathrm{kg} \\
& h_{\text {eN } 2}=614,5 \mathrm{~kJ} / \mathrm{kg} \\
& h_{\text {eо } 2}=567,1 \mathrm{~kJ} / \mathrm{kg} \\
& h_{\text {ehp }}=3287 \mathrm{~kJ} / \mathrm{kg}
\end{aligned}
$$

Entropia na entrada:

$$
\begin{aligned}
& S_{\text {eam }}=6,672 \mathrm{~kJ} / \mathrm{kgK} \\
& S_{\text {eсо } 2}=5,86 \mathrm{~kJ} / \mathrm{kgK} \\
& S_{\text {eн } 20}=12,38 \mathrm{~kJ} / \mathrm{kgK} \\
& S_{\text {eN } 2}=7,839 \mathrm{~kJ} / \mathrm{kgK} \\
& S_{e о 2}=7,334 \mathrm{~kJ} / \mathrm{kgK} \\
& S_{\text {enp }}=6,278 \mathrm{~kJ} / \mathrm{kgK}
\end{aligned}
$$




\subsection{Equações de balanço}

Balanço de Massa

Valores de vazão mássica associada a cada substância presente no volume de controle considerado.

$$
\begin{aligned}
& \dot{m}_{\text {eam }}=172,6 \mathrm{~kg} / \mathrm{s} \\
& \dot{m}_{e \mathrm{CO} 2}=28,05 \mathrm{~kg} / \mathrm{s} \\
& \dot{m}_{\text {eH } 2 O}=22,97 \mathrm{~kg} / \mathrm{s} \\
& \dot{m}_{e N 2}=197,8 \mathrm{~kg} / \mathrm{s} \\
& \dot{m}_{e O 2}=19,29 \mathrm{~kg} / \mathrm{s} \\
& \dot{m}_{e h p}=88,24 \mathrm{~kg} / \mathrm{s} \\
& \dot{m}_{s i}=\dot{m}_{e i},\left(\mathrm{i}=\mathrm{hp}, \mathrm{am} ; \mathrm{CO}_{2} ; \mathrm{H}_{2} \mathrm{O} ; \mathrm{N}_{2} ; \mathrm{O}_{2}\right)
\end{aligned}
$$

Balanço de energia

Da mesma forma como no balanço de massa, não será necessário determinar nenhuma variável pelo balanço de energia para este volume de controle.

\subsection{Condições na saída}

Temperatura na saída:

$T_{s_{g}}=590,1^{\circ} \mathrm{C}$

$T_{s h p}=529,3{ }^{\circ} \mathrm{C}$

Pressão na saída:

$$
\begin{aligned}
& p_{s h p}=16320 \mathrm{kPa} \\
& p_{s_{g}}=\frac{103,3}{(1-0,03)^{4} \cdot(1-0,03)^{3} \cdot(1-0,03)^{8}}=163,2 \mathrm{kPa}
\end{aligned}
$$

Entalpia na saída:

$$
\begin{aligned}
& h_{\text {sam }}=874,2 \mathrm{~kJ} / \mathrm{kg} \\
& h_{\text {sCO2 }}=-8182 \mathrm{~kJ} / \mathrm{kg} \\
& h_{\text {sH } 2 O}=-12040 \mathrm{~kJ} / \mathrm{kg} \\
& h_{S \mathrm{~N}^{2}}=597,2 \mathrm{~kJ} / \mathrm{kg} \\
& h_{\text {sO2 } 2}=551 \mathrm{~kJ} / \mathrm{kg} \\
& h_{\text {shp }}=3377 \mathrm{~kJ} / \mathrm{kg}
\end{aligned}
$$

Entropia na saída:

$$
\begin{aligned}
& S_{\text {Sam }}=6,661 \mathrm{~kJ} / \mathrm{kgK} \\
& S_{s \mathrm{CO}_{2}}=5,844 \mathrm{~kJ} / \mathrm{kgK} \\
& S_{S \mathrm{H} 2 \mathrm{O}}=12,36 \mathrm{~kJ} / \mathrm{kgK} \\
& S_{S \mathrm{~N}^{2}}=7,828 \mathrm{~kJ} / \mathrm{kgK} \\
& S_{\text {so } 2}=7,323 \mathrm{~kJ} / \mathrm{kgK} \\
& S_{S h p}=6,397 \mathrm{~kJ} / \mathrm{kgK}
\end{aligned}
$$

\subsection{Análise exergética}


Exergia dos fluxos de massa na entrada:

$$
\begin{aligned}
& e_{e_{i}}=\left(h_{e_{i}}-h_{0 i}\right)-T_{0}\left(s_{e_{i}}-s 0_{i}\right),\left(\mathrm{i}=\mathrm{hp}, \mathrm{am}, \mathrm{CO}_{2}, \mathrm{H}_{2} \mathrm{O}, \mathrm{N}_{2}, \mathrm{O}_{2}\right) \\
& e_{\text {eam }}=(891-289,1)-288,7(6,672-5,659)=309,6 \mathrm{~kJ} / \mathrm{kg} \\
& e_{e \text { со } 2}=((-8164)-(-8949))-288,7(5,86-4,826)=486,7 \mathrm{~kJ} / \mathrm{kg} \\
& e_{\text {eн } 20}=((-12007)-(-13440))-288,7(12,38-10,41)=864,1 \mathrm{~kJ} / \mathrm{kg} \\
& e_{e N 2}=(614,5-(-9,829))-288,7(7,839-6,799)=324,1 \mathrm{~kJ} / \mathrm{kg} \\
& e_{e о 2}=(567,1-(-8,713))-288,7(7,334-6,374)=298,8 \mathrm{~kJ} / \mathrm{kg} \\
& e_{\text {enp }}=(3287-65,34)-288,7(6,278-0,2323)=1476 \mathrm{~kJ} / \mathrm{kg}
\end{aligned}
$$

Exergia dos fluxos de massa na saída:

$$
\begin{aligned}
& e_{s i}=\left(h_{s i}-h_{0 i}\right)-T_{0}\left(s_{s i}-s 0_{i}\right),\left(\mathrm{i}=\mathrm{hp}, \mathrm{am}, \mathrm{CO}_{2}, \mathrm{H}_{2} \mathrm{O}, \mathrm{N}_{2}, \mathrm{O}_{2}\right) \\
& e_{\text {sam }}=(874,2-289,1)-288,7(6,661-5,659)=295,9 \mathrm{~kJ} / \mathrm{kg} \\
& e_{\text {sCO2 }}=((-8182)-(-8949))-288,7(5,844-4,826)=473,1 \mathrm{~kJ} / \mathrm{kg} \\
& e_{\text {SH } 2 O}=((-12040)-(-13440))-288,7(12,36-10,41)=838 \mathrm{~kJ} / \mathrm{kg} \\
& e_{s N_{2}}=(597,2-(-9,829))-288,7(7,828-6,799)=310 \mathrm{~kJ} / \mathrm{kg} \\
& e_{\text {so } 2}=(551-(-8,713))-288,7(7,323-6,374)=285,8 \mathrm{~kJ} / \mathrm{kg} \\
& e_{s h p}=(3377-65,34)-288,7(6,397-0,2323)=1532 \mathrm{~kJ} / \mathrm{kg}
\end{aligned}
$$

Matéria prima:

$$
\begin{aligned}
& E_{m}=\sum \dot{m}_{i} e_{e i}\left(\mathrm{i}=\mathrm{hp}, \mathrm{am}, \mathrm{CO}_{2}, \mathrm{H}_{2} \mathrm{O}, \mathrm{N}_{2}, \mathrm{O}_{2}\right) \\
& E_{m}=172,6 \cdot 309,6+28,05 \cdot 486,7+22,97 \cdot 864,1+197,8 \cdot 324,1+19,29 \cdot 298,8+88,24 \cdot 1476=287060 \mathrm{~kW}
\end{aligned}
$$

Produtos:

$$
\begin{aligned}
& E_{p}=\dot{m}_{s h p} e_{S h p} \\
& E_{p}=88,24 \cdot 1532=135183 \mathrm{~kW}
\end{aligned}
$$

Perdas:

$$
\begin{aligned}
& E_{l}=\sum \dot{m}_{i} e_{s i},\left(i=a m, C_{2}, H_{2} O, N_{2}, O_{2}\right) \\
& E_{l}=172,6 \cdot 295,9+28,05 \cdot 473,1+22,97 \cdot 838+197,8 \cdot 310+19,29 \cdot 285,8=150437 \mathrm{~kW}
\end{aligned}
$$

Exergia dissipada:

$$
\begin{aligned}
& \dot{E}_{d}=\dot{E}_{m}-\left(\dot{E}_{p}+\dot{E}_{l}\right) \\
& \dot{E}_{d}=287060-(135183+150437)=1440 \mathrm{~kW}
\end{aligned}
$$

Eficiência exergética:

$$
\begin{aligned}
& \varepsilon=\frac{\dot{E}_{p}}{\dot{E}_{m}} \\
& \varepsilon=\frac{135183}{287060}=0,47
\end{aligned}
$$


Eficiência exergética maior:

$$
\begin{aligned}
& \varepsilon^{+}=\frac{\dot{E}_{p}+\dot{E}_{l}}{\dot{E}_{m}} \\
& \varepsilon^{+}=\frac{135183+150437}{287060}=0,99
\end{aligned}
$$

4.4.1.3 Volume de controle entre os pontos 2 e 3 (Superaquecedor MP $-2^{\circ}$ passo)

\subsection{Condições na entrada}

Temperatura na entrada:

$T_{e_{g}}=590,1^{\circ} \mathrm{C}$

$T_{\text {emp }}=408,1^{\circ} \mathrm{C}$

Pressão na entrada:

$$
\begin{aligned}
& p_{e_{m p}}=3412 \mathrm{kPa} \\
& p_{e_{g}}=\frac{103,3}{(1-0,03)^{4} \cdot(1-0,03)^{3} \cdot(1-0,03)^{8}}=163,2 \mathrm{kPa}
\end{aligned}
$$

Entalpia na entrada:

Valores das entalpias obtidos pelo programa EES já reduzidos pelo coeficiente igual a 0,02.

$$
\begin{aligned}
& h_{\text {eam }}=874,2 \mathrm{~kJ} / \mathrm{kg} \\
& h_{\text {eсо } 2}=-8182 \mathrm{~kJ} / \mathrm{kg} \\
& h_{\text {eн } 20}=-12040 \mathrm{~kJ} / \mathrm{kg} \\
& h_{\text {eN } 2}=597,2 \mathrm{~kJ} / \mathrm{kg} \\
& h_{\text {eо } 2}=551 \mathrm{~kJ} / \mathrm{kg} \\
& h_{\text {emp }}=3242 \mathrm{~kJ} / \mathrm{kg}
\end{aligned}
$$

Entropia na entrada:

$$
\begin{aligned}
& S_{\text {eam }}=6,661 \mathrm{~kJ} / \mathrm{kgK} \\
& S_{\text {eсо } 2}=5,844 \mathrm{~kJ} / \mathrm{kgK} \\
& S_{\text {eH } 20}=12,36 \mathrm{~kJ} / \mathrm{kgK} \\
& S_{e^{2} 2}=7,828 \mathrm{~kJ} / \mathrm{kgK} \\
& S_{e о 2}=7,323 \mathrm{~kJ} / \mathrm{kgK} \\
& S_{\text {emp }}=6,881 \mathrm{~kJ} / \mathrm{kgK}
\end{aligned}
$$

\subsection{Equações de balanço}

Balanço de Massa considerado.

Valor da vazão mássica associada a cada substância presente no volume de controle

$$
\begin{aligned}
& \dot{m}_{\text {eam }}=172,6 \mathrm{~kg} / \mathrm{s} \\
& \dot{m}_{\text {eСO2 }}=28,05 \mathrm{~kg} / \mathrm{s} \\
& \dot{m}_{\mathrm{HH}^{2 O} \mathrm{O}}=22,97 \mathrm{~kg} / \mathrm{s} \\
& \dot{m}_{\mathrm{eN}^{2}}=197,8 \mathrm{~kg} / \mathrm{s}
\end{aligned}
$$




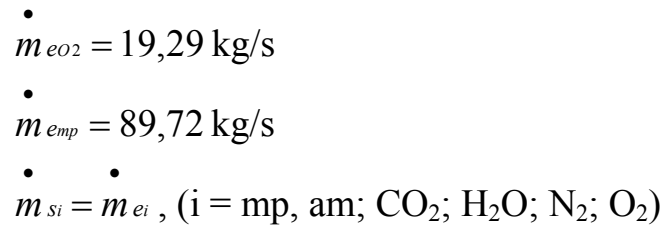

Balanço de energia

Da mesma forma como no balanço de massa, não será necessário determinar nenhuma variável pelo balanço de energia para este volume de controle.

\subsection{Condições na saída}

Temperatura na saída:

$T_{s_{g}}=557,4^{\circ} \mathrm{C}$

(Gases)

$T_{\text {smp }}=538,7^{\circ} \mathrm{C}$

(Vapor)

Pressão na saída:

$$
\begin{aligned}
& p_{\text {smp }}=3312 \mathrm{kPa} \\
& p_{s_{s}}=\frac{103,3}{(1-0,03)^{4} \cdot(1-0,03)^{3} \cdot(1-0,03)^{7}}=158,3 \mathrm{kPa}
\end{aligned}
$$

Entalpia na saída:

$$
\begin{aligned}
& h_{\text {sam }}=855,7 \mathrm{~kJ} / \mathrm{kg} \\
& h_{\text {sCO2 }}=-8388 \mathrm{~kJ} / \mathrm{kg} \\
& h_{\text {sH } 2 O}=-12357 \mathrm{~kJ} / \mathrm{kg} \\
& h_{\text {sN } 2}=572,2 \mathrm{~kJ} / \mathrm{kg} \\
& h_{\text {so } 2}=527,5 \mathrm{~kJ} / \mathrm{kg} \\
& h_{\text {smp }}=3541 \mathrm{~kJ} / \mathrm{kg}
\end{aligned}
$$

Entropia na saída:

$$
\begin{aligned}
& S_{\text {sam }}=6,627 \mathrm{~kJ} / \mathrm{kgK} \\
& s_{s \mathrm{CO}_{2}}=5,804 \mathrm{~kJ} / \mathrm{kgK} \\
& S_{S \mathrm{H} 2 \mathrm{O}}=12,29 \mathrm{~kJ} / \mathrm{kgK} \\
& S_{S_{N 2}}=7,793 \mathrm{~kJ} / \mathrm{kgK} \\
& s_{s o 2}=7,29 \mathrm{~kJ} / \mathrm{kgK} \\
& s_{s m p}=7,295 \mathrm{~kJ} / \mathrm{kgK}
\end{aligned}
$$

\subsection{Análise exergética}

Exergia dos fluxos de massa na entrada:

$$
\begin{aligned}
& e_{e_{i}}=\left(h_{e_{i}}-h_{0_{i}}\right)-T_{0}\left(s_{e_{i}}-s 0_{i}\right),\left(\mathrm{i}=\mathrm{mp}, \mathrm{am}, \mathrm{CO}_{2}, \mathrm{H}_{2} \mathrm{O}, \mathrm{N}_{2}, \mathrm{O}_{2}\right) \\
& e_{\text {eam }}=(874,2-289,1)-288,7(6,661-5,659)=295,9 \mathrm{~kJ} / \mathrm{kg} \\
& e_{\text {есог }}=((-8182)-(-8949))-288,7(5,844-4,826)=473,1 \mathrm{~kJ} / \mathrm{kg} \\
& e_{\text {енго }}=((-12040)-(-13440))-288,7(12,36-10,41)=838 \mathrm{~kJ} / \mathrm{kg} \\
& e_{\text {еN2 } 2}=(597,2-(-9,829))-288,7(7,828-6,799)=310 \mathrm{~kJ} / \mathrm{kg} \\
& e_{\text {eо } 2}=(551-(-8,713))-288,7(7,323-6,374)=285,8 \mathrm{~kJ} / \mathrm{kg} \\
& e_{\text {етр }}=(3242-65,34)-288,7(6,881-0,2323)=1257 \mathrm{~kJ} / \mathrm{kg}
\end{aligned}
$$

Exergia dos fluxos de massa na saída: 


$$
\begin{aligned}
& e_{s i}=\left(h_{s i}-h_{0 i}\right)-T_{0}\left(s_{s i}-s 0_{i}\right),\left(\mathrm{i}=\mathrm{hp}, \text { am, } \mathrm{CO}_{2}, \mathrm{H}_{2} \mathrm{O}, \mathrm{N}_{2}, \mathrm{O}_{2}\right) \\
& e_{\text {sam }}=(855,7-289,1)-288,7(6,627-5,659)=287,3 \mathrm{~kJ} / \mathrm{kg} \\
& e_{\text {sCO2 }}=((-8388)-(-8949))-288,7(5,804-4,826)=278,9 \mathrm{~kJ} / \mathrm{kg} \\
& e_{\text {SH } 2 O}=((-12357)-(-13440))-288,7(12,29-10,41)=541 \mathrm{~kJ} / \mathrm{kg} \\
& e_{S_{N} 2}=(572,2-(-9,829))-288,7(7,793-6,799)=295,1 \mathrm{~kJ} / \mathrm{kg} \\
& e_{s_{2} 2}=(527,5-(-8,713))-288,7(7,29-6,374)=271,8 \mathrm{~kJ} / \mathrm{kg} \\
& e_{s h p}=(3541-65,34)-288,7(7,295-0,2323)=1436 \mathrm{~kJ} / \mathrm{kg}
\end{aligned}
$$

Matéria prima:

$$
\begin{aligned}
& E_{m}=\sum \dot{m}_{i} e_{e_{i}}\left(\mathrm{i}=\mathrm{hp}, \mathrm{am}, \mathrm{CO}_{2}, \mathrm{H}_{2} \mathrm{O}, \mathrm{N}_{2}, \mathrm{O}_{2}\right) \\
& E_{m}=172,6 \cdot 295,9+28,05 \cdot 473,1+22,97 \cdot 838+197,8 \cdot 310+19,29 \cdot 285,8+89,72 \cdot 1257=263253 \mathrm{~kW}
\end{aligned}
$$

Produtos:

$$
\begin{aligned}
& \dot{E}_{p}=\dot{m}_{s h p} e_{S h p} \\
& E_{p}=89,72 \cdot 1436=128862 \mathrm{~kW}
\end{aligned}
$$

Perdas:

$$
\begin{aligned}
& E_{l}=\sum \dot{m}_{i} e_{s i},\left(i=a m, \mathrm{CO}_{2}, \mathrm{H}_{2} \mathrm{O}, \mathrm{N}_{2}, \mathrm{O}_{2}\right) \\
& E_{l}=172,6 \cdot 287,3+28,05 \cdot 278,9+22,97 \cdot 541+197,8 \cdot 295,1+19,29 \cdot 271,8=133458 \mathrm{~kW}
\end{aligned}
$$

Exergia dissipada:

$$
\begin{aligned}
& \dot{E}_{d}=\dot{E}_{m}-\left(\dot{E}_{p}+\dot{E}_{l}\right) \\
& \dot{E}_{d}=263253-(128862+133458)=933 \mathrm{~kW}
\end{aligned}
$$

Eficiência exergética:

$$
\begin{aligned}
& \varepsilon=\frac{\dot{E}_{p}}{\dot{E}_{m}} \\
& \varepsilon=\frac{128862}{263253}=0,49
\end{aligned}
$$

Eficiência exergética maior:

$$
\begin{aligned}
\varepsilon^{+} & =\frac{\dot{E}_{p}+\dot{E}_{l}}{\dot{E}_{m}} \\
\varepsilon^{+} & =\frac{128862+133458}{263253}=0,99
\end{aligned}
$$

4.4.1.4 Volume de controle entre os pontos 3 e 4 (Superaquecedor $\mathrm{HP}-2^{\circ}$ passo)

Neste volume de controle, ocorre a queima suplementar. Como há a presença de um processo reativo, será acrescentada a equação e a respectiva energia incluída pelo calor liberado. 
Considera-se que o combustível fornecido irá reagir com o ar de mistura que foi inserido antes da entrada na turbina a gás.

\subsection{Condições na entrada}

Para identificar o fluxo de combustível será atribuído o índice $c b$.

Temperatura na entrada:

$T_{e_{g}}=557,4^{\circ} \mathrm{C}$

(Gases)

$T_{e c b}=129,4^{\circ} \mathrm{C}$

(Combustível)

$T_{e h p}=440,9^{\circ} \mathrm{C}$

(Vapor)

Pressão na entrada:

$$
\begin{aligned}
& p_{\text {ehp }}=16678 \mathrm{kPa} \\
& p_{\text {ecb }}=3172 \mathrm{kPa} \\
& p_{e_{g}}=\frac{103,3}{(1-0,03)^{4} \cdot(1-0,03)^{3} \cdot(1-0,03)^{7}}=158,3 \mathrm{kPa}
\end{aligned}
$$

Entalpia na entrada:

$$
\begin{aligned}
& h_{\text {eam }}=838,6 \mathrm{~kJ} / \mathrm{kg} \\
& h_{\text {eсо } 2}=-8220 \mathrm{~kJ} / \mathrm{kg} \\
& h_{\text {eH } 20}=-12110 \mathrm{~kJ} / \mathrm{kg} \\
& h_{\text {eN2 } 2}=560,8 \mathrm{~kJ} / \mathrm{kg} \\
& h_{\text {eо } 2}=516,9 \mathrm{~kJ} / \mathrm{kg} \\
& h_{\text {ecb }}=-4418 \mathrm{~kJ} / \mathrm{kg} \\
& h_{\text {ehp }}=3094 \mathrm{~kJ} / \mathrm{kg}
\end{aligned}
$$

Entropia na entrada:

$$
\begin{aligned}
& \text { Seam }=6,627 \mathrm{~kJ} / \mathrm{kgK} \\
& \text { Seco2 }=5,804 \mathrm{~kJ} / \mathrm{kgK} \\
& \text { Sен } 2 \text { O }=12,29 \mathrm{~kJ} / \mathrm{kgK} \\
& S_{e_{N} 2}=7,793 \mathrm{~kJ} / \mathrm{kgK} \\
& S_{\text {eo } 2}=7,29 \mathrm{~kJ} / \mathrm{kgK} \\
& \mathrm{Sec}_{b}=10,53 \mathrm{~kJ} / \mathrm{kgK} \\
& S_{\text {ehp }}=6,014 \mathrm{~kJ} / \mathrm{kgK}
\end{aligned}
$$

\subsection{Equações de balanço}

Balanço de Massa:

Nesse caso o balanço de massa será determinado pela quantidade de moles envolvida no processo de reação. Primeiramente será informada a massa das substâncias que entram no volume de controle.

$$
\begin{aligned}
& \dot{m}_{\text {eam }}=172,6 \mathrm{~kg} / \mathrm{s} \\
& \dot{m}_{e \mathrm{CO}_{2}}=28,05 \mathrm{~kg} / \mathrm{s} \\
& \dot{m}_{\text {eH } 2 O}=22,97 \mathrm{~kg} / \mathrm{s} \\
& \dot{m}_{\text {eN } 2}=197,8 \mathrm{~kg} / \mathrm{s}
\end{aligned}
$$




$$
\begin{aligned}
& \dot{m}_{e O 2}=19,29 \mathrm{~kg} / \mathrm{s} \\
& \dot{m}_{e c b}=2,02 \mathrm{~kg} / \mathrm{s} \\
& \dot{m}_{e h p}=88,24 \mathrm{~kg} / \mathrm{s}
\end{aligned}
$$

Processo de combustão

Da mesma forma desenvolvida para a combustão no combustor, será utilizada a equação para a reação do metano com o ar proveniente do processo de mistura.

Equação para a combustão do metano:

$$
\mathrm{CH}_{4}+\lambda \cdot 2 \cdot \mathrm{O}_{2}+\lambda \cdot 2 \cdot 3,76 \cdot \mathrm{N}_{2} \rightarrow \mathrm{CO}_{2}+2 \cdot \mathrm{H}_{2} \mathrm{O}+\lambda \cdot 2 \cdot 3,76 \cdot \mathrm{N}_{2}+(\lambda-1) \cdot 2 \cdot \mathrm{O}_{2}
$$

A quantidade de moles associada aos gases será calculada a seguir

Moles de Ar:

$$
\dot{m o l} \text { ar }=\frac{172,6}{28,97}=5,959 \mathrm{kmol} / \mathrm{s}
$$

Moles de Combustível:

$$
\dot{m o l} l_{c b}=\frac{2,02}{16,04}=0,1259 \mathrm{kmol} / \mathrm{s}
$$

Assim, pode-se obter o valor de $\lambda$

$$
\lambda=\frac{172,6}{(1+3,76) \cdot 2 \cdot 0,1259 \cdot 28,97}=4,971
$$

Finalmente pode-se obter a massa de cada produto da combustão

Massa de $\mathrm{CO}_{2}$ :

$\dot{m}_{\mathrm{CO}_{2}}=1 \cdot 0,1259 \cdot 44,01=5,54 \mathrm{~kg} / \mathrm{s}$

$$
\left(\mathrm{M}_{\mathrm{CO} 2}=44,01\right)
$$

Massa de $\mathrm{H}_{2} \mathrm{O}$ :

$$
\dot{m}_{\mathrm{H}_{2} \mathrm{O}}=2 \cdot 0,1259 \cdot 18,02=4,537 \mathrm{~kg} / \mathrm{s}
$$

Massa de $\mathrm{N}_{2}$ :

$$
\dot{m}_{N_{2}}=3,76 \cdot 4,971 \cdot 2 \cdot 0,1259 \cdot 28,01=131,8 \mathrm{~kg} / \mathrm{s}
$$

Massa de $\mathrm{O}_{2}$ :

$$
\dot{m}_{O_{2}}=(4,971-1) \cdot 2 \cdot 0,1259 \cdot 32=32 \mathrm{~kg} / \mathrm{s}
$$

$$
\left(\mathrm{M}_{\mathrm{O} 2}=32\right)
$$

A quantidade de massa que sai do volume de controle é dada pela soma dos fluxos na entrada mais o acréscimo associado ao processo reativo entre o combustível e o ar de mistura. Assim têm-se os seguintes fluxos na saída do volume de controle considerado 


$$
\begin{aligned}
& \dot{m}_{S \mathrm{SO}_{2}}=28,05+5,541=33,59 \mathrm{~kg} / \mathrm{s} \\
& \dot{m}_{S \mathrm{~S}_{2 O} O}=22,97+4,537=27,51 \mathrm{~kg} / \mathrm{s} \\
& \dot{m}_{S{ }^{2} 2}=197,8+131,8=329,6 \mathrm{~kg} / \mathrm{s} \\
& \dot{m}_{S O 2}=19,29+32=51,29 \mathrm{~kg} / \mathrm{s}
\end{aligned}
$$

Balanço de energia

A seguir dera descrita a equação para o balanço de energia em relação as substâncias envolvidas no processo de reação.

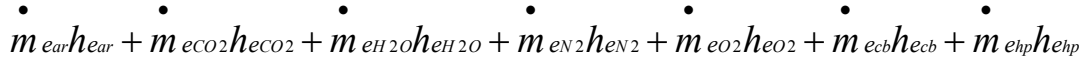

$$
\begin{aligned}
& = \\
& \dot{m}_{S \mathrm{CO} 2} h_{S \mathrm{CO} 2}+\dot{m}_{S \mathrm{H} 2 \mathrm{OO}} h_{S \mathrm{H} 2 \mathrm{O}}+\dot{m}_{S \mathrm{~S} 2} h_{S \mathrm{~N} 2}+\dot{m}_{S O 2} h_{S O 2}+\dot{m}_{S h p} h_{S h p}
\end{aligned}
$$

\subsection{Condições na saída}

Temperatura na saída:

$$
T_{s g}=675,6^{\circ} \mathrm{C}
$$

Pode-se observar que a temperatura dos gases na saída e maior do que a temperatura na entrada. Isso ocorre em conseqüência da energia acrescentada pelo processo de reação.

$$
T_{s h p}=499,6^{\circ} \mathrm{C}
$$

Pressão na saída:

$$
\begin{aligned}
& p_{s h p}=16499 \mathrm{kPa} \\
& p_{s g}=\frac{103,3}{(1-0,03)^{4} \cdot(1-0,03)^{3} \cdot(1-0,03)^{6}}=153,6 \mathrm{kPa}
\end{aligned}
$$

Entalpia na saída:

$$
\begin{aligned}
& h_{\text {SCO2 }}=-8082 \mathrm{~kJ} / \mathrm{kg} \\
& h_{\text {SH } 20}=-11853 \mathrm{~kJ} / \mathrm{kg} \\
& h_{\text {SN } 2}=693,6 \mathrm{~kJ} / \mathrm{kg} \\
& h_{\text {sO2 }}=640,8 \mathrm{~kJ} / \mathrm{kg} \\
& h_{\text {shp }}=3288 \mathrm{~kJ} / \mathrm{kg}
\end{aligned}
$$

Entropia na saída:

$$
\begin{aligned}
& S_{S \mathrm{C}_{2}}=5,97 \mathrm{~kJ} / \mathrm{kgK} \\
& S_{S H 2 O}=12,6 \mathrm{~kJ} / \mathrm{kgK} \\
& s_{s 2}=7,954 \mathrm{~kJ} / \mathrm{kgK} \\
& s_{\text {so } 2}=7,44 \mathrm{~kJ} / \mathrm{kgK} \\
& S_{s h p}=6,279 \mathrm{~kJ} / \mathrm{kgK}
\end{aligned}
$$

\subsection{Análise exergética}

Exergia dos fluxos de massa na entrada:

$$
\begin{aligned}
& e_{e i}=\left(h_{e_{i}}-h_{0_{i}}\right)-T_{0}\left(s_{e i}-s 0_{i}\right),\left(\mathrm{i}=\mathrm{cb}, \mathrm{hp}, \mathrm{am}, \mathrm{CO}_{2}, \mathrm{H}_{2} \mathrm{O}, \mathrm{N}_{2}, \mathrm{O}_{2}\right) \\
& e_{e a m}=(838,6-289,1)-288,7(6,627-5,659)=270,2 \mathrm{~kJ} / \mathrm{kg}
\end{aligned}
$$


$e_{e c b}=((-4418)-(-4688))-288,7(10,53-11,53)+46518=47076 \mathrm{~kJ} / \mathrm{kg}$

$e_{\text {eсо } 2}=((-8220)-(-8949))-288,7(5,804-4,826)=446,7 \mathrm{~kJ} / \mathrm{kg}$

$e_{\text {енго }}=((-12110)-(-13440))-288,7(12,29-10,41)=788,1 \mathrm{~kJ} / \mathrm{kg}$

$e_{\text {eN2 }}=(560,8-(-9,829))-288,7(7,793-6,799)=283,7 \mathrm{~kJ} / \mathrm{kg}$

$e_{\text {eо } 2}=(516,9-(-8,713))-288,7(7,29-6,374)=261,3 \mathrm{~kJ} / \mathrm{kg}$

$e_{e h p}=(3094-65,34)-288,7(6,014-0,2323)=1359 \mathrm{~kJ} / \mathrm{kg}$

Exergia dos fluxos de massa na saída:

$e_{s i}=\left(h_{s i}-h_{0 i}\right)-T_{0}\left(s_{s i}-s_{0 i}\right),\left(i=h p, \mathrm{CO}_{2}, \mathrm{H}_{2} \mathrm{O}, \mathrm{N}_{2}, \mathrm{O}_{2}\right)$

(Eq. 2.14)

$e_{\text {sCO2 }}=((-8082)-(-8949))-288,7(5,97-4,826)=537,8 \mathrm{~kJ} / \mathrm{kg}$

$e_{\text {SH } 2 O}=((-11853)-(-13440))-288,7(12,6-10,41)=956 \mathrm{~kJ} / \mathrm{kg}$

$e_{S N 2}=(693,6-(-9,829))-288,7(7,954-6,799)=369,8 \mathrm{~kJ} / \mathrm{kg}$

$e_{\text {so } 2}=(640,8-(-8,713))-288,7(7,44-6,374)=341,8 \mathrm{~kJ} / \mathrm{kg}$

$e_{\text {sh }}=(3288-65,34)-288,7(6,279-0,2323)=1477 \mathrm{~kJ} / \mathrm{kg}$

Matéria prima:

$E_{m}=\sum \dot{m}_{i} e_{e i}\left(\mathrm{i}=\mathrm{hp}, \mathrm{cb}, \mathrm{am}, \mathrm{CO}_{2}, \mathrm{H}_{2} \mathrm{O}, \mathrm{N}_{2}, \mathrm{O}_{2}\right)$

$E_{m}=172,6 \cdot 270,2+28,05 \cdot 446,7+22,97 \cdot 788,1+197,8 \cdot 283,7+19,29 \cdot 261,3$

$+88,24 \cdot 1359+2,02 \cdot 47076=353452 \mathrm{~kW}$

Produtos:

$E_{p}=\dot{m}_{\text {shp }} e_{s h p}$

$E_{p}=88,24 \cdot 1477=130291 \mathrm{~kW}$

(Eq. 3.76)

Perdas:

$E_{l}=\sum \dot{m}_{i} e_{s i},\left(i=\mathrm{CO}_{2}, \mathrm{H}_{2} \mathrm{O}, \mathrm{N}_{2}, \mathrm{O}_{2}\right)$

(Eq. 3.77)

$E_{l}=33,59 \cdot 537,8+27,51 \cdot 956+329,6 \cdot 369,8=183811 \mathrm{~kW}$

Exergia dissipada:

$\dot{E}_{d}=\dot{E}_{m}-\left(\dot{E}_{p}+\dot{E}_{l}\right)$

$\dot{E}_{d}=353452-(130291+183811)=39350 \mathrm{~kW}$

Eficiência exergética:

$$
\begin{aligned}
& \varepsilon=\frac{\dot{E}_{p}}{\dot{E}_{m}} \\
& \varepsilon=\frac{130291}{353459}=0,37
\end{aligned}
$$

Eficiência exergética maior: 


$$
\begin{aligned}
\varepsilon^{+} & =\frac{\dot{E}_{p}+\dot{E}_{l}}{\dot{E}_{m}} \\
\varepsilon^{+} & =\frac{130291+183811}{353452}=0,89
\end{aligned}
$$

4.4.1.5 Volume de controle entre os pontos 4 e 5 (Superaquecedor MP $-1^{\circ}$ passo)

\subsection{Condições na entrada}

Temperatura na entrada:

$T_{e g}=675,6^{\circ} \mathrm{C}$

(Gases)

$T_{\text {emp }}=322,7^{\circ} \mathrm{C}$

Pressão na entrada:

$$
\begin{aligned}
& p_{e_{m p}}=3511 \mathrm{kPa} \\
& p_{e}=\frac{103,3}{(1-0,03)^{4} \cdot(1-0,03)^{3} \cdot(1-0,03)^{6}}=153,6 \mathrm{kPa}
\end{aligned}
$$

Entalpia na entrada:

Valores das entalpias obtidos pelo programa EES já reduzidos pelo coeficiente igual a 0,02.

$$
\begin{aligned}
& h_{\text {eсо } 2}=-8082 \mathrm{~kJ} / \mathrm{kg} \\
& h_{\text {eн } 20}=-11853 \mathrm{~kJ} / \mathrm{kg} \\
& h_{\text {eN2 }}=693,6 \mathrm{~kJ} / \mathrm{kg} \\
& h_{\text {eо } 2}=640,8 \mathrm{~kJ} / \mathrm{kg} \\
& h_{\text {emp }}=3035 \mathrm{~kJ} / \mathrm{kg}
\end{aligned}
$$

Entropia na entrada:

$$
\begin{aligned}
& \text { Sесо } 2=5,97 \mathrm{~kJ} / \mathrm{kgK} \\
& S_{\text {eн } 20}=12,6 \mathrm{~kJ} / \mathrm{kgK} \\
& S_{\text {eN } 2}=7,954 \mathrm{~kJ} / \mathrm{kgK} \\
& S_{\text {eо } 2}=7,44 \mathrm{~kJ} / \mathrm{kgK} \\
& S_{\text {emp }}=6,544 \mathrm{~kJ} / \mathrm{kgK}
\end{aligned}
$$

\subsection{Equações de balanço}

\section{Balanço de Massa} considerado.

Valor da vazão mássica associada a cada substância presente no volume de controle

$$
\begin{aligned}
& \dot{m}_{\text {eСO2 }}=33,59 \mathrm{~kg} / \mathrm{s} \\
& \dot{m}_{\text {eH } 2 O}=27,51 \mathrm{~kg} / \mathrm{s} \\
& \dot{m}_{e^{N 2}}=329,6 \mathrm{~kg} / \mathrm{s} \\
& \dot{m}_{e O 22}=51,29 \mathrm{~kg} / \mathrm{s} \\
& \dot{m}_{e^{m p}}=89,72 \mathrm{~kg} / \mathrm{s} \\
& \dot{m}_{S i}=\dot{m}_{e i},\left(\mathrm{i}=\mathrm{mp}, \mathrm{CO}_{2} ; \mathrm{H}_{2} \mathrm{O} ; \mathrm{N}_{2} ; \mathrm{O}_{2}\right)
\end{aligned}
$$


Balanço de energia

Da mesma forma como no balanço de massa, não será necessário determinar nenhuma variável pelo balanço de energia para este volume de controle.

\subsection{Condições na saída}

Temperatura na saída:

$T_{s_{g}}=640,9^{\circ} \mathrm{C}$

(Gases)

$T_{s m p}=408,1^{\circ} \mathrm{C}$

(Vapor)

Pressão na saída:

$p_{s m p}=3412 \mathrm{kPa}$

$p_{s g}=\frac{103,3}{(1-0,03)^{4} \cdot(1-0,03)^{3} \cdot(1-0,03)^{5}}=149 \mathrm{kPa}$

Entalpia na saída:

$$
\begin{aligned}
& h_{\text {sCO2 }}=-8123 \mathrm{~kJ} / \mathrm{kg} \\
& h_{\text {sH } 20}=-11929 \mathrm{~kJ} / \mathrm{kg} \\
& h_{\text {N } 2^{2}}=654,4 \mathrm{~kJ} / \mathrm{kg} \\
& h_{s O_{2}}=604,2 \mathrm{~kJ} / \mathrm{kg} \\
& h_{\text {smp }}=3242 \mathrm{~kJ} / \mathrm{kg}
\end{aligned}
$$

Entropia na saída:

$$
\begin{aligned}
& S_{s \mathrm{CO}_{2}}=5,93 \mathrm{~kJ} / \mathrm{kgK} \\
& S_{S H 2 O}=12,53 \mathrm{~kJ} / \mathrm{kgK} \\
& S_{S N 2}=7,92 \mathrm{~kJ} / \mathrm{kgK} \\
& s_{s o 2}=7,408 \mathrm{~kJ} / \mathrm{kgK} \\
& s_{s m p}=6,881 \mathrm{~kJ} / \mathrm{kgK}
\end{aligned}
$$

\subsection{Análise exergética}

Exergia dos fluxos de massa na entrada:

$$
\begin{aligned}
& e_{e i}=\left(h_{e_{i}}-h_{0_{i}}\right)-T_{0}\left(s_{e_{i}}-s 0_{i}\right),\left(i=m p, \mathrm{CO}_{2}, \mathrm{H}_{2} \mathrm{O}, \mathrm{N}_{2}, \mathrm{O}_{2}\right) \\
& e_{e \text { со } 2}=((-8082)-(-8949))-288,7(5,97-4,826)=537,8 \mathrm{~kJ} / \mathrm{kg} \\
& e_{\text {ен } 2 \mathrm{O}}=((-11853)-(-13440))-288,7(12,6-10,41)=956 \mathrm{~kJ} / \mathrm{kg} \\
& e_{e \mathrm{~N}_{2}}=(693,6-(-9,829))-288,7(7,954-6,799)=369,8 \mathrm{~kJ} / \mathrm{kg} \\
& e_{e о 2}=(640,8-(-8,713))-288,7(7,44-6,374)=341,8 \mathrm{~kJ} / \mathrm{kg} \\
& e_{\text {emp }}=(3035-65,34)-288,7(6,544-0,2323)=1148 \mathrm{~kJ} / \mathrm{kg}
\end{aligned}
$$

Exergia dos fluxos de massa na saída:

$$
\begin{aligned}
& e_{s i}=\left(h_{s i}-h_{0}\right)-T 0\left(s_{s i}-s_{0}\right),\left(i=m p, \mathrm{CO}_{2}, \mathrm{H}_{2} \mathrm{O}, N_{2}, \mathrm{O}_{2}\right) \\
& e_{s O_{2}}=((-8123)-(-8949))-288,7(5,93-4,826)=508 \mathrm{~kJ} / \mathrm{kg} \\
& e_{s H 2 O}=((-11929)-(-13440))-288,7(12,53-10,41)=899,7 \mathrm{~kJ} / \mathrm{kg} \\
& e_{s N^{2}}=(654,4-(-9,829))-288,7(3,792-6,799)=340,4 \mathrm{~kJ} / \mathrm{kg} \\
& e_{s O 2}=(604,2-(-8,713))-288,7(7,408-6,374)=314,5 \mathrm{~kJ} / \mathrm{kg} \\
& e_{S m p}=(3242-65,34)-288,7(6,881-0,2323)=1257 \mathrm{~kJ} / \mathrm{kg}
\end{aligned}
$$

Matéria prima: 
$E_{m}=\sum \dot{m}_{i} e_{e i}\left(i=m p, \mathrm{CO}_{2}, \mathrm{H}_{2} \mathrm{O}, \mathrm{N}_{2}, \mathrm{O}_{2}\right)$

$E_{m}=33,59 \cdot 537,8+27,51 \cdot 956+329,6 \cdot 369,8+51,29 \cdot 341,8+88,72 \cdot 1148=286786 \mathrm{~kW}$

Produtos:

$E_{p}=\dot{m}_{\text {smp }} e_{s m p}$

$E_{p}=89,72 \cdot 1257=112816 \mathrm{~kW}$

Perdas:

$E_{l}=\sum \dot{m}_{i} e_{s i},\left(i=\mathrm{CO}_{2}, \mathrm{H}_{2} \mathrm{O}, \mathrm{N}_{2}, \mathrm{O}_{2}\right)$

$E_{l}=33,59 \cdot 508+27,51 \cdot 899,7+329,6 \cdot 340,4+51,29 \cdot 314,5=170140 \mathrm{~kW}$

Exergia dissipada:

$\dot{E}_{d}=\dot{E}_{m}-\left(\dot{E}_{p}+\dot{E}_{l}\right)$

$\dot{E}_{d}=286786-(112816+170140)=3829 \mathrm{~kW}$

Eficiência exergética:

$$
\begin{aligned}
& \varepsilon=\frac{\dot{E}_{p}}{\dot{E}_{m}} \\
& \varepsilon=\frac{112816}{286786}=0,39
\end{aligned}
$$

Eficiência exergética maior:

$$
\begin{aligned}
\varepsilon^{+} & =\frac{\dot{E}_{p}+\dot{E}_{l}}{\dot{E}_{m}} \\
\varepsilon^{+} & =\frac{112816+170140}{286786}=0,98
\end{aligned}
$$

4.4.1.6 Volume de controle entre os pontos 5 e 6 (Superaquecedor $\mathrm{HP}-1^{\circ}$ passo)

\subsection{Condições na entrada}

Temperatura na entrada:

$T_{e g}=640,9^{\circ} \mathrm{C}$

$T_{\text {ehp }}=351,6^{\circ} \mathrm{C}$

Pressão na entrada:

$p_{e h p}=16858 \mathrm{kPa}$

$p_{e g}=\frac{103,3}{(1-0,03)^{4} \cdot(1-0,03)^{3} \cdot(1-0,03)^{5}}=149 \mathrm{kPa}$

Entalpia na entrada:

Valores das entalpias obtidos pelo programa EES já reduzidos pelo coeficiente igual a 0,02. 


$$
\begin{aligned}
& h_{\text {ен } 2 \mathrm{O}}=-11929 \mathrm{~kJ} / \mathrm{kg} \\
& h_{\text {eN } 2}=654,4 \mathrm{~kJ} / \mathrm{kg} \\
& h_{\text {eо } 2}=604,2 \mathrm{~kJ} / \mathrm{kg} \\
& h_{\text {ehp }}=2553 \mathrm{~kJ} / \mathrm{kg}
\end{aligned}
$$

Entropia na entrada:

$$
\begin{aligned}
& \text { Seсо }_{2}=5,93 \mathrm{~kJ} / \mathrm{kgK} \\
& \text { Sен } 20=12,53 \mathrm{~kJ} / \mathrm{kgK} \\
& S_{\text {eN } 2}=7,92 \mathrm{~kJ} / \mathrm{kgK} \\
& S_{\text {eо } 2}=7,408 \mathrm{~kJ} / \mathrm{kgK} \\
& S_{\text {ehp }}=5,189 \mathrm{~kJ} / \mathrm{kgK}
\end{aligned}
$$

\subsection{Equações de balanço}

\section{Balanço de Massa} considerado.

Valor da vazão mássica associada a cada substância presente no volume de controle

$$
\dot{m}_{e \mathrm{CO} 2}=33,59 \mathrm{~kg} / \mathrm{s}
$$$$
\dot{m}_{\text {eн } 20}=27,51 \mathrm{~kg} / \mathrm{s}
$$$$
\dot{m}_{e_{N 2}}=329,6 \mathrm{~kg} / \mathrm{s}
$$$$
\dot{m}_{e o 2}=51,29 \mathrm{~kg} / \mathrm{s}
$$$$
\dot{m}_{\text {ehp }}=88,24 \mathrm{~kg} / \mathrm{s}
$$$$
\dot{m}_{s i}=\dot{m}_{e i},\left(\mathrm{i}=\mathrm{hp}, \mathrm{CO}_{2} ; \mathrm{H}_{2} \mathrm{O} ; \mathrm{N}_{2} ; \mathrm{O}_{2}\right)
$$

Balanço de energia

Da mesma forma como no balanço de massa, não será necessário determinar nenhuma variável pelo balanço de energia para este volume de controle.

\subsection{Condições na saída}

Temperatura na saída:

$T_{s_{g}}=550,2^{\circ} \mathrm{C}$

$T_{s h p}=440,9^{\circ} \mathrm{C}$ (Vapor)

Pressão na saída:

$p_{s h p}=16678 \mathrm{kPa}$

$p_{s_{g}}=\frac{103,3}{(1-0,03)^{4} \cdot(1-0,03)^{3} \cdot(1-0,03)^{4}}=144,5 \mathrm{kPa}$

Entalpia na saída:

$$
\begin{aligned}
& h_{\text {SСO } 2}=-8229 \mathrm{~kJ} / \mathrm{kg} \\
& h_{\text {SH } 2 O}=-12126 \mathrm{~kJ} / \mathrm{kg} \\
& h_{\text {SN } 2}=552,8 \mathrm{~kJ} / \mathrm{kg} \\
& h_{\text {sO2 }}=509,4 \mathrm{~kJ} / \mathrm{kg} \\
& h_{\text {Shp }}=3094 \mathrm{~kJ} / \mathrm{kg}
\end{aligned}
$$


Entropia na saída:

$$
\begin{aligned}
& S_{s \mathrm{CO} 2}=5,811 \mathrm{~kJ} / \mathrm{kgK} \\
& s_{s H^{2 O}}=12,31 \mathrm{~kJ} / \mathrm{kgK} \\
& S_{S_{N} 2}=7,81 \mathrm{~kJ} / \mathrm{kgK} \\
& s_{s o 2}=7,304 \mathrm{~kJ} / \mathrm{kgK} \\
& s_{s h p}=6,014 \mathrm{~kJ} / \mathrm{kgK}
\end{aligned}
$$

\subsection{Análise exergética}

Exergia dos fluxos de massa na entrada:

$e_{e_{i}}=\left(h_{e_{i}}-h_{0 i}\right)-T_{0}\left(S_{e_{i}}-S_{0}\right),\left(i=h p, \mathrm{CO}_{2}, \mathrm{H}_{2} \mathrm{O}, \mathrm{N}_{2}, \mathrm{O}_{2}\right)$

$e_{\text {eсо2 }}=((-8123)-(-8949))-288,7(5,93-4,826)=508 \mathrm{~kJ} / \mathrm{kg}$

$e_{\text {ен } 2 \text { о }}=((-11929)-(-13440))-288,7(12,53-10,41)=899,7 \mathrm{~kJ} / \mathrm{kg}$

$e_{\text {eN } 2}=(654,4-(-9,829))-288,7(7,92-6,799)=340,4 \mathrm{~kJ} / \mathrm{kg}$

$e_{\text {eо } 2}=(604,2-(-8,713))-288,7(7,408-6,374)=314,5 \mathrm{~kJ} / \mathrm{kg}$

$e_{\text {ehp }}=(2553-65,34)-288,7(5,189-0,2323)=1056 \mathrm{~kJ} / \mathrm{kg}$

Exergia dos fluxos de massa na saída:

$e_{s i}=\left(h_{s i}-h_{0 i}\right)-T_{0}\left(s_{s i}-s_{0 i}\right),\left(i=h p, C O_{2}, H_{2} \mathrm{O}, N_{2}, O_{2}\right)$

$e_{\text {sCO2 }}=((-8229)-(-8949))-288,7(5,811-4,826)=436,3 \mathrm{~kJ} / \mathrm{kg}$

$e_{\text {SH } 2 O}=((-12126)-(-13440))-288,7(12,31-10,41)=766,1 \mathrm{~kJ} / \mathrm{kg}$

$e_{S_{N 2}}=(552,8-(-9,829))-288,7(7,81-6,799)=270,7 \mathrm{~kJ} / \mathrm{kg}$

$e_{\text {so } 2}=(509,4-(-8,713))-288,7(7,304-6,374)=249,6 \mathrm{~kJ} / \mathrm{kg}$

$e_{\text {shp }}=(3094-65,34)-288,7(6,014-0,2323)=1359 \mathrm{~kJ} / \mathrm{kg}$

Matéria prima:

$E_{m}=\sum \dot{m}_{i} e_{e i}\left(i=h p, \mathrm{CO}_{2}, \mathrm{H}_{2} \mathrm{O}, \mathrm{N}_{2}, \mathrm{O}_{2}\right)$

$E_{m}=33,59 \cdot 508+27,51 \cdot 899,7+329,6 \cdot 340,4+51,29 \cdot 314,5+88,24 \cdot 1056=263357 \mathrm{~kW}$

Produtos:

$$
\begin{aligned}
& E_{p}=\dot{m}_{s h p} e_{s h p} \\
& E_{p}=88,24 \cdot 1359=119945 \mathrm{~kW}
\end{aligned}
$$

Perdas:

$$
\begin{aligned}
& E_{l}=\sum \dot{m}_{i} e_{s i},\left(i=\mathrm{CO}_{2}, \mathrm{H}_{2} \mathrm{O}, \mathrm{N}_{2}, \mathrm{O}_{2}\right) \\
& E_{l}=33,59 \cdot 508+27,51 \cdot 899,7+329,6 \cdot 340,4+51,29 \cdot 314,5=137744 \mathrm{~kW}
\end{aligned}
$$

Exergia dissipada:

$$
\begin{aligned}
& \dot{E}_{d}=\dot{E}_{m}-\left(\dot{E}_{p}+\dot{E}_{l}\right) \\
& \dot{E}_{d}=263357-(119945+137744)=5668 \mathrm{~kW}
\end{aligned}
$$

Eficiência exergética: 
$\varepsilon=\frac{\dot{E}_{p}}{\dot{E}_{m}}$

$\varepsilon=\frac{119945}{263357}=0,45$

Eficiência exergética maior:

$$
\begin{aligned}
\varepsilon^{+} & =\frac{\dot{E}_{p}+\dot{E}_{l}}{\dot{E}_{m}} \\
\varepsilon^{+} & =\frac{119945+137744}{263357}=0,98
\end{aligned}
$$

4.4.1.7 Volume de controle entre os pontos 6 e 7 (Gerador de vapor HP)

\subsection{Condições na entrada}

Temperatura na entrada:

$T_{e_{g}}=550,2^{\circ} \mathrm{C}$

$T_{\text {ehp }}=322,4^{\circ} \mathrm{C}$

Pressão na entrada:

$$
\begin{aligned}
& p_{e h p}=170,73 \mathrm{kPa} \\
& p_{e_{g}}=\frac{103,3}{(1-0,03)^{4} \cdot(1-0,03)^{3} \cdot(1-0,03)^{4}}=144,5 \mathrm{kPa}
\end{aligned}
$$

Entalpia na entrada:

Valores das entalpias obtidos pelo programa EES já reduzidos pelo coeficiente igual a 0,02.

$$
\begin{aligned}
& h_{\text {есо } 2}=-8229 \mathrm{~kJ} / \mathrm{kg} \\
& h_{\text {ен } 20}=-12126 \mathrm{~kJ} / \mathrm{kg} \\
& h_{\text {eN } 2}=552,8 \mathrm{~kJ} / \mathrm{kg} \\
& h_{\text {eо } 2}=509,4 \mathrm{~kJ} / \mathrm{kg} \\
& h_{\text {ehp }}=1464 \mathrm{~kJ} / \mathrm{kg}
\end{aligned}
$$

Entropia na entrada:

$$
\begin{aligned}
& S_{\text {eco }}=5,811 \mathrm{~kJ} / \mathrm{kgK} \\
& \text { Sен } 2 \text { о }=12,31 \mathrm{~kJ} / \mathrm{kgK} \\
& S_{\text {eN } 2}=7,81 \mathrm{~kJ} / \mathrm{kgK} \\
& S_{\text {eo } 2}=7,304 \mathrm{~kJ} / \mathrm{kgK} \\
& S_{\text {ehp }}=3,438 \mathrm{~kJ} / \mathrm{kgK}
\end{aligned}
$$

\subsection{Equações de balanço}

\section{Balanço de Massa} considerado.

Valor da vazão mássica associada a cada substância presente no volume de controle $m_{\text {eco } 2}=33,59 \mathrm{~kg} / \mathrm{s}$ 


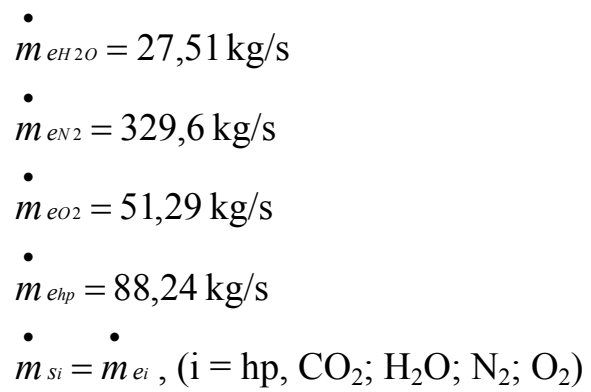

(Eq. 3.66 e 3.67$)$

Balanço de energia

Da mesma forma como no balanço de massa, não será necessário determinar nenhuma variável pelo balanço de energia para este volume de controle.

\subsection{Condições na saída}

Temperatura na saída:

$T_{s_{g}}=361,3^{\circ} \mathrm{C}$

(Gases)

$T_{s h p}=351,6^{\circ} \mathrm{C}$

(Vapor)

Pressão na saída:

$p_{s h p}=16858 \mathrm{kPa}$

$p_{s_{g}}=\frac{103,3}{(1-0,03)^{4} \cdot(1-0,03)^{2} \cdot(1-0,03)^{4}}=140,2 \mathrm{kPa}$

Entalpia na saída:

$$
\begin{aligned}
& h_{\text {SCO2 }}=-8439 \mathrm{~kJ} / \mathrm{kg} \\
& h_{\text {SH } 2 \mathrm{O}}=-12515 \mathrm{~kJ} / \mathrm{kg} \\
& h_{\text {SN } 2}=347,5 \mathrm{~kJ} / \mathrm{kg} \\
& h_{\text {SO2 }}=317,5 \mathrm{~kJ} / \mathrm{kg} \\
& h_{\text {Shp }}=2553 \mathrm{~kJ} / \mathrm{kg}
\end{aligned}
$$

Entropia na saída:

$$
\begin{aligned}
& S_{s \mathrm{CO}_{2}}=5,522 \mathrm{~kJ} / \mathrm{kgK} \\
& S_{S H 2 O}=11,78 \mathrm{~kJ} / \mathrm{kgK} \\
& s_{s N^{2}}=7,53 \mathrm{~kJ} / \mathrm{kgK} \\
& s_{s o 2}=7,042 \mathrm{~kJ} / \mathrm{kgK} \\
& s_{s h p}=5,189 \mathrm{~kJ} / \mathrm{kgK}
\end{aligned}
$$

\subsection{Análise exergética}

Exergia dos fluxos de massa na entrada:

$$
\begin{aligned}
& e_{e_{i}}=\left(h_{e_{i}}-h_{0_{i}}\right)-T_{0}\left(\mathrm{Se}_{e_{i}}-\mathrm{SO}_{0_{i}}\right),\left(i=h p, \mathrm{CO}_{2}, \mathrm{H}_{2} \mathrm{O}, \mathrm{N}_{2}, \mathrm{O}_{2}\right) \\
& e_{\text {eсо } 2}=((-8229)-(-8949))-288,7(5,93-4,826)=508 \mathrm{~kJ} / \mathrm{kg} \\
& e_{\text {ен } 2 о}=((-12126)-(-13440))-288,7(12,53-10,41)=899,7 \mathrm{~kJ} / \mathrm{kg} \\
& e_{e 2}=(552,8-(-9,829))-288,7(7,92-6,799)=340,4 \mathrm{~kJ} / \mathrm{kg} \\
& e_{e o 2}=(509,4-(-8,713))-288,7(7,408-6,374)=314,5 \mathrm{~kJ} / \mathrm{kg} \\
& e_{\text {ehp }}=(1464-65,34)-288,7(5,189-0,2323)=1056 \mathrm{~kJ} / \mathrm{kg}
\end{aligned}
$$

Exergia dos fluxos de massa na saída: 
$e_{s i}=\left(h_{s i}-h_{0}\right)-T_{0}\left(s_{s i}-s_{0}\right),\left(i=h p, C_{2}, H_{2} O, N_{2}, O_{2}\right)$

(Eq. 2.14)

$e_{s C O 2}=((-8229)-(-8949))-288,7(5,811-4,826)=436,3 \mathrm{~kJ} / \mathrm{kg}$

$e_{\text {SH } 2 O}=((-12126)-(-13440))-288,7(12,31-10,41)=766,1 \mathrm{~kJ} / \mathrm{kg}$

$e_{s N 2}=(552,8-(-9,829))-288,7(7,81-6,799)=270,7 \mathrm{~kJ} / \mathrm{kg}$

$e_{s o 2}=(509,4-(-8,713))-288,7(7,304-6,374)=249,6 \mathrm{~kJ} / \mathrm{kg}$

$e_{\text {shp }}=(3094-65,34)-288,7(3,438-0,2323)=473,3 \mathrm{~kJ} / \mathrm{kg}$

Matéria prima:

$E_{m}=\sum \dot{m}_{i} e_{e i}\left(i=h p, \mathrm{CO}_{2}, \mathrm{H}_{2} \mathrm{O}, \mathrm{N}_{2}, \mathrm{O}_{2}\right)$

$E_{m}=33,59 \cdot 436,3+27,51 \cdot 766,1+329,6 \cdot 270,7+51,29 \cdot 249,6+88,24 \cdot 473,3=179505 \mathrm{~kW}$

Produtos:

$E_{p}=\dot{m}_{s h n} e_{s h p}$

(Eq. 3.76)

$E_{p}=88,24 \cdot 10,56=93216 \mathrm{~kW}$

Perdas:

$E_{l}=\sum \dot{m}_{i} e_{s i},\left(i=\mathrm{CO}_{2}, \mathrm{H}_{2} \mathrm{O}, \mathrm{N}_{2}, \mathrm{O}_{2}\right)$

(Eq. 3.77)

$E_{l}=33,59 \cdot 309,7+27,51 \cdot 530,5+329,6 \cdot 146,2+51,29 \cdot 133,3=80010 \mathrm{~kW}$

Exergia dissipada:

$\dot{E}_{d}=\dot{E}_{m}-\left(\dot{E}_{p}+\dot{E}_{l}\right)$

$\dot{E}_{d}=179505-(93216+80010)=6279 \mathrm{~kW}$

Eficiência exergética:

$$
\begin{aligned}
& \varepsilon=\frac{\dot{E}_{p}}{\dot{E}_{m}} \\
& \varepsilon=\frac{93216}{179505}=0,52
\end{aligned}
$$

Eficiência exergética maior:

$$
\begin{aligned}
\varepsilon^{+} & =\frac{\dot{E}_{p}+\dot{E}_{l}}{\dot{E}_{m}} \\
\varepsilon^{+} & =\frac{93216+80010}{179505}=0,96
\end{aligned}
$$

4.4.1.8 Volume de controle entre os pontos 7 e 8 (Superaquecedor LP $-2^{\circ}$ passo e Superaquecedor $\mathrm{MP}-2^{\circ}$ passo)

\subsection{Condições na entrada}

Temperatura na entrada:

$T_{e_{g}}=361,3^{\circ} \mathrm{C}$

(Gases) 
$T_{e l p}=265,6^{\circ} \mathrm{C}$

(Vapor)

$T_{e m p}=265,8^{\circ} \mathrm{C}$

(Vapor)

Pressão na entrada:

$$
\begin{aligned}
& p_{e^{\prime p}}=480,6 \mathrm{kPa} \\
& p_{e_{m p}}=3521 \mathrm{kPa} \\
& p_{e_{g}}=\frac{103,3}{(1-0,03)^{4} \cdot(1-0,03)^{2} \cdot(1-0,03)^{4}}=140,2 \mathrm{kPa}
\end{aligned}
$$

Entalpia na entrada:

Valores das entalpias obtidos pelo programa EES já reduzidos pelo coeficiente igual a 0,02.

$$
\begin{aligned}
& h_{\text {eсо }}=-8439 \mathrm{~kJ} / \mathrm{kg} \\
& h_{\text {eн } 20}=-12515 \mathrm{~kJ} / \mathrm{kg} \\
& h_{\text {eN } 2}=347,5 \mathrm{~kJ} / \mathrm{kg} \\
& h_{\text {eо } 2}=317,5 \mathrm{~kJ} / \mathrm{kg} \\
& h_{\text {elp }}=2993 \mathrm{~kJ} / \mathrm{kg} \\
& h_{\text {emp }}=2878 \mathrm{~kJ} / \mathrm{kg}
\end{aligned}
$$

Entropia na entrada:

$$
\begin{aligned}
& \mathrm{SeCO}_{2}=5,522 \mathrm{~kJ} / \mathrm{kgK} \\
& \text { Sен } 2 \text { о }=11,78 \mathrm{~kJ} / \mathrm{kgK} \\
& S_{e_{N} 2}=7,53 \mathrm{~kJ} / \mathrm{kgK} \\
& S_{e o 2}=7,042 \mathrm{~kJ} / \mathrm{kgK} \\
& S_{e l p}=7,35 \mathrm{~kJ} / \mathrm{kgK} \\
& S_{e_{m p}}=6,625 \mathrm{~kJ} / \mathrm{kgK}
\end{aligned}
$$

\subsection{Equações de balanço}

\section{Balanço de Massa}

Valor da vazão mássica associada a cada substância presente no volume de controle considerado.

$$
\begin{aligned}
& \dot{m}_{e C O 2}=33,59 \mathrm{~kg} / \mathrm{s} \\
& \dot{m}_{e H 2 O}=27,51 \mathrm{~kg} / \mathrm{s} \\
& \dot{m}_{e N 2}=329,6 \mathrm{~kg} / \mathrm{s} \\
& \dot{m}_{e O 2}=51,29 \mathrm{~kg} / \mathrm{s} \\
& \dot{m}_{e l p}=3,604 \mathrm{~kg} / \mathrm{s} \\
& \dot{m}_{e^{m p}}=4,35 \mathrm{~kg} / \mathrm{s} \\
& \dot{m}_{s i}=\dot{m}_{e i},\left(\mathrm{i}=\mathrm{hp} ; \mathrm{mp} ; \mathrm{CO}_{2} ; \mathrm{H}_{2} \mathrm{O} ; \mathrm{N}_{2} ; \mathrm{O}_{2}\right)
\end{aligned}
$$

Balanço de energia

Da mesma forma como no balanço de massa, não será necessário determinar nenhuma variável pelo balanço de energia para este volume de controle.

\subsection{Condições na saída}


Temperatura na saída:

$T_{s_{g}}=358,2^{\circ} \mathrm{C}$

(Gases)

$T_{s i p}=305,9{ }^{\circ} \mathrm{C}$

$T_{\text {smp }}=355,3{ }^{\circ} \mathrm{C}$

(Vapor)

Pressão na saída:

$p_{s p}=459,2 \mathrm{kPa}$

$p_{\text {smp }}=3511 \mathrm{kPa}$

$p_{s_{g}}=\frac{103,3}{(1-0,03)^{4} \cdot(1-0,03)^{2} \cdot(1-0,03)^{2}}=131,9 \mathrm{kPa}$

Entalpia na saída:

$$
\begin{aligned}
& h_{S \mathrm{CO}_{2}}=-8442 \mathrm{~kJ} / \mathrm{kg} \\
& h_{S H 2 O}=-12521 \mathrm{~kJ} / \mathrm{kg} \\
& h_{S N^{2}}=344,2 \mathrm{~kJ} / \mathrm{kg} \\
& h_{s O 2}=314,4 \mathrm{~kJ} / \mathrm{kg} \\
& h_{S l p}=3077 \mathrm{~kJ} / \mathrm{kg} \\
& h_{\text {smp }}=3116 \mathrm{~kJ} / \mathrm{kg}
\end{aligned}
$$

Entropia na saída:

$$
\begin{aligned}
& S_{s \mathrm{CO}_{2}}=5,528 \mathrm{~kJ} / \mathrm{kgK} \\
& S_{S H 2 O}=11,79 \mathrm{~kJ} / \mathrm{kgK} \\
& s_{s 2}=7,543 \mathrm{~kJ} / \mathrm{kgK} \\
& S_{s o 2}=7,053 \mathrm{~kJ} / \mathrm{kgK} \\
& s_{s l p}=7,521 \mathrm{~kJ} / \mathrm{kgK} \\
& s_{\text {smp }}=6,676 \mathrm{~kJ} / \mathrm{kgK}
\end{aligned}
$$

\subsection{Análise exergética}

Exergia dos fluxos de massa na entrada:

$$
\begin{aligned}
& e_{e_{i}}=\left(h_{e_{i}}-h_{0_{i}}\right)-T_{0}\left(s_{e_{i}}-S 0_{i}\right),\left(\mathrm{i}=\mathrm{hp}, \mathrm{mp}, \mathrm{CO}_{2}, \mathrm{H}_{2} \mathrm{O}, \mathrm{N}_{2}, \mathrm{O}_{2}\right) \\
& e_{\text {eсо }}=((-8439)-(-8949))-288,7(5,522-4,826)=309,7 \mathrm{~kJ} / \mathrm{kg} \\
& e_{\text {ен } 20}=((-12515)-(-13440))-288,7(11,78-10,41)=530,5 \mathrm{~kJ} / \mathrm{kg} \\
& e_{\text {eN2 }}=(347,5-(-9,829))-288,7(7,53-6,799)=146,2 \mathrm{~kJ} / \mathrm{kg} \\
& e_{\text {eо口 }}=(317,5-(-8,713))-288,7(7,042-6,374)=133,3 \mathrm{~kJ} / \mathrm{kg} \\
& e_{e_{l p}}=(2993-65,34)-288,7(7,35-0,2323)=872,9 \mathrm{~kJ} / \mathrm{kg} \\
& e_{\text {emp }}=(2878-65,34)-288,7(6,265-0,2323)=1071 \mathrm{~kJ} / \mathrm{kg}
\end{aligned}
$$

Exergia dos fluxos de massa na saída:

$$
\begin{aligned}
& e_{s i}=\left(h_{s i}-h_{0 i}\right)-T_{0}\left(s_{s i}-s 0_{i}\right),\left(\mathrm{i}=\mathrm{hp}, \mathrm{mp}, \mathrm{CO}_{2}, \mathrm{H}_{2} \mathrm{O}, \mathrm{N}_{2}, \mathrm{O}_{2}\right) \\
& e_{\text {sCO2 }}=((-8442)-(-8949))-288,7(5,528-4,826)=304,6 \mathrm{~kJ} / \mathrm{kg} \\
& e_{\text {SH } 2 O}=((-12521)-(-13440))-288,7(11,79-10,41)=519,1 \mathrm{~kJ} / \mathrm{kg} \\
& e_{N 2}=(344,2-(-9,829))-288,7(7,543-6,799)=139,2 \mathrm{~kJ} / \mathrm{kg} \\
& e_{s o 2}=(314,4-(-8,713))-288,7(7,053-6,374)=127,1 \mathrm{~kJ} / \mathrm{kg} \\
& e_{s l_{p}}=(3077-65,34)-288,7(7,521-0,2323)=907,4 \mathrm{~kJ} / \mathrm{kg} \\
& e_{\text {smp }}=(3116-65,34)-288,7(6,676-0,2323)=1190 \mathrm{~kJ} / \mathrm{kg}
\end{aligned}
$$


Matéria prima:

$E_{m}=\sum \dot{m}_{i} e_{e i}\left(\mathrm{i}=\mathrm{hp}, \mathrm{mp}, \mathrm{CO}_{2}, \mathrm{H}_{2} \mathrm{O}, \mathrm{N}_{2}, \mathrm{O}_{2}\right)$

$E_{m}=33,59 \cdot 309.7+27,51 \cdot 530,5+329,6 \cdot 146,2+51,29 \cdot 133,3+3,604 \cdot 872,9+4,35 \cdot 1071=87814 \mathrm{~kW}$

Produtos:

$E_{p}=\dot{m}_{s l p} e_{s l p}+\dot{m}_{s m p} e_{s m p}$

$E_{p}=3,604 \cdot 907,4+4,35 \cdot 1190=8448 \mathrm{~kW}$

Perdas:

$E_{l}=\sum \dot{m}_{i} e_{s i},\left(i=\mathrm{CO}_{2}, \mathrm{H}_{2} \mathrm{O}, \mathrm{N}_{2}, \mathrm{O}_{2}\right)$

$E_{l}=33,59 \cdot 304,6+27,51 \cdot 519,1+329,6 \cdot 139,2+51,29 \cdot 127,1=76901 \mathrm{~kW}$

Exergia dissipada:

$\dot{E}_{d}=\dot{E}_{m}-\left(\dot{E}_{p}+\dot{E}_{l}\right)$

$\dot{E}_{d}=87814-(8448+76901)=2465 \mathrm{~kW}$

Eficiência exergética:

$$
\begin{aligned}
& \varepsilon=\frac{\dot{E}_{p}}{\dot{E}_{m}} \\
& \varepsilon=\frac{8448}{87814}=0,10
\end{aligned}
$$

Eficiência exergética maior:

$$
\begin{aligned}
& \varepsilon^{+}=\frac{\dot{E}_{p}+\dot{E}_{l}}{\dot{E}_{m}} \\
& \varepsilon^{+}=\frac{8448+76901}{87814}=0,97
\end{aligned}
$$

4.4.1.9 Volume de controle entre os pontos 8 e 9 (Economizador $\mathrm{HP}-2^{\circ}$ passo)

\subsection{Condições na entrada}

Temperatura na entrada:

$T_{e_{g}}=358,2^{\circ} \mathrm{C}$

$T_{\text {ehp }}=222,7^{\circ} \mathrm{C}$

(Vapor)

Pressão na entrada:

$p_{\text {ehp }}=17253 \mathrm{kPa}$

$p_{e_{g}}=\frac{103,3}{(1-0,03)^{4} \cdot(1-0,03)^{2} \cdot(1-0,03)^{2}}=131,9 \mathrm{kPa}$

Entalpia na entrada: 


$$
\begin{aligned}
& h_{\text {eсо } 2}=-8615 \mathrm{~kJ} / \mathrm{kg} \\
& h_{\text {eн } 20}=-12777 \mathrm{~kJ} / \mathrm{kg} \\
& h_{\text {eN2 }}=351,2 \mathrm{~kJ} / \mathrm{kg} \\
& h_{\text {eо } 2}=320,8 \mathrm{~kJ} / \mathrm{kg} \\
& h_{\text {ehp }}=960,2 \mathrm{~kJ} / \mathrm{kg}
\end{aligned}
$$

Entropia na entrada:

$$
\begin{aligned}
& \text { Sесо } 2=5,528 \mathrm{~kJ} / \mathrm{kgK} \\
& S_{\text {eн } 20}=11,79 \mathrm{~kJ} / \mathrm{kgK} \\
& S_{\text {N } 2^{2}}=7,543 \mathrm{~kJ} / \mathrm{kgK} \\
& S_{\text {eо } 2}=7,053 \mathrm{~kJ} / \mathrm{kgK} \\
& S_{\text {ehp }}=2,515 \mathrm{~kJ} / \mathrm{kgK}
\end{aligned}
$$

\subsection{Equações de balanço}

\section{Balanço de Massa}

Valor da vazão mássica associada a cada substância presente no volume de controle considerado.

$$
\begin{aligned}
& \dot{m}_{\text {eСO2 }}=33,59 \mathrm{~kg} / \mathrm{s} \\
& \dot{m}_{\text {eH } 2 O}=27,51 \mathrm{~kg} / \mathrm{s} \\
& \dot{m}_{e N 2}=329,6 \mathrm{~kg} / \mathrm{s} \\
& \dot{m}_{e O 2}=51,29 \mathrm{~kg} / \mathrm{s} \\
& \dot{m}_{e h p}=88,24 \mathrm{~kg} / \mathrm{s} \\
& \dot{m}_{s i}=\dot{m}_{e i},\left(\mathrm{i}=\mathrm{hp} ; \mathrm{CO}_{2} ; \mathrm{H}_{2} \mathrm{O} ; \mathrm{N}_{2} ; \mathrm{O}_{2}\right)
\end{aligned}
$$

Balanço de energia

Da mesma forma como no balanço de massa, não será necessário determinar nenhuma variável pelo balanço de energia para este volume de controle.

\subsection{Condições na saída}

Temperatura na saída:

$$
T_{s g}=267,9^{\circ} \mathrm{C}
$$

$T_{s h p}=322,4^{\circ} \mathrm{C}$

Pressão na saída:

$$
\begin{aligned}
& p_{s l p}=17073 \mathrm{kPa} \\
& p_{s_{g}}=\frac{103,3}{(1-0,03)^{3} \cdot(1-0,03)^{2} \cdot(1-0,03)^{2}}=131,9 \mathrm{kPa}
\end{aligned}
$$

Entalpia na saída:

$$
\begin{aligned}
& h_{s \mathrm{CO}_{2}}=-8711 \mathrm{~kJ} / \mathrm{kg} \\
& h_{S \mathrm{H} 2 \mathrm{O}}=-12958 \mathrm{~kJ} / \mathrm{kg} \\
& h_{S \mathrm{~N}_{2}}=254,3 \mathrm{~kJ} / \mathrm{kg} \\
& h_{\mathrm{SO}_{2}}=230,4 \mathrm{~kJ} / \mathrm{kg}
\end{aligned}
$$


$h_{s h p}=1464 \mathrm{~kJ} / \mathrm{kg}$

(EES)

Entropia na saída:

$$
\begin{aligned}
& S_{s \mathrm{sO}_{2}}=5,369 \mathrm{~kJ} / \mathrm{kgK} \\
& S_{S \mathrm{H} 2 \mathrm{O}}=11,5 \mathrm{~kJ} / \mathrm{kgK} \\
& s_{S N 2}=7,386 \mathrm{~kJ} / \mathrm{kgK} \\
& s_{s o 2}=6,907 \mathrm{~kJ} / \mathrm{kgK} \\
& s_{s h p}=3,438 \mathrm{~kJ} / \mathrm{kgK}
\end{aligned}
$$

\subsection{Análise exergética}

Exergia dos fluxos de massa na entrada:

$$
\begin{aligned}
& e_{e_{i}}=\left(h_{e_{i}}-h_{0_{i}}\right)-T_{0}\left(S_{e_{i}}-S_{0 i}\right),\left(i=h p, \mathrm{CO}_{2}, \mathrm{H}_{2} \mathrm{O}, \mathrm{N}_{2}, \mathrm{O}_{2}\right) \\
& e_{\text {есо } 2}=((-8615)-(-8949))-288,7(5,528-4,826)=132,3 \mathrm{~kJ} / \mathrm{kg} \\
& e_{\text {ен } 20}=((-12777)-(-13440))-288,7(11,79-10,41)=263,6 \mathrm{~kJ} / \mathrm{kg} \\
& e_{\text {eN } 2}=(351,2-(-9,829))-288,7(7,543-6,799)=146,2 \mathrm{~kJ} / \mathrm{kg} \\
& e_{e о 2}=(320,8-(-8,713))-288,7(7,053-6,374)=133,5 \mathrm{~kJ} / \mathrm{kg} \\
& e_{\text {ehp }}=(960,2-65,34)-288,7(2,515-0,2323)=235,7 \mathrm{~kJ} / \mathrm{kg}
\end{aligned}
$$

Exergia dos fluxos de massa na saída:

$$
\begin{aligned}
& e_{s i}=\left(h_{s i}-h_{0 i}\right)-T_{0}\left(s_{s i}-s_{0 i}\right),\left(i=h p, \mathrm{CO}_{2}, \mathrm{H}_{2} \mathrm{O}, \mathrm{N}_{2}, \mathrm{O}_{2}\right) \\
& e_{s \mathrm{SO}_{2}}=((-8711)-(-8949))-288,7(5,369-4,826)=81,86 \mathrm{~kJ} / \mathrm{kg} \\
& e_{S H 2 O}=((-12958)-(-13440))-288,7(11,5-10,41)=167,8 \mathrm{~kJ} / \mathrm{kg} \\
& e_{S N_{2}}=(254,3-(-9,829))-288,7(7,386-6,799)=94,47 \mathrm{~kJ} / \mathrm{kg} \\
& e_{s O 2}=(230,4-(-8,713))-288,7(6,907-6,374)=85,45 \mathrm{~kJ} / \mathrm{kg} \\
& e_{s h p}=(1464-65,34)-288,7(3,438-0,2323)=473,3 \mathrm{~kJ} / \mathrm{kg}
\end{aligned}
$$

Matéria prima:

$E_{m}=\sum \dot{m}_{i} e_{e i}\left(i=h p, \mathrm{CO}_{2}, \mathrm{H}_{2} \mathrm{O}, \mathrm{N}_{2}, \mathrm{O}_{2}\right)$

$E_{m}=33,59 \cdot 132,3+27,51 \cdot 263,6+329,6 \cdot 146,2+51,29 \cdot 133,5+88,24 \cdot 235,7=87523 \mathrm{~kW}$

Produtos:

$$
\begin{aligned}
& E_{p}=\dot{m}_{s h e_{s h p}} \\
& E_{p}=88,24 \cdot 473,3=41761 \mathrm{~kW}
\end{aligned}
$$

Perdas:

$$
\begin{aligned}
& E_{l}=\sum \dot{m}_{i} e_{s i},\left(i=\mathrm{CO}_{2}, \mathrm{H}_{2} \mathrm{O}, \mathrm{N}_{2}, \mathrm{O}_{2}\right) \\
& E_{l}=33,59 \cdot 81,86+27,51 \cdot 167,8+329,6 \cdot 94,47+51,29 \cdot 85,45=42885 \mathrm{~kW}
\end{aligned}
$$

Exergia dissipada:

$$
\begin{aligned}
& \dot{E}_{d}=\dot{E}_{m}-\left(\dot{E}_{p}+\dot{E}_{l}\right) \\
& \dot{E}_{d}=87523-(41761+42885)=2877 \mathrm{~kW}
\end{aligned}
$$


Eficiência exergética:

$$
\begin{aligned}
& \varepsilon=\frac{\dot{E}_{p}}{\dot{E}_{m}} \\
& \varepsilon=\frac{41761}{87523}=0,48
\end{aligned}
$$

Eficiência exergética maior:

$$
\begin{aligned}
\varepsilon^{+} & =\frac{\dot{E}_{p}+\dot{E}_{l}}{\dot{E}_{m}} \\
\varepsilon^{+} & =\frac{41761+42885}{87523}=0,97
\end{aligned}
$$

4.4.1.10 Volume de controle entre os pontos 9 e 10 (Superaquecedor MP $-1^{\circ}$ passo e Superaquecedor $\mathrm{LP}-1^{\circ}$ passo)

\subsection{Condições na entrada}

Temperatura na entrada:

$T_{e_{g}}=267,9^{\circ} \mathrm{C}$

$T_{e l p}=152^{\circ} \mathrm{C}$

$T_{\text {emp }}=237,6^{\circ} \mathrm{C}$

Pressão na entrada:

$$
\begin{aligned}
& p_{e_{l p}}=501,9 \mathrm{kPa} \\
& p_{e_{m p}}=3531 \mathrm{kPa} \\
& p_{e g}=\frac{103,3}{(1-0,03)^{3} \cdot(1-0,03)^{2} \cdot(1-0,03)^{2}}=127,9 \mathrm{kPa}
\end{aligned}
$$

Entalpia na entrada:

$$
\begin{aligned}
& h_{\text {есо }}=-8711 \mathrm{~kJ} / \mathrm{kg} \\
& h_{\text {ен } 2 о}=-12958 \mathrm{~kJ} / \mathrm{kg} \\
& h_{\text {eN } 2}=254,3 \mathrm{~kJ} / \mathrm{kg} \\
& h_{\text {eо口 }}=230,4 \mathrm{~kJ} / \mathrm{kg} \\
& h_{\text {elp }}=2749 \mathrm{~kJ} / \mathrm{kg} \\
& h_{\text {emp }}=2803 \mathrm{~kJ} / \mathrm{kg}
\end{aligned}
$$

Entropia na entrada:

$$
\begin{aligned}
& S_{\text {eco }}=5,369 \mathrm{~kJ} / \mathrm{kgK} \\
& \text { Sен } 2 \text { о }=11,5 \mathrm{~kJ} / \mathrm{kgK} \\
& S_{e N 2}=7,386 \mathrm{~kJ} / \mathrm{kgK} \\
& \mathrm{SeO} 2=6,907 \mathrm{~kJ} / \mathrm{kgK} \\
& S_{e l p}=6,82 \mathrm{~kJ} / \mathrm{kgK} \\
& S_{e_{m p}}=6,16 \mathrm{~kJ} / \mathrm{kgK}
\end{aligned}
$$

\subsection{Equações de balanço}

Balanço de Massa 
Valor da vazão mássica associada a cada substância presente no volume de controle considerado.

$$
\begin{aligned}
& \dot{m}_{e C O 2}=33,59 \mathrm{~kg} / \mathrm{s} \\
& \dot{m}_{e H 2 O}=27,51 \mathrm{~kg} / \mathrm{s} \\
& \dot{m}_{e N 2}=329,6 \mathrm{~kg} / \mathrm{s} \\
& \dot{m}_{e O 2}=51,29 \mathrm{~kg} / \mathrm{s} \\
& \dot{m}_{e l p}=3,604 \mathrm{~kg} / \mathrm{s} \\
& \dot{m}_{e^{m p}}=4,35 \mathrm{~kg} / \mathrm{s} \\
& \dot{m}_{s i}=\dot{m}_{e i},\left(\mathrm{i}=\mathrm{hp} ; \mathrm{mp} ; \mathrm{CO}_{2} ; \mathrm{H}_{2} \mathrm{O} ; \mathrm{N}_{2} ; \mathrm{O}_{2}\right)
\end{aligned}
$$

Balanço de energia

Da mesma forma como no balanço de massa, não será necessário determinar nenhuma variável pelo balanço de energia para este volume de controle.

\subsection{Condições na saída}

Temperatura na saída:

$T_{s g}=265,8^{\circ} \mathrm{C}$

(Gases)

$T_{s l p}=265,6^{\circ} \mathrm{C}$

$T_{\text {smp }}=265,8^{\circ} \mathrm{C}$

(Vapor)

Pressão na saída:

$$
\begin{aligned}
& p_{s l p}=480,6 \mathrm{kPa} \\
& p_{s m p}=3521 \mathrm{kPa} \\
& p_{s g}=\frac{103,3}{(1-0,03)^{3} \cdot(1-0,03)^{2} \cdot(1-0,03)^{0}}=120,4 \mathrm{kPa}
\end{aligned}
$$

Entalpia na saída:

$$
\begin{aligned}
& h_{\text {sCO2 }}=-8713 \mathrm{~kJ} / \mathrm{kg} \\
& h_{\text {sH } 2 O}=-12962 \mathrm{~kJ} / \mathrm{kg} \\
& h_{s N 2}=252,1 \mathrm{~kJ} / \mathrm{kg} \\
& h_{\text {sO2 }}=228,3 \mathrm{~kJ} / \mathrm{kg} \\
& h_{s l p}=2993 \mathrm{~kJ} / \mathrm{kg} \\
& h_{\text {smp }}=2878 \mathrm{~kJ} / \mathrm{kg}
\end{aligned}
$$

Entropia na saída:

$$
\begin{aligned}
& S_{s \mathrm{CO}_{2}}=5,376 \mathrm{~kJ} / \mathrm{kgK} \\
& S_{S H_{2 O} O}=11,52 \mathrm{~kJ} / \mathrm{kgK} \\
& s_{s N_{2}}=7,4 \mathrm{~kJ} / \mathrm{kgK} \\
& s_{s o 2}=6,919 \mathrm{~kJ} / \mathrm{kgK} \\
& s_{s \mid p}=7,35 \mathrm{~kJ} / \mathrm{kgK} \\
& s_{s m p}=6,265 \mathrm{~kJ} / \mathrm{kgK}
\end{aligned}
$$


Exergia dos fluxos de massa na entrada:

$$
\begin{aligned}
& e_{e_{i}}=\left(h_{e_{i}}-h_{0_{i}}\right)-T_{0}\left(S_{e_{i}}-S_{0 i}\right),\left(\mathrm{i}=\mathrm{hp}, \mathrm{mp}, \mathrm{CO}_{2}, \mathrm{H}_{2} \mathrm{O}, \mathrm{N}_{2}, \mathrm{O}_{2}\right) \\
& e_{\text {eсо2 }}=((-8711)-(-8949))-288,7(5,369-4,826)=81,86 \mathrm{~kJ} / \mathrm{kg} \\
& e_{\text {eн } 2 о}=((-12958)-(-13440))-288,7(11,5-10,41)=167,8 \mathrm{~kJ} / \mathrm{kg} \\
& e_{\text {eN2 }}=(254,3-(-9,829))-288,7(7,386-6,799)=94,47 \mathrm{~kJ} / \mathrm{kg} \\
& e_{e о 2}=(230,4-(-8,713))-288,7(6,907-6,374)=85,45 \mathrm{~kJ} / \mathrm{kg} \\
& e_{e l p}=(2749-65,34)-288,7(6,82-0,2323)=785,1 \mathrm{~kJ} / \mathrm{kg} \\
& e_{\text {emp }}=(2803-65,34)-288,7(6,16-0,2323)=1027 \mathrm{~kJ} / \mathrm{kg}
\end{aligned}
$$

Exergia dos fluxos de massa na saída:

$$
\begin{aligned}
& e_{s i}=\left(h_{s i}-h_{0 i}\right)-T_{0}\left(s_{s i}-s 0_{i}\right),\left(\mathrm{i}=\mathrm{hp}, \mathrm{mp}, \mathrm{CO}_{2}, \mathrm{H}_{2} \mathrm{O}, \mathrm{N}_{2}, \mathrm{O}_{2}\right) \\
& e_{\text {sCO2 }}=((-8713)-(-8949))-288,7(5,376-4,826)=77,52 \mathrm{~kJ} / \mathrm{kg} \\
& e_{\text {SH } 2 O}=((-12962)-(-13440))-288,7(11,52-10,41)=157,7 \mathrm{~kJ} / \mathrm{kg} \\
& e_{N_{2} 2}=(252,1-(-9,829))-288,7(7,4-6,799)=88,21 \mathrm{~kJ} / \mathrm{kg} \\
& e_{s o 2}=(228,3-(-8,713))-288,7(6,919-6,374)=79,91 \mathrm{~kJ} / \mathrm{kg} \\
& e_{s l p}=(2993-65,34)-288,7(7,35-0,2323)=872,9 \mathrm{~kJ} / \mathrm{kg} \\
& e_{\text {smp }}=(2878-65,34)-288,7(6,265-0,2323)=1071 \mathrm{~kJ} / \mathrm{kg}
\end{aligned}
$$

Matéria prima:

$$
\begin{aligned}
& E_{m}=\sum \dot{m}_{i} e_{e i}\left(\mathrm{i}=1 \mathrm{p}, \mathrm{mp}, \mathrm{CO}_{2}, \mathrm{H}_{2} \mathrm{O}, \mathrm{N}_{2}, \mathrm{O}_{2}\right) \\
& E_{m}=33,59 \cdot 81,86+27,51 \cdot 167,8+329,6 \cdot 94,47+51,29 \cdot 85,45+3,604 \cdot 781,5+4,35 \cdot 1027=50166 \mathrm{~kW}
\end{aligned}
$$

Produtos:

$$
\begin{aligned}
& E_{p}=\dot{m}_{s l p} e_{s l p}+\dot{m}_{s m p} e_{s m p} \\
& E_{p}=3,604 \cdot 872,9+4,35 \cdot 1071=7805 \mathrm{~kW}
\end{aligned}
$$

Perdas:

$$
\begin{aligned}
& E_{l}=\sum \dot{m}_{i} e_{s i},\left(i=\mathrm{CO}_{2}, \mathrm{H}_{2} \mathrm{O}, N_{2}, \mathrm{O}_{2}\right) \\
& E_{l}=33,59 \cdot 77,52+27,51 \cdot 157,7+329,6 \cdot 88,21+51,29 \cdot 79,91=40113 \mathrm{~kW}
\end{aligned}
$$

Exergia dissipada:

$$
\begin{aligned}
& \dot{E}_{d}=\dot{E}_{m}-\left(\dot{E}_{p}+\dot{E}_{l}\right) \\
& \dot{E}_{d}=50166-(7805+40113)=2248 \mathrm{~kW}
\end{aligned}
$$

Eficiência exergética:

$$
\begin{aligned}
& \varepsilon=\frac{\dot{E}_{p}}{\dot{E}_{m}} \\
& \varepsilon=\frac{7805}{50166}=0,15
\end{aligned}
$$


Eficiência exergética maior:

$$
\begin{aligned}
& \varepsilon^{+}=\frac{\dot{E}_{p}+\dot{E}_{l}}{\dot{E}_{m}} \\
& \varepsilon^{+}=\frac{7805+40113}{50166}=0,95
\end{aligned}
$$

4.4.1.11 Volume de controle entre os pontos 10 e 11 (Gerador de vapor MP)

4.4.1.11.1 Condições na entrada

Temperatura na entrada:

$T_{e_{g}}=265,8^{\circ} \mathrm{C}$

$T_{\text {emp }}=237,6^{\circ} \mathrm{C}$

Pressão na entrada:

$$
\begin{aligned}
& p_{e_{m p}}=3541 \mathrm{kPa} \\
& p_{e_{g}}=\frac{103,3}{(1-0,03)^{3} \cdot(1-0,03)^{2} \cdot(1-0,03)^{0}}=120,4 \mathrm{kPa}
\end{aligned}
$$

Entalpia na entrada:

$$
\begin{aligned}
& h_{\text {есо } 2}=-8713 \mathrm{~kJ} / \mathrm{kg} \\
& h_{\text {ен } 20}=-12962 \mathrm{~kJ} / \mathrm{kg} \\
& h_{\text {eN2 } 2}=252,1 \mathrm{~kJ} / \mathrm{kg} \\
& h_{\text {eо } 2}=228,3 \mathrm{~kJ} / \mathrm{kg} \\
& h_{\text {emp }}=1026 \mathrm{~kJ} / \mathrm{kg}
\end{aligned}
$$

Entropia na entrada:

$$
\begin{aligned}
& \text { Sесо }_{2}=5,376 \mathrm{~kJ} / \mathrm{kgK} \\
& \text { Sен } 2 о=11,52 \mathrm{~kJ} / \mathrm{kgK} \\
& S_{\text {N }_{2}}=7,4 \mathrm{~kJ} / \mathrm{kgK} \\
& S_{\text {eо } 2}=6,919 \mathrm{~kJ} / \mathrm{kgK} \\
& S_{\text {emp }}=2,678 \mathrm{~kJ} / \mathrm{kgK}
\end{aligned}
$$

\subsection{Equações de balanço}

Balanço de Massa considerado.

Valor da vazão mássica associada a cada substância presente no volume de controle

$$
\begin{aligned}
& \dot{m}_{\text {eсо2 }}=33,59 \mathrm{~kg} / \mathrm{s} \\
& \dot{m}_{e \mathrm{H} 2 O}=27,51 \mathrm{~kg} / \mathrm{s} \\
& \dot{m}_{e N 2}=329,6 \mathrm{~kg} / \mathrm{s} \\
& \dot{m}_{e O 2}=51,29 \mathrm{~kg} / \mathrm{s} \\
& \dot{m}_{e m p}=4,349 \mathrm{~kg} / \mathrm{s} \\
& \dot{m}_{s i}=\dot{m}_{e i},\left(\mathrm{i}=\mathrm{mp}, \mathrm{CO}_{2} ; \mathrm{H}_{2} \mathrm{O} ; \mathrm{N}_{2} ; \mathrm{O}_{2}\right)
\end{aligned}
$$




\section{Balanço de energia}

Da mesma forma como no balanço de massa, não será necessário determinar nenhuma variável pelo balanço de energia para este volume de controle.

\subsection{Condições na saída}

Temperatura na saída:

$T_{s_{g}}=250^{\circ} \mathrm{C}$

(Gases)

$T_{\text {smp }}=237,6^{\circ} \mathrm{C}$

(Vapor)

Pressão na saída:

$p_{s m p}=3531 \mathrm{kPa}$

$p_{s g}=\frac{103,3}{(1-0,03)^{3} \cdot(1-0,03)^{1} \cdot(1-0,03)^{0}}=116,7 \mathrm{kPa}$

Entalpia na saída:

$$
\begin{aligned}
& h_{\text {SСO } 2}=-8729 \mathrm{~kJ} / \mathrm{kg} \\
& h_{\text {SH } 2 \mathrm{O}}=-12993 \mathrm{~kJ} / \mathrm{kg} \\
& h_{\text {SN } 2}=235,3 \mathrm{~kJ} / \mathrm{kg} \\
& h_{\text {sO } 2}=212,8 \mathrm{~kJ} / \mathrm{kg} \\
& h_{\text {Sm }}=2803 \mathrm{~kJ} / \mathrm{kg}
\end{aligned}
$$

Entropia na saída:

$$
\begin{aligned}
& S_{s \mathrm{CO}_{2}}=5,351 \mathrm{~kJ} / \mathrm{kgK} \\
& S_{\mathrm{SH}_{2 O} \mathrm{O}}=11,47 \mathrm{~kJ} / \mathrm{kgK} \\
& s_{S_{N 2}}=7,378 \mathrm{~kJ} / \mathrm{kgK} \\
& S_{s o 2}=6,897 \mathrm{~kJ} / \mathrm{kgK} \\
& S_{\text {smp }}=6,16 \mathrm{~kJ} / \mathrm{kgK}
\end{aligned}
$$

\subsection{Análise exergética}

Exergia dos fluxos de massa na entrada:

$$
\begin{aligned}
& e_{e_{i}}=\left(h_{e_{i}}-h_{0_{i}}\right)-T_{0}\left(S_{e_{i}}-S_{0}\right),\left(i=m p, \mathrm{CO}_{2}, \mathrm{H}_{2} \mathrm{O}, \mathrm{N}_{2}, \mathrm{O}_{2}\right) \\
& e_{\text {есо2 }}=((-8713)-(-8949))-288,7(5,376-4,826)=77,52 \mathrm{~kJ} / \mathrm{kg} \\
& e_{\text {ен } 2 \mathrm{O}}=((-12962)-(-13440))-288,7(11,52-10,41)=157,7 \mathrm{~kJ} / \mathrm{kg} \\
& e_{\text {eN2 }}=(252,1-(-9,829))-288,7(7,4-6,799)=88,21 \mathrm{~kJ} / \mathrm{kg} \\
& e_{e о 2}=(228,3-(-8,713))-288,7(6,919-6,374)=79,91 \mathrm{~kJ} / \mathrm{kg} \\
& e_{\text {emp }}=(1026-65,34)-288,7(2,678-0,2323)=254,2 \mathrm{~kJ} / \mathrm{kg}
\end{aligned}
$$

Exergia dos fluxos de massa na saída:

$$
\begin{aligned}
& e_{s i}=\left(h_{s i}-h_{0 i}\right)-T_{0}\left(S_{s i}-s_{i}\right),\left(i=m p, C_{2}, H_{2} O, N_{2}, O_{2}\right) \\
& e_{s C O 2}=((-8729)-(-8949))-288,7(5,351-4,826)=68,38 \mathrm{~kJ} / \mathrm{kg} \\
& e_{s H 2 O}=((-12993)-(-13440))-288,7(11,47-10,41)=139,4 \mathrm{~kJ} / \mathrm{kg} \\
& e_{s{ }^{2} 2}=(235,3-(-9,829))-288,7(7,378-6,799)=77,93 \mathrm{~kJ} / \mathrm{kg} \\
& e_{s O_{2}}=(212,8-(-8,713))-288,7(6,897-6,374)=70,52 \mathrm{~kJ} / \mathrm{kg} \\
& e_{s m p}=(2803-65,34)-288,7(6,16-0,2323)=1027 \mathrm{~kJ} / \mathrm{kg}
\end{aligned}
$$

Matéria prima: 
$E_{m}=\sum \dot{m}_{i} e_{e i}\left(i=m p, \mathrm{CO}_{2}, \mathrm{H}_{2} \mathrm{O}, \mathrm{N}_{2}, \mathrm{O}_{2}\right)$

$E_{m}=33,59 \cdot 77,52+27,51 \cdot 157,7+329,6 \cdot 88,21+51,29 \cdot 79,91+4,349 \cdot 254,2=41219 \mathrm{~kW}$

Produtos:

$$
\begin{aligned}
& E_{p}=\dot{m}_{s m p} e_{S m p} \\
& E_{p}=4,349 \cdot 1027=4465 \mathrm{~kW}
\end{aligned}
$$

Perdas:

$$
\begin{aligned}
& E_{l}=\sum \dot{m}_{i} e_{s i},\left(i=\mathrm{CO}_{2}, \mathrm{H}_{2} \mathrm{O}, \mathrm{N}_{2}, \mathrm{O}_{2}\right) \\
& E_{l}=33,59 \cdot 68,38+27,51 \cdot 139,4+329,6 \cdot 77,93+51,29 \cdot 70,52=35435 \mathrm{~kW}
\end{aligned}
$$

Exergia dissipada:

$$
\begin{aligned}
& \dot{E}_{d}=\dot{E}_{m}-\left(\dot{E}_{p}+\dot{E}_{l}\right) \\
& \dot{E}_{d}=41219-(4465+35435)=1319 \mathrm{~kW}
\end{aligned}
$$

Eficiência exergética:

$$
\begin{aligned}
& \varepsilon=\frac{\dot{E}_{p}}{\dot{E}_{m}} \\
& \varepsilon=\frac{4465}{41219}=0,11
\end{aligned}
$$

Eficiência exergética maior:

$$
\begin{aligned}
& \varepsilon^{+}=\frac{\dot{E}_{p}+\dot{E}_{l}}{\dot{E}_{m}} \\
& \varepsilon^{+}=\frac{4465+35435}{41219}=0,97
\end{aligned}
$$

4.4.1.12 Volume de controle entre os pontos 11 e 12 (Economizador $\mathrm{HP}-1^{\circ}$ passo e Economizador MP - passo único)

\subsection{Condições na entrada}

Temperatura na entrada:

$$
\begin{aligned}
& T_{e_{g}}=250^{\circ} \mathrm{C} \\
& T_{e h p}=154,1^{\circ} \mathrm{C} \\
& T_{e m p}=154,2^{\circ} \mathrm{C}
\end{aligned}
$$

Pressão na entrada:

$$
\begin{aligned}
& p_{e_{h p}}=17432 \mathrm{kPa} \\
& p_{\text {emp }}=3551 \mathrm{kPa} \\
& p_{e g}=\frac{103,3}{(1-0,03)^{3} \cdot(1-0,03)^{1} \cdot(1-0,03)^{0}}=116,7 \mathrm{kPa}
\end{aligned}
$$

Entalpia na entrada: 


$$
\begin{aligned}
& h_{\text {есо } 2}=-8729 \mathrm{~kJ} / \mathrm{kg} \\
& h_{\text {eн } 20}=-12993 \mathrm{~kJ} / \mathrm{kg} \\
& h_{\text {eN2 } 2}=235,3 \mathrm{~kJ} / \mathrm{kg} \\
& h_{\text {eо2 } 2}=212,8 \mathrm{~kJ} / \mathrm{kg} \\
& h_{\text {ehp }}=660,5 \mathrm{~kJ} / \mathrm{kg} \\
& h_{\text {emp }}=652,2 \mathrm{~kJ} / \mathrm{kg}
\end{aligned}
$$

Entropia na entrada:

$$
\begin{aligned}
& S_{\text {eсо2 }}=5,351 \mathrm{~kJ} / \mathrm{kgK} \\
& S_{\text {eн } 20}=11,47 \mathrm{~kJ} / \mathrm{kgK} \\
& S_{e^{2} 2}=7,378 \mathrm{~kJ} / \mathrm{kgK} \\
& S_{e о 2}=6,897 \mathrm{~kJ} / \mathrm{kgK} \\
& S_{e h p}=1,865 \mathrm{~kJ} / \mathrm{kgK} \\
& S_{\text {emp }}=1,881 \mathrm{~kJ} / \mathrm{kgK}
\end{aligned}
$$

\subsection{Equações de balanço}

\section{Balanço de Massa} considerado.

Valor da vazão mássica associada a cada substância presente no volume de controle

$$
\begin{aligned}
& \dot{m}_{e C O 2}=33,59 \mathrm{~kg} / \mathrm{s} \\
& \dot{m}_{\text {eH } 2 O}=27,51 \mathrm{~kg} / \mathrm{s} \\
& \dot{m}_{e N 2}=329,6 \mathrm{~kg} / \mathrm{s} \\
& \dot{m}_{e O 2}=51,29 \mathrm{~kg} / \mathrm{s} \\
& \dot{m}_{e h p}=88,24 \mathrm{~kg} / \mathrm{s} \\
& \dot{m}_{e^{m p}}=4,349 \mathrm{~kg} / \mathrm{s} \\
& \dot{m}_{s i}=\dot{m}_{e i},\left(\mathrm{i}=\mathrm{hp} ; \mathrm{mp} ; \mathrm{CO}_{2} ; \mathrm{H}_{2} \mathrm{O} ; \mathrm{N}_{2} ; \mathrm{O}_{2}\right)
\end{aligned}
$$

Balanço de energia

Da mesma forma como no balanço de massa, não será necessário determinar nenhuma variável pelo balanço de energia para este volume de controle.

\subsection{Condições na saída}

Temperatura na saída:

$$
\begin{aligned}
& T_{s_{g}}=191,9{ }^{\circ} \mathrm{C} \\
& T_{s h p}=222,7^{\circ} \mathrm{C} \\
& T_{s m p}=237,6^{\circ} \mathrm{C}
\end{aligned}
$$

Pressão na saída:

$$
\begin{aligned}
& p_{s h p}=17253 \mathrm{kPa} \\
& p_{s m p}=3541 \mathrm{kPa} \\
& p_{s g}=\frac{103,3}{(1-0,03)^{1} \cdot(1-0,03)^{1} \cdot(1-0,03)^{0}}=109,8 \mathrm{kPa}
\end{aligned}
$$


Entalpia na saída:

$$
\begin{aligned}
& h_{S \mathrm{CO}_{2}}=-8788 \mathrm{~kJ} / \mathrm{kg} \\
& h_{S H 2 O}=-13106 \mathrm{~kJ} / \mathrm{kg} \\
& h_{S N^{2}}=174 \mathrm{~kJ} / \mathrm{kg} \\
& h_{S O 2}=156,3 \mathrm{~kJ} / \mathrm{kg} \\
& h_{S h p}=960,2 \mathrm{~kJ} / \mathrm{kg} \\
& h_{\text {Smp }}=1026 \mathrm{~kJ} / \mathrm{kg}
\end{aligned}
$$

Entropia na saída:

$$
\begin{aligned}
& S_{s \mathrm{CO}_{2}}=5,244 \mathrm{~kJ} / \mathrm{kgK} \\
& S_{S H 2 O}=11,27 \mathrm{~kJ} / \mathrm{kgK} \\
& s_{s N_{2}}=7,272 \mathrm{~kJ} / \mathrm{kgK} \\
& S_{s O_{2}}=6,799 \mathrm{~kJ} / \mathrm{kgK} \\
& S_{s h p}=2,515 \mathrm{~kJ} / \mathrm{kgK} \\
& s_{s m p}=2,678 \mathrm{~kJ} / \mathrm{kgK}
\end{aligned}
$$

\subsection{Análise exergética}

Exergia dos fluxos de massa na entrada:

$$
\begin{aligned}
& e_{e_{i}}=\left(h_{e_{i}}-h_{0 i}\right)-T_{0}\left(S_{e_{i}}-s_{0}\right),\left(\mathrm{i}=\mathrm{hp}, \mathrm{mp}, \mathrm{CO}_{2}, \mathrm{H}_{2} \mathrm{O}, \mathrm{N}_{2}, \mathrm{O}_{2}\right) \\
& e_{\text {есог }}=((-8729)-(-8949))-288,7(5,351-4,826)=68,38 \mathrm{~kJ} / \mathrm{kg} \\
& e_{\text {енго }}=((-12993)-(-13440))-288,7(11,47-10,41)=139,4 \mathrm{~kJ} / \mathrm{kg} \\
& e_{\text {еN2 }}=(235,3-(-9,829))-288,7(7,378-6,799)=77,93 \mathrm{~kJ} / \mathrm{kg} \\
& e_{\text {eог }}=(212,8-(-8,713))-288,7(6,897-6,374)=70,52 \mathrm{~kJ} / \mathrm{kg} \\
& e_{\text {ehp }}=(660,5-65,34)-288,7(1,865-0,2323)=123,8 \mathrm{~kJ} / \mathrm{kg} \\
& e_{\text {emр }}=(652,2-65,34)-288,7(1,881-0,2323)=110,9 \mathrm{~kJ} / \mathrm{kg}
\end{aligned}
$$

Exergia dos fluxos de massa na saída:

$$
\begin{aligned}
& e_{s i}=\left(h_{s i}-h_{0 i}\right)-T_{0}\left(s_{s i}-s 0_{i}\right),\left(\mathrm{i}=\mathrm{hp}, \mathrm{mp}, \mathrm{CO}_{2}, \mathrm{H}_{2} \mathrm{O}, \mathrm{N}_{2}, \mathrm{O}_{2}\right) \\
& e_{\text {sco } 2}=((-8788)-(-8949))-288,7(5,244-4,826)=40,7 \mathrm{~kJ} / \mathrm{kg} \\
& e_{\text {SH } 2 O}=((-13106)-(-13440))-288,7(11,27-10,41)=84,33 \mathrm{~kJ} / \mathrm{kg} \\
& e_{s N_{2}}=(174-(-9,829))-288,7(7,272-6,799)=47,3 \mathrm{~kJ} / \mathrm{kg} \\
& e_{s o 2}=(156,3-(-8,713))-288,7(6,799-6,374)=42,5 \mathrm{~kJ} / \mathrm{kg} \\
& e_{s h p}=(960,2-65,34)-288,7(2,515-0,2323)=235,7 \mathrm{~kJ} / \mathrm{kg} \\
& e_{s m p}=(1026-65,34)-288,7(2,678-0,2323)=254,2 \mathrm{~kJ} / \mathrm{kg}
\end{aligned}
$$

Matéria prima:

$$
\begin{aligned}
& E_{m}=\sum \dot{m}_{i} e_{e i}\left(\mathrm{i}=\mathrm{hp}, \mathrm{mp}, \mathrm{CO}_{2}, \mathrm{H}_{2} \mathrm{O}, \mathrm{N}_{2}, \mathrm{O}_{2}\right) \\
& E_{m}=33,59 \cdot 68,38+27,51 \cdot 139,4+329,6 \cdot 77,93+51,29 \cdot 70,52+88,24 \cdot 123,8+4,349 \cdot 110,9=46840 \mathrm{~kW}
\end{aligned}
$$

Produtos:

$$
\begin{aligned}
& E_{p}=\dot{m}_{s h p} e_{s h p}+\dot{m}_{s m p} e_{s m p} \\
& E_{p}=88,24 \cdot 235,7+4,349 \cdot 254,2=21900 \mathrm{~kW}
\end{aligned}
$$


Perdas:

$E_{l}=\sum \dot{m}_{i} e_{s i},\left(i=\mathrm{CO}_{2}, \mathrm{H}_{2} \mathrm{O}, \mathrm{N}_{2}, \mathrm{O}_{2}\right)$

$E_{l}=33,59 \cdot 40,7+27,51 \cdot 84,33+329,6 \cdot 47,3+51,29 \cdot 42,5=21457 \mathrm{~kW}$

Exergia dissipada:

$\dot{E}_{d}=\dot{E}_{m}-\left(\dot{E}_{p}+\dot{E}_{l}\right)$

$\dot{E}_{d}=46840-(21900+21457)=3483 \mathrm{~kW}$

Eficiência exergética:

$\varepsilon=\frac{\dot{E}_{p}}{\dot{E}_{m}}$

$\varepsilon=\frac{21900}{46840}=0,47$

Eficiência exergética maior:

$\varepsilon^{+}=\frac{\dot{E}_{p}+\dot{E}_{l}}{\dot{E}_{m}}$

$\varepsilon^{+}=\frac{21900+21457}{46840}=0,92$

4.4.1.13 Volume de controle entre os pontos 12 e 13 (Gerador de vapor LP)

4.4.1.13.1 Condições na entrada

Temperatura na entrada:

$T_{e_{g}}=191,9^{\circ} \mathrm{C}$

(Gases)

$T_{e l p}=137,7^{\circ} \mathrm{C}$

(Vapor)

Pressão na entrada:

$p_{e l p}=728,1 \mathrm{kPa}$

$p_{e_{s}}=\frac{103,3}{(1-0,03)^{1} \cdot(1-0,03)^{1} \cdot(1-0,03)^{0}}=109,8 \mathrm{kPa}$

Entalpia na entrada:

$h_{\text {eco2 }}=-8788 \mathrm{~kJ} / \mathrm{kg}$

$h_{\text {eH } 2 O}=-13106 \mathrm{~kJ} / \mathrm{kg}$

$h_{e N 2}=174 \mathrm{~kJ} / \mathrm{kg}$

$h_{\text {eo } 2}=156,3 \mathrm{~kJ} / \mathrm{kg}$

$h_{e l p}=579,7 \mathrm{~kJ} / \mathrm{kg}$

Entropia na entrada:

$S_{\text {eco }}=5,244 \mathrm{~kJ} / \mathrm{kgK}$

Sен $2 \mathrm{O}=11,27 \mathrm{~kJ} / \mathrm{kgK}$

$S_{\text {eN } 2}=7,272 \mathrm{~kJ} / \mathrm{kgK}$

(EES) 


$$
\begin{aligned}
& S_{e o 2}=6,799 \mathrm{~kJ} / \mathrm{kgK} \\
& S_{e l p}=1,715 \mathrm{~kJ} / \mathrm{kgK}
\end{aligned}
$$

\subsection{Equações de balanço}

\section{Balanço de Massa} considerado.

Valor da vazão mássica associada a cada substância presente no volume de controle

$\dot{m}_{\text {eCO2 }}=33,59 \mathrm{~kg} / \mathrm{s}$

$\dot{m}_{\text {eH } 2 O}=27,51 \mathrm{~kg} / \mathrm{s}$

$\dot{m}_{\text {eN } 2}=329,6 \mathrm{~kg} / \mathrm{s}$

$\dot{m}_{e O 2}=51,29 \mathrm{~kg} / \mathrm{s}$

$\dot{m}_{e l p}=111,3 \mathrm{~kg} / \mathrm{s}$

$\dot{m}_{s i}=\dot{m}_{e i},\left(\mathrm{i}=1 \mathrm{p}, \mathrm{CO}_{2} ; \mathrm{H}_{2} \mathrm{O} ; \mathrm{N}_{2} ; \mathrm{O}_{2}\right)$

Balanço de energia

Da mesma forma como no balanço de massa, não será necessário determinar nenhuma variável pelo balanço de energia para este volume de controle.

\subsection{Condições na saída}

Temperatura na saída:

$T_{s_{g}}=161,2^{\circ} \mathrm{C}$

$T_{s l p}=152^{\circ} \mathrm{C}$

$T_{s l s}=152^{\circ} \mathrm{C}$

(Vapor)

Pressão na saída:

$p_{s i p}=501,9 \mathrm{kPa}$

$p_{s l s}=501,9 \mathrm{kPa}$

$p_{s_{g}}=\frac{103,3}{(1-0,03)^{1} \cdot(1-0,03)^{0} \cdot(1-0,03)^{0}}=106,5 \mathrm{kPa}$

Entalpia na saída:

$$
\begin{aligned}
& h_{s \mathrm{CO}_{2}}=-8818 \mathrm{~kJ} / \mathrm{kg} \\
& h_{S \mathrm{H} 2 \mathrm{O}}=-13165 \mathrm{~kJ} / \mathrm{kg} \\
& h_{s{ }^{2} 2}=141,9 \mathrm{~kJ} / \mathrm{kg} \\
& h_{s O_{2}}=126,9 \mathrm{~kJ} / \mathrm{kg} \\
& h_{s l p}=2749 \mathrm{~kJ} / \mathrm{kg} \\
& h_{s l s}=641 \mathrm{~kJ} / \mathrm{kg}
\end{aligned}
$$

Entropia na saída:

$$
\begin{aligned}
& S_{S O_{2}}=5,183 \mathrm{~kJ} / \mathrm{kgK} \\
& S_{\mathrm{H}_{2} \mathrm{O}}=11,16 \mathrm{~kJ} / \mathrm{kgK} \\
& S_{S_{2} 2}=7,209 \mathrm{~kJ} / \mathrm{kgK} \\
& S_{S_{2} 2}=6,741 \mathrm{~kJ} / \mathrm{kgK}
\end{aligned}
$$


$S_{s l p}=6,82 \mathrm{~kJ} / \mathrm{kgK}$
$S_{S l s}=1,862 \mathrm{~kJ} / \mathrm{kgK}$

4.4.1.13.4 Análise exergética

Exergia dos fluxos de massa na entrada:

$e_{e_{i}}=\left(h_{e_{i}}-h_{0_{i}}\right)-\mathrm{T}_{0}\left(\mathrm{Se}_{i}-\mathrm{SO}_{i}\right),\left(i=l p, \mathrm{CO}_{2}, \mathrm{H}_{2} \mathrm{O}, \mathrm{N}_{2}, \mathrm{O}_{2}\right)$

$e_{\text {eсо }}=((-8788)-(-8949))-288,7(5,244-4,826)=40,7 \mathrm{~kJ} / \mathrm{kg}$

(Eq. 2.14)

$e_{\text {ен } 2 \text { о }}=((-13106)-(-13440))-288,7(11,27-10,41)=84,33 \mathrm{~kJ} / \mathrm{kg}$

$e_{\text {eN2 }}=(174-(-9,829))-288,7(7,272-6,799)=47,3 \mathrm{~kJ} / \mathrm{kg}$

$e_{\text {eо } 2}=(156,3-(-8,713))-288,7(6,799-6,374)=42,5 \mathrm{~kJ} / \mathrm{kg}$

$e_{e l p}=(579,7-65,34)-288,7(1,715-0,2323)=86,23 \mathrm{~kJ} / \mathrm{kg}$

Exergia dos fluxos de massa na saída:

$e_{s i}=\left(h_{s i}-h_{0 i}\right)-T_{0}\left(s_{s i}-s_{0}\right),\left(\mathrm{i}=1 \mathrm{p}, 1 \mathrm{~s}, \mathrm{CO}_{2}, \mathrm{H}_{2} \mathrm{O}, \mathrm{N}_{2}, \mathrm{O}_{2}\right)$

(Eq. 2.14)

$e_{s c O 2}=((-8818)-(-8949))-288,7(5,183-4,826)=28,29 \mathrm{~kJ} / \mathrm{kg}$

$e_{\text {SH } 2 O}=((-13165)-(-13440))-288,7(11,16-10,41)=59,15 \mathrm{~kJ} / \mathrm{kg}$

$e_{S_{N} 2}=(141,9-(-9,829))-288,7(7,209-6,799)=33,19 \mathrm{~kJ} / \mathrm{kg}$

$e_{\text {so } 2}=(126,9-(-8,713))-288,7(6,741-6,374)=29,7 \mathrm{~kJ} / \mathrm{kg}$

$e_{s \mid p}=(2749-65,34)-288,7(6,82-0,2323)=781,5 \mathrm{~kJ} / \mathrm{kg}$

$e_{s l s}=(641-65,34)-288,7(1,862-0,2323)=105 \mathrm{~kJ} / \mathrm{kg}$

Matéria prima:

$E_{m}=\sum \dot{m}_{i} e_{e i}\left(i=l p, \mathrm{CO}_{2}, \mathrm{H}_{2} \mathrm{O}, \mathrm{N}_{2}, \mathrm{O}_{2}\right)$

(Eq. 3.75)

$E_{m}=33,59 \cdot 40,7+27,51 \cdot 84,33+329,6 \cdot 47,3+51,29 \cdot 42,5+111,3 \cdot 86,23=31056 \mathrm{~kW}$

Produtos:

$E_{p}=\dot{m}_{s l s} e_{s l s}+\dot{m}_{s p} e_{s l p}$

$E_{p}=3,604 \cdot 781,5+107,7 \cdot 105=14126 \mathrm{~kW}$

(Eq. 3.76)

Perdas:

$E_{l}=\sum \dot{m}_{i} e_{s i},\left(i=\mathrm{CO}_{2}, \mathrm{H}_{2} \mathrm{O}, \mathrm{N}_{2}, \mathrm{O}_{2}\right)$

(Eq. 3.77)

$E_{l}=33,59 \cdot 28,29+27,51 \cdot 59,15+329,6 \cdot 33,19+51,29 \cdot 29,7=15040 \mathrm{~kW}$

Exergia dissipada:

$\dot{E}_{d}=\dot{E}_{m}-\left(\dot{E}_{p}+\dot{E}_{l}\right)$

(Eq. 2.28)

$\dot{E}_{d}=31056-(14126+15040)=1890 \mathrm{~kW}$

Eficiência exergética:

$\varepsilon=\frac{\dot{E}_{p}}{\dot{E}_{m}}$ 
$\varepsilon=\frac{14126}{31056}=0,45$

Eficiência exergética maior:

$$
\begin{aligned}
& \varepsilon^{+}=\frac{\dot{E}_{p}+\dot{E}_{l}}{\dot{E}_{m}} \\
& \varepsilon^{+}=\frac{14126+15040}{31056}=0,94
\end{aligned}
$$

4.4.1.14 Volume de controle entre os pontos 13 e 14 (Economizador LP -passo único)

\subsection{Condições na entrada}

Temperatura na entrada:

$$
T_{e_{g}}=161,2^{\circ} \mathrm{C}
$$

$T_{e l p}=51,44{ }^{\circ} \mathrm{C}$

Pressão na entrada:

$$
\begin{aligned}
& p_{e l p}=749,5 \mathrm{kPa} \\
& p_{e g}=\frac{103,3}{(1-0,03)^{1} \cdot(1-0,03)^{0} \cdot(1-0,03)^{0}}=106,5 \mathrm{kPa}
\end{aligned}
$$

Entalpia na entrada:

$$
\begin{aligned}
& h_{\text {есо } 2}=-8818 \mathrm{~kJ} / \mathrm{kg} \\
& h_{\text {ен } 20}=-13165 \mathrm{~kJ} / \mathrm{kg} \\
& h_{\text {eN2 }}=141,9 \mathrm{~kJ} / \mathrm{kg} \\
& h_{\text {eо } 2}=126,9 \mathrm{~kJ} / \mathrm{kg} \\
& h_{\text {elp }}=216 \mathrm{~kJ} / \mathrm{kg}
\end{aligned}
$$

Entropia na entrada:

$$
\begin{aligned}
& S_{\text {eco2 }}=5,183 \mathrm{~kJ} / \mathrm{kgK} \\
& \text { Sен } 2 \text { о }=11,16 \mathrm{~kJ} / \mathrm{kgK} \\
& S_{e^{N 2}}=7,209 \mathrm{~kJ} / \mathrm{kgK} \\
& S_{e o 2}=6,741 \mathrm{~kJ} / \mathrm{kgK} \\
& s_{e l p}=0,722 \mathrm{~kJ} / \mathrm{kgK}
\end{aligned}
$$

\subsection{Equações de balanço}

\section{Balanço de Massa} considerado.

Valor da vazão mássica associada a cada substância presente no volume de controle

$$
\begin{aligned}
& \dot{m}_{e \mathrm{CO}_{2}}=33,59 \mathrm{~kg} / \mathrm{s} \\
& \dot{m}_{\text {eH } 2 O}=27,51 \mathrm{~kg} / \mathrm{s} \\
& \dot{m}_{e^{N 2}}=329,6 \mathrm{~kg} / \mathrm{s} \\
& \dot{m}_{e O 2}=51,29 \mathrm{~kg} / \mathrm{s}
\end{aligned}
$$




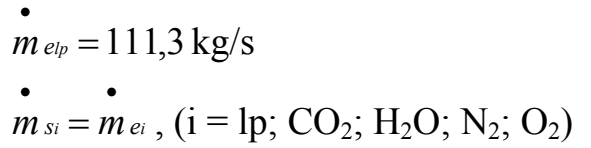

Balanço de energia

Da mesma forma como no balanço de massa, não será necessário determinar nenhuma variável pelo balanço de energia para este volume de controle.

\subsection{Condições na saída}

Temperatura na saída:

$T_{s_{g}}=75,11^{\circ} \mathrm{C}$

(Gases)

$T_{s l p}=137,7^{\circ} \mathrm{C}$

(Vapor)

Pressão na saída:

$p_{s \mid p}=728,1 \mathrm{kPa}$

$p_{s g}=\frac{103,3}{(1-0,03)^{0} \cdot(1-0,03)^{0} \cdot(1-0,03)^{0}}=103,3 \mathrm{kPa}$

Entalpia na saída:

$$
\begin{aligned}
& h_{\mathrm{SCO}_{2}}=-8898 \mathrm{~kJ} / \mathrm{kg} \\
& h_{\text {SH } 2 \mathrm{O}}=-13329 \mathrm{~kJ} / \mathrm{kg} \\
& h_{s N_{2}}=52,22 \mathrm{~kJ} / \mathrm{kg} \\
& h_{\text {so } 2}=46,22 \mathrm{~kJ} / \mathrm{kg} \\
& h_{s \mid p}=579,7 \mathrm{~kJ} / \mathrm{kg}
\end{aligned}
$$

Entropia na saída:

$$
\begin{aligned}
& S_{s C_{2} 2}=4,984 \mathrm{~kJ} / \mathrm{kgK} \\
& S_{S \mathrm{H} 2 \mathrm{O}}=10,75 \mathrm{~kJ} / \mathrm{kgK} \\
& s_{s N 2}=6,988 \mathrm{~kJ} / \mathrm{kgK} \\
& s_{s o 2}=6,542 \mathrm{~kJ} / \mathrm{kgK} \\
& S_{s l p}=1,715 \mathrm{~kJ} / \mathrm{kgK}
\end{aligned}
$$

\subsection{Análise exergética}

Exergia dos fluxos de massa na entrada:

$$
\begin{aligned}
& e_{e_{i}}=\left(h_{e_{i}}-h_{0_{i}}\right)-T_{0}\left(s_{e_{i}}-S_{0}\right),\left(i=l p, \mathrm{CO}_{2}, \mathrm{H}_{2} \mathrm{O}, \mathrm{N}_{2}, \mathrm{O}_{2}\right) \\
& e_{\text {есо2 }}=((-8818)-(-8949))-288,7(5,183-4,826)=28,29 \mathrm{~kJ} / \mathrm{kg} \\
& e_{\text {eн } 20}=((-13165)-(-13440))-288,7(11,16-10,41)=59,15 \mathrm{~kJ} / \mathrm{kg} \\
& e_{e \mathrm{N2}}=(141,9-(-9,829))-288,7(7,209-6,799)=33,19 \mathrm{~kJ} / \mathrm{kg} \\
& e_{e о 2}=(126,9-(-8,713))-288,7(6,741-6,374)=29,7 \mathrm{~kJ} / \mathrm{kg} \\
& e_{e l p}=(216-65,34)-288,7(0,722-0,2323)=9,267 \mathrm{~kJ} / \mathrm{kg}
\end{aligned}
$$

Exergia dos fluxos de massa na saída:

$$
\begin{aligned}
& e_{S i}=\left(h_{s i}-h_{0 i}\right)-T_{0}\left(S_{s i}-s_{0}\right),\left(i=l p, C_{2}, H_{2} O, N_{2}, O_{2}\right) \\
& e_{s C O 2}=((-8898)-(-8949))-288,7(4,984-4,826)=5,793 \mathrm{~kJ} / \mathrm{kg} \\
& e_{S H 2 O}=((-13329)-(-13440))-288,7(10,75-10,41)=12,79 \mathrm{~kJ} / \mathrm{kg} \\
& e_{S{ }_{2} 2}=(52,22-(-9,829))-288,7(6,988-6,799)=7,334 \mathrm{~kJ} / \mathrm{kg}
\end{aligned}
$$


$e_{s o 2}=(46,22-(-8,713))-288,7(6,542-6,374)=6,478 \mathrm{~kJ} / \mathrm{kg}$

$e_{s l p}=(579,7-65,34)-288,7(1,715-0,2323)=86,23 \mathrm{~kJ} / \mathrm{kg}$

Matéria prima:

$E_{m}=\sum \dot{m}_{i} e_{e i}\left(i=l p, \mathrm{CO}_{2}, \mathrm{H}_{2} \mathrm{O}, \mathrm{N}_{2}, \mathrm{O}_{2}\right)$

$E_{m}=33,59 \cdot 28,29+27,51 \cdot 59,15+329,6 \cdot 33,19+51,29 \cdot 29,7+111,3 \cdot 9,267=16072 \mathrm{~kW}$

Produtos:

$$
\begin{aligned}
& E_{p}=\dot{m}_{s l p} e_{s l p} \\
& E_{p}=111,3 \cdot 86,23=9599 \mathrm{~kW}
\end{aligned}
$$

Perdas:

$$
\begin{aligned}
& E_{l}=\sum \dot{m}_{i} e_{s i},\left(i=\mathrm{CO}_{2}, \mathrm{H}_{2} \mathrm{O}, \mathrm{N}_{2}, \mathrm{O}_{2}\right) \\
& E_{l}=33,59 \cdot 5,793+27,51 \cdot 12,79+329,6 \cdot 7,334+51,29 \cdot 6,478=3296 \mathrm{~kW}
\end{aligned}
$$

Exergia dissipada:

$$
\begin{aligned}
& \dot{E}_{d}=\dot{E}_{m}-\left(\dot{E}_{p}+\dot{E}_{l}\right) \\
& \dot{E}_{d}=16072-(9599+3296)=3177 \mathrm{~kW}
\end{aligned}
$$

Eficiência exergética:

$$
\begin{aligned}
& \varepsilon=\frac{\dot{E}_{p}}{\dot{E}_{m}} \\
& \varepsilon=\frac{9599}{16072}=0,60
\end{aligned}
$$

Eficiência exergética maior:

$$
\begin{aligned}
\varepsilon^{+} & =\frac{\dot{E}_{p}+\dot{E}_{l}}{\dot{E}_{m}} \\
\varepsilon^{+} & =\frac{9599+3296}{16072}=0,80
\end{aligned}
$$

\subsubsection{Aquecedor do combustível}

Este trocador de calor difere dos demais localizados no compartimento anteriormente analisado por compreender dois fluidos de trabalho que não possuem nenhuma relação direta com as propriedades dos gases que saem da turbina a gás. Para analisar esse componente serão informadas todas as propriedades termodinâmicas necessárias, inclusive nas condições do ambiente.

4.4.2.1 Condições do ambiente

Fluido de trabalho

Líquido saturado e Gás natural $\left(\mathrm{CH}_{4}\right)$ 
Entalpia para o ambiente

$h_{0 l p}=65,34 \mathrm{~kJ} / \mathrm{kg}$

$h_{0 \mathrm{CH}} 4=-4688 \mathrm{~kJ} / \mathrm{kg}$

Entropia para o ambiente

$s l_{i p}=0,2323 \mathrm{~kJ} / \mathrm{kgK}$

$S_{0 \text { СH } 4}=11,53 \mathrm{~kJ} / \mathrm{kgK}$

4.4.2.2 Condições na entrada

Temperatura na entrada

$T_{e l p}=154,2^{\circ} \mathrm{C}$

$T_{\text {eCH }}=15,56^{\circ} \mathrm{C}$

Entalpia na entrada

$h_{e l p}=650,3 \mathrm{~kJ} / \mathrm{kg}$

$h_{\text {ecH }}=-4688 \mathrm{~kJ} / \mathrm{kg}$

Entropia na entrada

$$
\begin{aligned}
& S_{e l p}=1,884 \mathrm{~kJ} / \mathrm{kgK} \\
& S_{e c H}=9,746 \mathrm{~kJ} / \mathrm{kgK}
\end{aligned}
$$

4.4.2.3 Balanço de massa

$$
\begin{aligned}
& \dot{m}_{e l p}=15,12 \mathrm{~kg} / \mathrm{s} \\
& \dot{m}_{e C H 4}=8,693 \mathrm{~kg} / \mathrm{s} \\
& \dot{m}_{s l p}=\dot{m}_{e l p} \\
& \dot{m}_{S C H 4}=\dot{m}_{e C H 4}
\end{aligned}
$$

4.4.2.4 Balanço de energia

$$
\dot{m}_{e l p} h_{e l p}+\dot{m}_{e C H 4} h_{e C H}=\dot{m}_{s l p} h_{s l p}+\dot{m}_{s \mathrm{CH} 4} h_{S C H}
$$

Neste caso, como todas as propriedades estão disponíveis, não será calculada nenhuma variável a partir do balanço de energia.

\subsubsection{Condições na entrada}

Temperatura na saída

$T_{s l p}=112,3^{\circ} \mathrm{C}$

$T_{\text {SCH } 4}=147,9^{\circ} \mathrm{C}$

Entalpia na saída

$$
h_{s l p}=471,7 \mathrm{~kJ} / \mathrm{kg}
$$

$h_{\text {SCH }}=-4371 \mathrm{~kJ} / \mathrm{kg}$

Entropia na saída

$$
\begin{aligned}
& S_{S l p}=1,444 \mathrm{~kJ} / \mathrm{kgK} \\
& S_{s C H}=10,65 \mathrm{~kJ} / \mathrm{kgK}
\end{aligned}
$$


4.4.2.6 Análise exergética

Exergia dos fluxos de massa na entrada:

$e_{e_{i}}=\left(h_{e_{i}}-h_{0_{i}}\right)-T_{0}\left(S_{e_{i}}-s 0_{i}\right)\left(i=l p, \mathrm{CH}_{4}\right)$

$e_{e l_{p}}=(650,3-65,34)-288,7(1,884-0,2323)=108,1 \mathrm{~kJ} / \mathrm{kg}$

$e_{\text {есн } 4}=((-4688)-(-4688))-288,7(9,746-11,53)=515,2 \mathrm{~kJ} / \mathrm{kg}$

Exergia dos fluxos de massa na saída:

$e_{s i}=\left(h_{s i}-h_{0 i}\right)-T_{0}\left(s_{s i}-s 0_{i}\right)\left(i=l p, C H_{4}\right)$

$e_{s i p}=(471,7-65,34)-288,7(1,444-0,2323)=56,56 \mathrm{~kJ} / \mathrm{kg}$

(Eq. 2.14)

$e_{S C H 4}=((-4371)-(-4688))-288,7(10,65-11,53)=572,6 \mathrm{~kJ} / \mathrm{kg}$

Matéria prima:

$E_{m}=\dot{m}_{\text {elp }} e_{e l p}+\dot{m}_{\text {есн } 4 e_{e c H} 4}$

$E_{m}=15,12 \cdot 108,1+8,693 \cdot 515,2=6113 \mathrm{~kW}$

(Eq. 3.75)

Produtos:

$E_{p}=\dot{m}_{\text {SCH } 4} e_{s C H}$

$E_{p}=8,693 \cdot 572,6=4978 \mathrm{~kW}$

(Eq. 3.76)

Perdas:

$E_{l}=\dot{m}_{s l p} e_{s l p}$

$E_{l}=15,12 \cdot 56,56=855,2 \mathrm{~kW}$

(Eq. 3.77)

Exergia dissipada:

$\dot{E}_{d}=\dot{E}_{m}-\left(\dot{E}_{p}+\dot{E}_{l}\right)$

$\dot{E}_{d}=6113-(4978+855,2)=279,8 \mathrm{~kW}$

Eficiência exergética:

$\varepsilon=\frac{\dot{E}_{p}}{\dot{E}_{m}}$

$\varepsilon=\frac{4978}{6113}=0,81$

Eficiência exergética maior:

$$
\begin{aligned}
& \varepsilon^{+}=\frac{\dot{E}_{p}+\dot{E}_{l}}{\dot{E}_{m}} \\
& \varepsilon^{+}=\frac{4978+855,2}{6113}=0,95
\end{aligned}
$$

\subsubsection{Turbina a vapor}


Conforme informado anteriormente, no ciclo de Uruguaiana existem três turbinas a vapor sendo uma para cada nível de pressão. Para este componente será analisada cada turbina separadamente.

4.4.3.1 Condições do ambiente

Fluido de trabalho

Vapor

Entalpia para o ambiente

$h_{0 h p}=65,34 \mathrm{~kJ} / \mathrm{kg}$

Entropia para o ambiente

$s_{0 h p}=0,2323 \mathrm{~kJ} / \mathrm{kgK}$

4.4.3.2 Turbina de alta pressão

\subsection{Condições na entrada}

Temperatura na entrada

$T_{e h p}=526,6^{\circ} \mathrm{C}$

Pressão na entrada

$p_{\text {ehp }}=15623 \mathrm{kPa}$

Entalpia na entrada

$h_{\text {ehp }}=3378 \mathrm{~kJ} / \mathrm{kg}$

Entropia na entrada

$S_{e h p}=6,415 \mathrm{~kJ} / \mathrm{kgK}$

4.4.3.2.2 Balanço de massa

$\dot{m}_{e h p}=176,5 \mathrm{~kg} / \mathrm{s}$

$\dot{m}_{\text {shp }}=\dot{m}_{\text {ehp }}$

4.4.3.2.3 Condições na saída

Temperatura na saída

$T_{\text {shp }}=321,7^{\circ} \mathrm{C}$

Pressão na saída

$p_{s h p}=3641 \mathrm{kPa}$

Entalpia na saída

$h_{s h p}=3029 \mathrm{~kJ} / \mathrm{kg}$

Entropia na saída

$S_{s h p}=6,518 \mathrm{~kJ} / \mathrm{kgK}$

4.4.3.2.4 Balanço de energia

$\dot{m}_{e h p} h_{e h p}=\dot{W}_{t v}+\dot{m}_{S h p} h_{S h p}$ 
Agora pode-se calcular a potência fornecida pela turbina a vapor de alta pressão

$$
\dot{W}_{t v}=176,5 \cdot(3378-3029)=61500 \mathrm{~kW}
$$

\subsection{Análise exergética}

Exergia dos fluxos de massa na entrada:

$$
\begin{aligned}
& e_{e h p}=\left(h_{e h p}-h_{0 h p}\right)-T_{0}\left(S_{e h p}-S 0_{h p}\right) \\
& e_{e h p}=(3378-65,34)-288,7(6,415-0,2323)=1527 \mathrm{~kJ} / \mathrm{kg}
\end{aligned}
$$

Exergia dos fluxos de massa na saída:

$$
\begin{aligned}
& e_{s h p}=\left(h_{S h p}-h_{0 h p}\right)-T_{0}\left(S_{s h p}-S_{0 h p}\right) \\
& e_{S h p}=(3029-65,34)-288,7(6,518-0,2323)=1149 \mathrm{~kJ} / \mathrm{kg}
\end{aligned}
$$

Matéria prima:

$$
\begin{aligned}
& E_{m}=\dot{m}_{e_{h p} e_{e h p}} \\
& E_{m}=176,5 \cdot 1527=269531 \mathrm{~kW}
\end{aligned}
$$

Produtos:

$$
\begin{aligned}
& E_{p}=\dot{W}_{t v} \\
& E_{p}=61500 \mathrm{~kW}
\end{aligned}
$$

Perdas:

$$
\begin{aligned}
& E_{l}=\dot{m}_{s h p} e_{S h p} \\
& E_{l}=176,5 \cdot 1149=202783 \mathrm{~kW}
\end{aligned}
$$

Exergia dissipada:

$$
\begin{aligned}
& \dot{E}_{d}=\dot{E}_{m}-\left(\dot{E}_{p}+\dot{E}_{l}\right) \\
& \dot{E}_{d}=269531-(61500+202783)=5249 \mathrm{~kW}
\end{aligned}
$$

Eficiência exergética:

$$
\begin{aligned}
& \varepsilon=\frac{\dot{E}_{p}}{\dot{E}_{m}} \\
& \varepsilon=\frac{61500}{269531}=0,23
\end{aligned}
$$

Eficiência exergética maior:

$$
\begin{aligned}
\varepsilon^{+} & =\frac{\dot{E}_{p}+\dot{E}_{l}}{\dot{E}_{m}} \\
\varepsilon^{+} & =\frac{61500+202783}{269531}=0,98
\end{aligned}
$$


4.4.3.3 Turbina de média pressão

\subsection{Condições na entrada}

Temperatura na entrada

$T_{\text {emp }}=537,1^{\circ} \mathrm{C}$

Pressão na entrada

$p_{\text {emp }}=3203 \mathrm{kPa}$

Entalpia na entrada

$h_{\text {emp }}=3538 \mathrm{~kJ} / \mathrm{kg}$

Entropia na entrada

$S_{\text {emp }}=7,307 \mathrm{~kJ} / \mathrm{kgK}$

4.4.3.3.2 Balanço de massa

$$
\begin{aligned}
& \dot{m}_{\text {emp }}=179,4 \mathrm{~kg} / \mathrm{s} \\
& \dot{m}_{\text {smp }}=\dot{m}_{\text {emp }}
\end{aligned}
$$

\subsection{Condições na saída}

Temperatura na saída

$T_{\text {smp }}=360,9^{\circ} \mathrm{C}$

Pressão na saída

$p_{s m p}=976,3 \mathrm{kPa}$

Entalpia na saída

$h_{\text {smp }}=3181 \mathrm{~kJ} / \mathrm{kg}$

Entropia na saída

$$
S_{\text {Smp }}=7,349 \mathrm{~kJ} / \mathrm{kgK}
$$

4.4.3.3.4 Balanço de energia

$\dot{m}_{e_{m p}} h_{e m p}=\dot{W}_{t v}+\dot{m}_{s m p} h_{s m p}$

Agora pode-se calcular a potência fornecida pela turbina a vapor de alta pressão

$\dot{W}_{t v}=179,4 \cdot(3538-3181)=64045 \mathrm{~kW}$

\subsection{Análise exergética}

Exergia dos fluxos de massa na entrada:

$e_{\text {emp }}=\left(h_{\text {emp }}-h_{0 m p}\right)-T_{0}\left(s_{\text {mp }}-s_{0 m p}\right)$

$e_{e h p}=(3538-65,34)-288,7(7,307-0,2323)=1430 \mathrm{~kJ} / \mathrm{kg}$

Exergia dos fluxos de massa na saída:

$e_{s m p}=\left(h_{s m p}-h_{0 m p}\right)-T_{0}\left(s_{s m p}-s_{0 m p}\right)$ 
$e_{s m p}=(3181-65,34)-288,7(7,349-0,2323)=1061 \mathrm{~kJ} / \mathrm{kg}$

Matéria prima:

$$
\begin{aligned}
& E_{m}=\dot{m}_{e_{m p} e_{e_{m p}}} \\
& E_{m}=179,4 \cdot 1430=256625 \mathrm{~kW}
\end{aligned}
$$

Produtos:

$$
\begin{aligned}
& E_{p}=\dot{W}_{t v} \\
& E_{p}=64045 \mathrm{~kW}
\end{aligned}
$$

Perdas:

$$
\begin{aligned}
& E_{l}=\dot{m}_{s_{m p} e_{S m p}} \\
& E_{l}=179,4 \cdot 1061=190406 \mathrm{~kW}
\end{aligned}
$$

Exergia dissipada:

$$
\begin{aligned}
& \dot{E}_{d}=\dot{E}_{m}-\left(\dot{E}_{p}+\dot{E}_{l}\right) \\
& \dot{E}_{d}=256625-(64045+190406)=2174 \mathrm{~kW}
\end{aligned}
$$

Eficiência exergética:

$$
\begin{aligned}
\varepsilon & =\frac{\dot{E}_{p}}{\dot{E}_{m}} \\
\varepsilon & =\frac{64045}{256625}=0,25
\end{aligned}
$$

Eficiência exergética maior:

$$
\begin{aligned}
& \varepsilon^{+}=\frac{\dot{E}_{p}+\dot{E}_{l}}{\dot{E}_{m}} \\
& \varepsilon^{+}=\frac{64045+190406}{256625}=0,99
\end{aligned}
$$

\subsubsection{Turbina de baixa pressão}

A turbina a vapor de baixa pressão, difere das outras demais por apresentar dois fluxos de vapor em condições de pressão e temperatura distintas para a entrada da turbina. O vapor que sai da turbina em média pressão une-se ao vapor fornecido em baixa pressão antes de ser expandido na turbina de baixa pressão. A seguir serão descritos cada condição de fornecimento do vapor de média e baixa pressão.

\subsection{Condições na entrada}

Temperatura na entrada

$T_{e m p}=360,9^{\circ} \mathrm{C}$

$T_{e l p}=304,8^{\circ} \mathrm{C}$

Pressão na entrada 
$p_{\text {emp }}=976,3 \mathrm{kPa}$

$p_{e l p}=437,1 \mathrm{kPa}$

Entalpia na entrada

$h_{\text {emp }}=3181 \mathrm{~kJ} / \mathrm{kg}$

$h_{e l p}=3075 \mathrm{~kJ} / \mathrm{kg}$

Entropia na entrada

$S_{e m p}=7,349 \mathrm{~kJ} / \mathrm{kgK}$

$S_{e l p}=7,541 \mathrm{~kJ} / \mathrm{kgK}$

4.4.3.4.2 Balanço de massa

$$
\begin{aligned}
& \dot{m}_{e_{m p}}=184,2 \mathrm{~kg} / \mathrm{s} \\
& \dot{m}_{e l p}=7,207 \mathrm{~kg} / \mathrm{s} \\
& \dot{m}_{e^{m p}}+\dot{m}_{e l p}=\dot{m}_{s l p} \\
& \dot{m}_{s l p}=184,2+7,207=191,407
\end{aligned}
$$

\subsection{Condições na saída}

Temperatura na saída

$T_{s l p}=38,94{ }^{\circ} \mathrm{C}$

Pressão na saída

$p_{s l p}=6,971 \mathrm{kPa}$

Entalpia na saída

$h_{s \mid p}=2405 \mathrm{~kJ} / \mathrm{kg}$

Entropia na saída

$s_{s l p}=8,275 \mathrm{~kJ} / \mathrm{kgK}$

4.4.3.4.4 Balanço de energia

$\dot{m}_{e l p} h_{e l p}+\dot{m}_{e m p} h_{e m p}=\dot{W}_{t v}+\dot{m}_{s l p} h_{s l p}$

Agora pode-se calcular a potência fornecida pela turbina a vapor de alta pressão

$\dot{W}_{t v}=184,2 \cdot 3181+7,207 \cdot 3075-191,407 \cdot 2405=147793 \mathrm{~kW}$

\subsection{Análise exergética}

Exergia dos fluxos de massa na entrada:

$e_{e_{i}}=\left(h_{e_{i}}-h_{0 i}\right)-T_{0}\left(s_{e_{i}}-s 0_{i}\right)(i=m p, l p)$

$e_{e m p}=(3181-65,34)-288,7(7,349-0,2323)=1061 \mathrm{~kJ} / \mathrm{kg}$

$e_{e l p}=(3075-65,34)-288,7(7,541-0,2323)=900,1 \mathrm{~kJ} / \mathrm{kg}$

Exergia dos fluxos de massa na saída:

$e_{s l p}=\left(h_{s l p}-h_{0 l p}\right)-T_{0}\left(s_{s l p}-s 0_{l p}\right)$ 
$e_{s l p}=(2405-65,34)-288,7(8,275-0,2323)=17,43 \mathrm{~kJ} / \mathrm{kg}$

Matéria prima:

$E_{m}=\dot{m}_{e_{p l} e_{e l p}}+\dot{m}_{e_{m p} e_{e m p}}$

$E_{m}=184,2 \cdot 1061+7,207 \cdot 900,1=201903 \mathrm{~kW}$

(Eq. 3.61)

Produtos:

$E_{p}=\dot{W}_{t v}$

$E_{p}=147793 \mathrm{~kW}$

Perdas:

$E_{l}=\dot{m}_{s l p} e_{s l p}$

$E_{l}=191,4 \cdot 17,43=3336 \mathrm{~kW}$

Exergia dissipada:

$\dot{E}_{d}=\dot{E}_{m}-\left(\dot{E}_{p}+\dot{E}_{l}\right)$

$\dot{E}_{d}=201903-(147793+3336)=50774 \mathrm{~kW}$

Eficiência exergética:

$$
\begin{aligned}
& \varepsilon=\frac{\dot{E}_{p}}{\dot{E}_{m}} \\
& \varepsilon=\frac{147793}{201903}=0,73
\end{aligned}
$$

Eficiência exergética maior:

$$
\begin{aligned}
\varepsilon^{+} & =\frac{\dot{E}_{p}+\dot{E}_{l}}{\dot{E}_{m}} \\
\varepsilon^{+} & =\frac{147793+3336}{201903}=0,74
\end{aligned}
$$

\subsubsection{Condensador}

Este componente apresenta uma grande troca de calor entre o vapor de saída da turbina de alta pressão e a água de alimentação que é resfriada em uma torre de resfriamento.

\subsubsection{Condições do ambiente}

Fluido de trabalho

Vapor de saída da turbina de baixa pressão e água

Entalpia para o ambiente

$$
\begin{aligned}
& h_{0 l p}=65,34 \mathrm{~kJ} / \mathrm{kg} \\
& h_{0 \text { н } 2 \text { O }}=65,38 \mathrm{~kJ} / \mathrm{kg}
\end{aligned}
$$

Entropia para o ambiente 
$s 0_{l p}=0,2323 \mathrm{~kJ} / \mathrm{kgK}$
$s_{0{ }^{2 O} \mathrm{O}}=0,2324 \mathrm{~kJ} / \mathrm{kgK}$

4.4.4.2 Condições na entrada

Temperatura na entrada

$T_{e l p}=38,94{ }^{\circ} \mathrm{C}$

$T_{\text {eH } 2 \mathrm{O}}=21,28^{\circ} \mathrm{C}$

Entalpia na entrada

$h_{e l p}=2571 \mathrm{~kJ} / \mathrm{kg}$

$h_{\text {eH } 2 O}=89,21 \mathrm{~kJ} / \mathrm{kg}$

Entropia na entrada

$S_{e l p}=8,275 \mathrm{~kJ} / \mathrm{kgK}$

Sен 2 о $=0,3146 \mathrm{~kJ} / \mathrm{kgK}$

4.4.4.3 Balanço de massa

$$
\begin{aligned}
& \dot{m}_{e l p}=191,4 \mathrm{~kg} / \mathrm{s} \\
& \dot{m}_{e H 2 O}=7345 \mathrm{~kg} / \mathrm{s} \\
& \dot{m}_{S l p}=\dot{m}_{e l p} \\
& \dot{m}_{S H 2 O}=\dot{m}_{\text {eH } 2 O}
\end{aligned}
$$

4.4.4.4 Balanço de energia

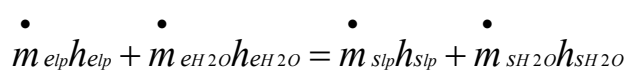

(Eq. 3.80)

Neste caso, como todas as propriedades estão disponíveis, não será calculada nenhuma variável a partir do balanço de energia.

4.4.4.5 Condições na entrada

Temperatura na saída

$T_{s l p}=38,89^{\circ} \mathrm{C}$

$T_{S H 2 O}=34,17^{\circ} \mathrm{C}$

Entalpia na saída

$h_{s l p}=162,9 \mathrm{~kJ} / \mathrm{kg}$

$h_{\text {sH } 2 O}=143 \mathrm{~kJ} / \mathrm{kg}$

Entropia na saída

$$
s_{s l p}=0,5574 \mathrm{~kJ} / \mathrm{kgK}
$$

$S_{S H 2 O}=0,4935 \mathrm{~kJ} / \mathrm{kgK}$

4.4.4.6 Análise exergética

Exergia dos fluxos de massa na entrada:

$$
\begin{aligned}
& e_{e_{i}}=\left(h_{e_{i}}-h_{0_{i}}\right)-T_{0}\left(S_{e_{i}}-S 0_{i}\right)\left(i=l p, H_{2} O\right) \\
& e_{e_{l p}}=(2571-65,34)-288,7(8,275-0,2323)=184,1 \mathrm{~kJ} / \mathrm{kg}
\end{aligned}
$$


$e_{\text {ен } 2 \text { О }}=(89,21-65,38)-288,7(0,3146-0,2324)=0,09788 \mathrm{~kJ} / \mathrm{kg}$

Exergia dos fluxos de massa na saída:

$$
\begin{aligned}
& e_{s i}=\left(h_{s i}-h_{0 i}\right)-T_{0}\left(s_{s i}-s_{0}\right)\left(i=l p, H_{2} O\right) \\
& e_{s l p}=(162,9-65,34)-288,7(0,5574-0,2323)=3,649 \mathrm{~kJ} / \mathrm{kg} \\
& e_{s H 2 O}=(143-65,38)-288,7(0,4935-0,2324)=2,271 \mathrm{~kJ} / \mathrm{kg}
\end{aligned}
$$

Matéria prima:

$$
\begin{aligned}
& E_{m}=\dot{m}_{e_{l p} e_{e l p}}+\dot{m}_{\text {енгоеен } 20} \\
& E_{m}=191,4 \cdot 184,1+7345 \cdot 0,09788=35942 \mathrm{~kW}
\end{aligned}
$$

Produtos:

$$
\begin{aligned}
& E_{p}=\dot{m}_{S H 2 O} e_{S H 2 O} \\
& E_{p}=7345 \cdot 2,271=16681 \mathrm{~kW}
\end{aligned}
$$

Perdas:

$$
\begin{aligned}
& E_{l}=\dot{m}_{s l p} e_{s l p} \\
& E_{l}=191,4 \cdot 3,649=698,4 \mathrm{~kW}
\end{aligned}
$$

Exergia dissipada:

$$
\begin{aligned}
& \dot{E}_{d}=\dot{E}_{m}-\left(\dot{E}_{p}+\dot{E}_{l}\right) \\
& \dot{E}_{d}=35942-(16681+698,4)=18562 \mathrm{~kW}
\end{aligned}
$$

Eficiência exergética:

$$
\begin{aligned}
& \varepsilon=\frac{\dot{E}_{p}}{\dot{E}_{m}} \\
& \varepsilon=\frac{16681}{35942}=0,46
\end{aligned}
$$

Eficiência exergética maior:

$$
\begin{aligned}
\varepsilon^{+} & =\frac{\dot{E}_{p}+\dot{E}_{l}}{\dot{E}_{m}} \\
\varepsilon^{+} & =\frac{16681+698,4}{35942}=0,48
\end{aligned}
$$

\subsubsection{Bomba de água}

Para este componente a análise será restrita às bombas localizadas após a saída de líquido saturado do gerador de vapor em baixa pressão e a bomba localizada após o condensador. Serão analisadas quatro bombas que bombeiam o liquido saturado para os geradores de alta e média pressão, a bomba para líquido saturado que aquece o gás natural antes desse entrar no combustor e a bomba que bombeia o condensado que sai do condensador. Apesar de haverem outras bombas no 
ciclo, essas quatro são as que apresentam maior representatividade em relação à eficiência exergética.

4.4.5.1 Condições do ambiente

Fluido de trabalho

Líquido saturado

Entalpia para o ambiente

$h_{0 l s}=65,34 \mathrm{~kJ} / \mathrm{kg}$

Entropia para o ambiente

$s_{0 l s}=0,2323 \mathrm{~kJ} / \mathrm{kgK}$

4.4.5.2 Bomba de média pressão

\subsection{Condições na entrada}

Temperatura na entrada

$T_{e l p}=152^{\circ} \mathrm{C}$

Pressão na entrada

$p_{e l p}=501,9 \mathrm{kPa}$

Entalpia na entrada

$h_{e l p}=641 \mathrm{~kJ} / \mathrm{kg}$

Entropia na entrada

$S_{e l p}=1,862 \mathrm{~kJ} / \mathrm{kgK}$

4.4.5.2.2 Balanço de massa

$\dot{m}_{\text {elp }}=4,349 \mathrm{~kg} / \mathrm{s}$

$\dot{m}_{s m p}=\dot{m}_{e l p}$

4.4.5.2.3 Condições na saída

Temperatura na saída

$T_{s m p}=154,2^{\circ} \mathrm{C}$

Pressão na saída

$p_{\text {smp }}=3551 \mathrm{kPa}$

Entalpia na saída

$h_{\text {smp }}=650,3 \mathrm{~kJ} / \mathrm{kg}$

Entropia na saída

$S_{s m p}=1,884 \mathrm{~kJ} / \mathrm{kgK}$

4.4.5.2.4 Balanço de energia 
A potência consumida pela bomba será calculada através da equação (3.88) onde foi admitida uma eficiência de 0,9 para a bomba. A densidade considerada será a do líquido saturado à temperatura de entrada.

$$
\begin{aligned}
\rho_{m p} & =915,2 \mathrm{~kg} / \mathrm{m}^{3} \\
\eta_{b b} & =\frac{\dot{m} l\left(p_{s l}-p_{e l}\right)}{\dot{W}_{p}} \\
\dot{W}_{p} & =\frac{4,349 \cdot(3551-501,9)}{0,9 \cdot 915,2}=16,1 \mathrm{~kW}
\end{aligned}
$$

\subsection{Análise exergética}

Exergia dos fluxos de massa na entrada:

$e_{e}=\left(h_{e l p}-h_{0 l s}\right)-T_{0}\left(S_{e l p}-S_{0 l s}\right)$

$e_{e l p}=(641-65,34)-288,7(1,862-0,2323)=105 \mathrm{~kJ} / \mathrm{kg}$

Exergia dos fluxos de massa na saída:

$$
\begin{aligned}
& e_{s m p}=\left(h_{s l p}-h_{0 l s}\right)-T_{0}\left(s_{s m p}-s_{0 l s}\right) \\
& e_{s m p}=(650,3-65,34)-288,7(1,884-0,2323)=108,1 \mathrm{~kJ} / \mathrm{kg}
\end{aligned}
$$

Matéria prima:

$$
\begin{aligned}
& E_{m}=\dot{m}_{e^{l} e_{e l p}}+\dot{W}_{b} \\
& E_{m}=4,349 \cdot 105+16,1=472,8 \mathrm{~kW}
\end{aligned}
$$

Produtos:

$$
\begin{aligned}
& E_{p}=\dot{m}_{s m p} e_{s m p} \\
& E_{p}=4,349 \cdot 108,1=470 \mathrm{~kW}
\end{aligned}
$$

Perdas:

Não há perdas neste componente.

Exergia dissipada:

$$
\begin{aligned}
& \dot{E}_{d}=\dot{E}_{m}-\left(\dot{E}_{p}+\dot{E}_{l}\right) \\
& \dot{E}_{d}=472,8-(470+0)=2,8 \mathrm{~kW}
\end{aligned}
$$

Eficiência exergética:

$$
\begin{aligned}
& \varepsilon=\frac{\dot{E}_{p}}{\dot{E}_{m}} \\
& \varepsilon=\frac{470}{472,8}=0,99
\end{aligned}
$$

Eficiência exergética maior: 


$$
\begin{aligned}
\varepsilon^{+} & =\frac{\dot{E}_{p}+\dot{E}_{l}}{\dot{E}_{m}} \\
\varepsilon^{+} & =\frac{470+0}{472,8}=0,99
\end{aligned}
$$

4.4.5.3 Bomba de alta pressão

\subsection{Condições na entrada}

Temperatura na entrada

$T_{e l p}=152{ }^{\circ} \mathrm{C}$

Pressão na entrada

$p_{e l p}=501,9 \mathrm{kPa}$

Entalpia na entrada

$h_{e l p}=641 \mathrm{~kJ} / \mathrm{kg}$

Entropia na entrada

$S_{\text {elp }}=1,862 \mathrm{~kJ} / \mathrm{kgK}$

\subsection{Balanço de massa}

$$
\begin{aligned}
& \dot{m}_{\text {elp }}=88,24 \mathrm{~kg} / \mathrm{s} \\
& \dot{m}_{\text {shp }}=\dot{m}_{\text {elp }}
\end{aligned}
$$

\subsection{Condições na saída}

Temperatura na saída

$T_{\text {shp }}=154,1^{\circ} \mathrm{C}$

Pressão na saída

$p_{s h p}=17432 \mathrm{kPa}$

Entalpia na saída

$$
h_{s h p}=660,5 \mathrm{~kJ} / \mathrm{kg}
$$

Entropia na saída

$$
S_{S h p}=1,865 \mathrm{~kJ} / \mathrm{kgK}
$$

\subsection{Balanço de energia}

A potência consumida pela bomba será calculada através da equação (3.88) onde foi admitida uma eficiência de 0,9 para a bomba. A densidade considerada será a do líquido saturado à temperatura de entrada.

$$
\begin{aligned}
\rho_{h p} & =915,2 \mathrm{~kg} / \mathrm{m}^{3} \\
\eta_{b b} & =\frac{\dot{m} l\left(p_{s l}-p_{e l}\right)}{\rho \dot{W_{p}}}
\end{aligned}
$$




$$
\dot{W}_{p}=\frac{88,24 \cdot(17432-501,9)}{0,9 \cdot 915,2}=1814 \mathrm{~kW}
$$

\subsection{Análise exergética}

Exergia dos fluxos de massa na entrada:

$$
\begin{aligned}
& e_{e}=\left(h_{e l p}-h_{0 l s}\right)-T_{0}\left(s_{e l p}-s_{0 l s}\right) \\
& e_{e l p}=(641-65,34)-288,7(1,862-0,2323)=105 \mathrm{~kJ} / \mathrm{kg}
\end{aligned}
$$

Exergia dos fluxos de massa na saída:

$$
\begin{aligned}
& e_{s h p}=\left(h_{s h p}-h_{0 l s}\right)-T_{0}\left(S_{s h p}-S 0_{l s}\right) \\
& e_{s h p}=(660,5-65,34)-288,7(1,865-0,2323)=123,8 \mathrm{~kJ} / \mathrm{kg}
\end{aligned}
$$

Matéria prima:

$$
\begin{aligned}
& E_{m}=\dot{m}_{e_{1 p} e_{e l p}+\dot{W}_{b}} \\
& E_{m}=88,24 \cdot 105+1814=11079 \mathrm{~kW}
\end{aligned}
$$

Produtos:

$$
\begin{aligned}
& E_{p}=\dot{\bar{m}}_{s h p} e_{s h p} \\
& E_{p}=88,24 \cdot 123,8=10922 \mathrm{~kW}
\end{aligned}
$$

Perdas:

Não há perdas neste componente.

Exergia dissipada:

$$
\begin{aligned}
& \dot{E}_{d}=\dot{E}_{m}-\left(\dot{E}_{p}+\dot{E}_{l}\right) \\
& \dot{E}_{d}=11079-(10922+0)=156,7 \mathrm{~kW}
\end{aligned}
$$

Eficiência exergética:

$$
\begin{aligned}
& \varepsilon=\frac{\dot{E}_{p}}{\dot{E}_{m}} \\
& \varepsilon=\frac{10922}{11079}=0,98
\end{aligned}
$$

Eficiência exergética maior:

$$
\begin{aligned}
& \varepsilon^{+}=\frac{\dot{E}_{p}+\dot{E}_{l}}{\dot{E}_{m}} \\
& \varepsilon^{+}=\frac{10922+0}{11079}=0,98
\end{aligned}
$$

4.4.5.4 Bomba do líquido saturado para aquecimento do combustível

\subsection{Condições na entrada}


Temperatura na entrada

$T_{e l p}=152{ }^{\circ} \mathrm{C}$

Pressão na entrada

$p_{e l p}=501,9 \mathrm{kPa}$

Entalpia na entrada

$h_{e l p}=641 \mathrm{~kJ} / \mathrm{kg}$

Entropia na entrada

$S_{e l p}=1,862 \mathrm{~kJ} / \mathrm{kgK}$

4.4.5.4.2 Balanço de massa

$\dot{m}_{\text {elp }}=15,12 \mathrm{~kg} / \mathrm{s}$

$\dot{m}_{s h p}=\dot{m}_{e l p}$

\subsection{Condições na saída}

Temperatura na saída

$T_{\text {smpcb }}=152^{\circ} \mathrm{C}$

Pressão na saída

$p_{\text {smpcb }}=770,8 \mathrm{kPa}$

Entalpia na saída

$h_{\text {smpcb }}=641 \mathrm{~kJ} / \mathrm{kg}$

Entropia na saída

$S_{\text {smpcb }}=1,862 \mathrm{~kJ} / \mathrm{kgK}$

\subsection{Balanço de energia}

A potência consumida pela bomba será calculada através da equação (3.88) onde foi admitida uma eficiência de 0,9 para a bomba. A densidade considerada será a do líquido saturado à temperatura de entrada.

$$
\begin{aligned}
& \rho_{l p}=915,2 \mathrm{~kg} / \mathrm{m}^{3} \\
& \eta_{b b}=\frac{\dot{m}_{l}\left(p_{s l}-p_{e l}\right)}{\frac{\dot{W}_{p}}{\rho W^{2}}} \\
& \dot{W}_{p}=\frac{15,12 \cdot(770,8-501,9)}{0,9 \cdot 915,2}=4,93 \mathrm{~kW}
\end{aligned}
$$

\subsection{Análise exergética}

Exergia dos fluxos de massa na entrada:

$e_{e}=\left(h_{e l p}-h_{0 l s}\right)-T_{0}\left(S_{e l p}-S 0_{l s}\right)$

$e_{e l p}=(641-65,34)-288,7(1,862-0,2323)=105 \mathrm{~kJ} / \mathrm{kg}$

Exergia dos fluxos de massa na saída: 
$e_{\text {smpcb }}=\left(h_{\text {smpcb }}-h_{0 l s}\right)-T_{0}\left(s_{\text {smpec }}-S 0 l s\right)$

$e_{\text {smpec }}=(641-65,34)-288,7(1,862-0,2323)=105 \mathrm{~kJ} / \mathrm{kg}$

Matéria prima:

$E_{m}=\dot{m}_{e l p} e_{e l p}+\dot{W}_{b}$

$E_{m}=15,12 \cdot 105+4,93=1593 \mathrm{~kW}$

Produtos:

$E_{p}=\dot{\bar{m}}_{\text {smpcce }}$ Smppc $_{\text {spe }}$
$E_{p}=15,12 \cdot 105=1588 \mathrm{~kW}$

Perdas:

Não há perdas neste componente.

Exergia dissipada:

$\dot{E}_{d}=\dot{E}_{m}-\left(\dot{E}_{p}+\dot{E}_{l}\right)$

$\dot{E}_{d}=1593-(1588+0)=5 \mathrm{~kW}$

Eficiência exergética:

$\varepsilon=\frac{\dot{E}_{p}}{\dot{E}_{m}}$

$\varepsilon=\frac{1588}{1593}=0,99$

Eficiência exergética maior:

$$
\begin{aligned}
& \varepsilon^{+}=\frac{\dot{E}_{p}+\dot{E}_{l}}{\dot{E}_{m}} \\
& \varepsilon^{+}=\frac{1588+0}{1593}=0,99
\end{aligned}
$$

4.4.5.5 Bomba do líquido saturado do condensador

\subsection{Condições na entrada}

Temperatura na entrada

$T_{e l p}=38,94{ }^{\circ} \mathrm{C}$

Pressão na entrada

$p_{e l p}=6,971 \mathrm{kPa}$

Entalpia na entrada

$h_{e l p}=163,1 \mathrm{~kJ} / \mathrm{kg}$

Entropia na entrada 
$S e l p=0,5582 \mathrm{~kJ} / \mathrm{kgK}$

(EES)

4.4.5.5.2 Balanço de massa

$\dot{m}_{e l p}=191,4 \mathrm{~kg} / \mathrm{s}$

$\dot{m}_{s l p}=\dot{m}_{e l p}$

4.4.5.5.3 Condições na saída

Temperatura na saída

$T_{s l p}=39,17^{\circ} \mathrm{C}$

Pressão na saída

$p_{s l p}=792,2 \mathrm{kPa}$

Entalpia na saída

$h_{s l p}=164 \mathrm{~kJ} / \mathrm{kg}$

Entropia na saída

$S_{s l p}=0,5611 \mathrm{~kJ} / \mathrm{kgK}$

(EES)

\subsection{Balanço de energia}

A potência consumida pela bomba será calculada através da equação (3.88) onde foi admitida uma eficiência de 0,9 para a bomba. A densidade considerada será a do líquido saturado à temperatura de entrada.

$$
\begin{aligned}
& \rho_{l p}=992,6 \mathrm{~kg} / \mathrm{m}^{3} \\
& \eta_{b b}=\frac{\dot{m} l\left(p_{s l}-p_{e l}\right)}{\frac{\dot{W}_{p}}{\rho W_{p}}} \\
& \dot{W}_{p}=\frac{191,4 \cdot(792,2-6,971)}{0,9 \cdot 992,6}=168,2 \mathrm{~kW}
\end{aligned}
$$

\subsection{Análise exergética}

Exergia dos fluxos de massa na entrada:

$e_{e}=\left(h_{e l p}-h_{0 l s}\right)-T_{0}\left(S_{e l p}-s_{0 l s}\right)$

$e_{e l p}=(163,1-65,34)-288,7(0,5582-0,2323)=3,667 \mathrm{~kJ} / \mathrm{kg}$

Exergia dos fluxos de massa na saída:

$$
\begin{aligned}
& e_{s l p}=\left(h_{s l p}-h_{0 l s}\right)-T_{0}\left(s_{s l p}-s_{0 l s}\right) \\
& e_{s l p}=(164-65,34)-288,7(0,5611-0,2323)=3,737 \mathrm{~kJ} / \mathrm{kg}
\end{aligned}
$$

Matéria prima:

$$
\begin{aligned}
& E_{m}=\dot{m}_{e_{l p} e_{e l p}+\dot{W}_{b}} \\
& E_{m}=191,4 \cdot 3,667+168,2=870 \mathrm{~kW}
\end{aligned}
$$

Produtos: 
$E_{p}=\dot{m}_{s p} e_{s l p}$

$E_{p}=191,4 \cdot 3,737=715,2 \mathrm{~kW}$

(Eq. 3.91)

Perdas:

Não há perdas neste componente.

Exergia dissipada:

$\dot{E}_{d}=\dot{E}_{m}-\left(\dot{E}_{p}+\dot{E}_{l}\right)$

$\dot{E}_{d}=870-(715,2+0)=154,8 \mathrm{~kW}$

Eficiência exergética:

$$
\begin{aligned}
& \varepsilon=\frac{\dot{E}_{p}}{\dot{E}_{m}} \\
& \varepsilon=\frac{715,2}{870}=0,82
\end{aligned}
$$

Eficiência exergética maior:

$$
\begin{aligned}
\varepsilon^{+} & =\frac{\dot{E}_{p}+\dot{E}_{l}}{\dot{E}_{m}} \\
\varepsilon^{+} & =\frac{715,2+0}{870}=0,82
\end{aligned}
$$




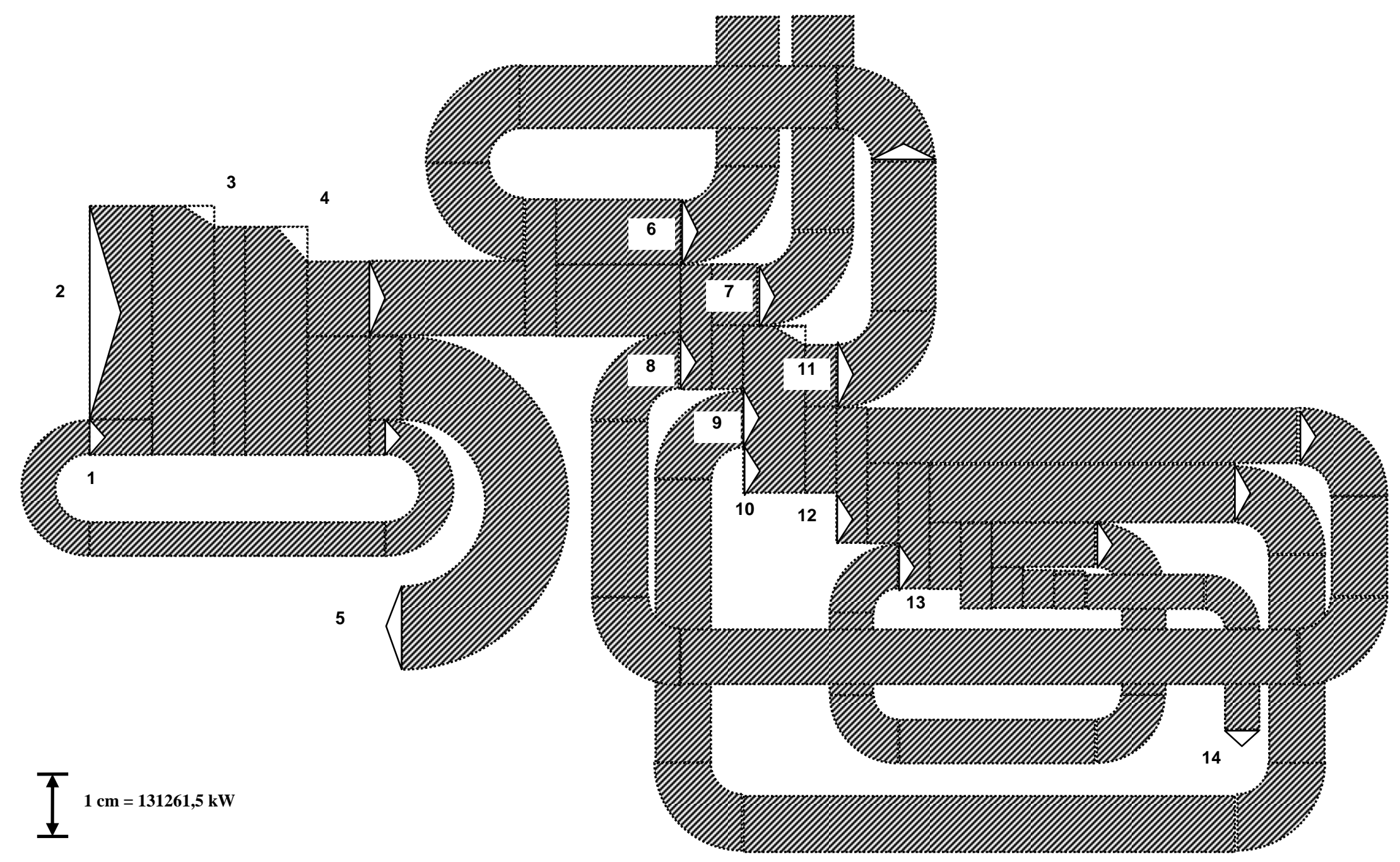

Figura 4.3 - Diagrama de Grasmam para a usina de Uruguaiana (Ciclo Brayton e Trocadores de calor com os gases da exaustão) 
4.5 Síntese da análise do ciclo de Uruguaiana

A seguir foram resumidas as informações obtidas através dos cálculos realizados anteriormente. Os resultados estão dispostos na tabela abaixo:

\begin{tabular}{|c||r|r||r||r|r|r||}
\hline \multicolumn{1}{|c||}{} & \multicolumn{2}{|c|}{ Fluxos de exergia [kW] } & \multicolumn{1}{|c|}{ Eficiência exergética } \\
\cline { 2 - 6 } Componente & Matéria prima & Produtos & Perdas & Destruição & \multicolumn{1}{|c|}{$\mathbf{E}_{\mathbf{f}}$} & \multicolumn{1}{|c|}{$\mathbf{E}_{\mathbf{f}}^{+}$} \\
\hline Compressor & 74624 & 72736 & 0 & 1888 & 0,97 & 0,97 \\
\hline Combustor & 525046 & 481822 & 0 & 43224 & 0,92 & 0,92 \\
\hline Turbina a gás & 476162 & 250713 & 152352 & 73097 & 0,53 & 0,85 \\
\hline Trocador de calor (VC 1-2) & 287060 & 135183 & 150437 & 1440 & 0,47 & 0,99 \\
\hline Trocador de calor (VC 2-3) & 263253 & 128862 & 133458 & 933 & 0,49 & 0,99 \\
\hline Trocador de calor (VC 3-4) & 353452 & 130291 & 183811 & 39350 & 0,37 & 0,89 \\
\hline Trocador de calor (VC 4-5) & 286786 & 112816 & 170140 & 3829 & 0,39 & 0,98 \\
\hline Trocador de calor (VC 5-6) & 263357 & 119945 & 137744 & 5668 & 0,45 & 0,98 \\
\hline Gerador de vapor (HP) & 179505 & 93216 & 80010 & 6279 & 0,52 & 0,96 \\
\hline Trocador de calor (VC 7-8) & 87814 & 8448 & 76901 & 2465 & 0,1 & 0,97 \\
\hline Trocador de calor (VC 8-9) & 87523 & 41761 & 42885 & 2877 & 0,48 & 0,97 \\
\hline Trocador de calor (VC 9-10) & 50166 & 7805 & 40113 & 2248 & 0,15 & 0,95 \\
\hline Gerador de vapor (MP) & 41219 & 4465 & 35435 & 1319 & 0,11 & 0,97 \\
\hline Trocador de calor (VC 11-12) & 46840 & 21900 & 21457 & 3483 & 0,47 & 0,92 \\
\hline Gerador de vapor (LP) & 31056 & 14126 & 15040 & 1890 & 0,45 & 0,94 \\
\hline Trocador de calor (VC 13-14) & 16072 & 9599 & 3296 & 3177 & 0,6 & 0,8 \\
\hline Aquecedor do combustível & 6113 & 4978 & 855,2 & 279,8 & 0,81 & 0,95 \\
\hline Turbina a vapor HP & 269531 & 61500 & 202783 & 5249 & 0,23 & 0,98 \\
\hline Turbina a vapor IP & 256625 & 64045 & 190406 & 2174 & 0,25 & 0,99 \\
\hline Turbina a vapor LP & 201903 & 147793 & 3336 & 50774 & 0,73 & 0,74 \\
\hline Condensador & 35942 & 16681 & 698,4 & 18562 & 0,46 & 0,48 \\
\hline Bomba MP & 472,8 & 470 & 0 & 2,8 & 0,99 & 0,99 \\
\hline Bomba HP & 11079 & 10922 & 0 & 156,7 & 0,98 & 0,98 \\
\hline Bomba de liquido saturado CB & 1593 & 1588 & 0 & 5 & 0,99 & 0,99 \\
\hline Bomba do condensador & 870 & 715,2 & 0 & 154,8 & 0,82 & 0,82 \\
\hline \hline
\end{tabular}

Tabela 4.1 - Síntese da análise exergética do ciclo de Uruguaiana

\subsubsection{Eficiência Total do Ciclo}

Antes de calcular a eficiência total é necessário definir qual a matéria prima e as perdas a serem consideradas para o ciclo total. Será admitida como a matéria prima a energia fornecida pelas fontes externas ao sistema, assim pode-se adotar a energia do ar fornecido e do combustível como a matéria prima.

$$
E_{m s i s}=\dot{m}_{e a r} \cdot e_{e a r}+\dot{m}_{e c b} \cdot e_{e c b}
$$

Como esse dado foi anteriormente calculado na análise do combustor, será reescrito o valor da exergia obtida para cada substância

$$
E_{m_{s i s}}=431,5 \cdot 168,6+10,23 \cdot 47076=554338 \mathrm{~kW}
$$

Como existem dois blocos iguais que geram potência separadamente, tem-se que a matéria prima total corresponde ao valor calculado multiplicado por dois.

$$
E_{m s i s t}=2 \cdot 554338=1108676 \mathrm{~kW}
$$


As perdas poderão ser interpretadas pelo mesmo critério e serão admitidas como a energia que deixa o ciclo através da saída dos gases para a atmosfera. Essas ocorrem exatamente no volume de controle entre os pontos 13 e 14 do compartimento de fluxo dos gases de exaustão da turbina a gás.

$$
\begin{aligned}
& \dot{E}_{l s i s}=\dot{m}_{s C O 2} \cdot e_{S C O 2}+\dot{m}_{S H 2 O} \cdot e_{S H 2 O}+\dot{m}_{S N 2} \cdot e_{S N 2}+\dot{m}_{s O 2} \cdot e_{S O 2} \\
& E_{l s i s}=33,59 \cdot 5,793+27,51 \cdot 12,79+329,6 \cdot 7,334+51,29 \cdot 6,478=3926 \mathrm{~kW}
\end{aligned}
$$

Da mesma forma feita para o cálculo da matéria prima, deve-se multiplicar o resultado anterior por dois para obter-se a perda total.

$$
E_{l s i s t}=2 \cdot 3926=7852 \mathrm{~kW}
$$

Agora somando toda a exergia dissipada em cada componente, tem-se a exergia total dissipada. Neste caso é necessário observar que não haverá duplicidade de ocorrência deste tipo de exergia em todos os componentes pois já foi informado anteriormente que há somente um condensador e três turbinas a vapor que recebem fluxos de vapor gerados nos dois blocos de turbina a gás. Assim, tem-se que deverá ser duplicada e exergia dissipada nos componentes do ciclo a exceção das turbinas a vapor, condensador e bomba do condensador.

Utilizando a equação (2.32) pode-se calcular a eficiência total do ciclo onde termo $k$ representa cada componente analisado e o segundo membro do lado direito representa a exergia total dissipada.

$$
\varepsilon_{i s}=1-\sum_{k}\left[\frac{\dot{E}_{m}^{k}}{\dot{E}_{m}}\left(1-\varepsilon_{k}^{+}\right)\right]-\frac{\dot{E}_{l}}{\dot{E}_{m}}
$$

Observando o que foi comentado anteriormente a respeito da ocorrência de duplicidade de componentes, foi obtido o seguinte resultado para a eficiência total do sistema.

$$
\varepsilon_{s i s}=1-\frac{464136+7852}{1108676}=0,57
$$

\subsection{Representação do ciclo por diagramas}

Uma forma de representação do ciclo muito interessante do ponto de vista exergético é a utilização dos diagramas de Grasmam. Esses diagramas expressam quantitativamente o nível de exergia que atravessa um volume de controle e proporciona uma visão mais ampla e global da ocorrência de irreversibilidades, perdas de exergia e da geração de potência útil no sistema. As figuras 4.3 e 4.4 representam os diagramas de Grasmam para o ciclo de Uruguaiana. Para auxiliar a interpretação foi definida uma relação entre a escala vertical da figura e o valor correspondente em unidades de exergia em $\mathrm{kW}$. Cada ponto identificado através de um número será descrito a seguir: 


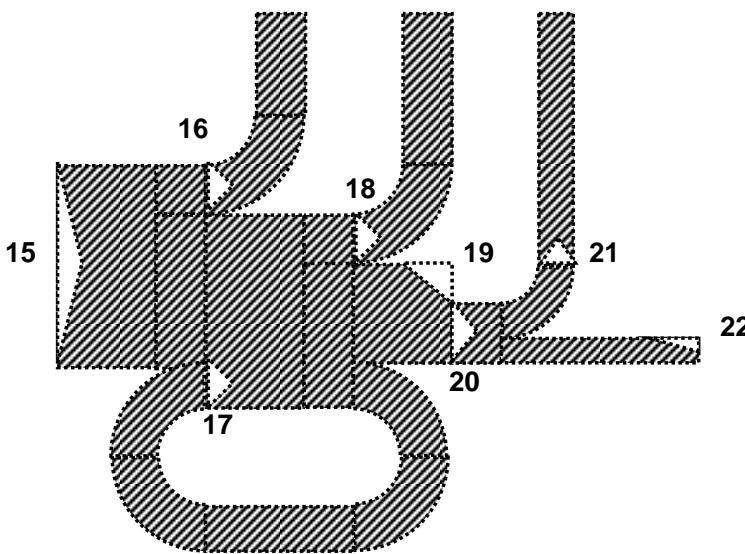

Figura 4.4 - Diagrama de Grasmam para a usina de Uruguaiana (Turbinas a vapor e condensador)

1 - Potência fornecida ao compressor

2 - Exergia do combustível na entrada

3 - Exergia dissipada no combustor

4 - Exergia dissipada na turbina a gás

5 - Potência de saída da turbina

6 - Exergia do vapor em alta pressão ( $3^{\circ}$ passo)

7 - Exergia do vapor em média pressão ( $2^{\circ}$ passo $)$

8 - Exergia do vapor em média pressão $\left(1^{\circ}\right.$ passo $)$

9 - Exergia do vapor em alta pressão ( $1^{\circ}$ passo $)$

10 - Exergia da queima suplementar

11 - Exergia do vapor em alta pressão $\left(2^{\circ}\right.$ passo $)$

12 - Exergia do vapor em média pressão vindo da turbina a vapor

13 - Exergia do vapor em baixa pressão

14 - Exergia dos gases na saída para a atmosfera (Perdas)

15 - Exergia na entrada da turbina a vapor de alta pressão

16 - Potência fornecida pela turbina de alta pressão

17 - Vapor de saída da turbina de média pressão

18 - Potência fornecida pela turbina de média pressão

19 - Exergia dissipada na turbina de baixa pressão

20 - Exergia na saída da turbina de baixa pressão

21 - Potência fornecida pela turbina de baixa pressão

22 - Exergia dissipada no condensador

\subsection{Considerações finais}

Em uma observação mais pontual pode-se observar que as menores eficiências ocorrem nos trocadores de calor. Isso demonstra que este tipo de componente é o que apresenta as maiores limitações em relação ao aproveitamento da energia pois como se sabe as trocas de calor ocorrem através de gradientes finitos de temperatura que são diretamente proporcionais ao aumento das irreversibilidades.

Os componentes que apresentam a maior dissipação de exergia são a Turbina a Gás, Turbina a vapor de baixa pressão e o Combustor respectivamente. Geralmente os processos reativos são os maiores responsáveis pela dissipação, entretanto no ciclo de Uruguaiana esses processos apresentaram-se bastante satisfatórios. Isso ocorre em decorrência de dispositivos e recursos tecnológicos de que se dispõe atualmente. Isso proporciona uma alta eficiência da queima do combustível, com um excelente aproveitamento da energia disponível. No caso da usina de Uruguaiana pode-se citar a utilização do resfriamento evaporativo na entrada do compressor e o dispositivo de redução da emissão de gases como $\mathrm{NO}_{\mathrm{X}}$ e $\mathrm{CO}$. 
Foi realizado o estudo de um ciclo combinado em operação no Brasil baseado em metodologias de análise exergética onde foram obtidos resultados que proporcionaram uma visão global da energia gerenciada pelo ciclo combinado.

O sistema analisado apresentou uma alta eficiência do ponto de vista termodinâmico pois proporcionou um alto rendimento exergético, ou seja, um alto aproveitamento da energia disponível.

Os resultados obtidos podem ser estudados com mais detalhe pois a ausência de algumas informações levou a considerações que poderiam ser reavaliadas.

A utilização do programa EES proporcionou um resultado satisfatório pois permitiu uma criação de resultados que poderão ser utilizados e adequados à diferentes necessidades através de arquivos de dados.

Alguns modelos propostos apresentaram-se limitados pois não levaram em consideração a utilização de recursos adicionais que existem no ciclo analisado. Isso levou a adequações que poderiam afetar os resultados a serem atingidos.

Ao adotar o modelo de reação do metano para a combustão envolvida no ciclo foram suprimidas algumas contribuições de energia que poderiam ocorrer por conta de outras reações que ocorrem na combustão tendo em vista que o gás fornecido não é constituído plenamente de metano.

Como análise preliminar do ciclo, este trabalho poderia ser avaliado de modo a elaborar um estudo mais aprofundado e estender este tipo de análise aos demais ciclos em operação no Brasil.

Por fim, o trabalho aqui desenvolvido proporcionou uma grande oportunidade de aplicação do conhecimento da termodinâmica em uma situação real de operação de um ciclo termodinâmico. 
BEJAN, A. 1988 - Advanced Engineering Thermodynamics - John Wiley \& Sons, INC. -New York

BEJAN, A. ;TSATSARONIS, G. ;MORAN, M. 1996 - Dedign of Thermal Systems - Jhon Wiley \& Sons, INC. - New York

MORAN, M. J., SCIUBBA, E., 1994 - Exergy Analysis: Principles and Practice - Journal of Engineering for Gas Turbines and Power - vol.116, pp.285-290

COHEN, H. ; ROGERS, G. F. C. ; SARAVANAMUTO, H.I.H. - 1996 - Gas Turbine Theory Editora Longman - England; $4^{\mathrm{a}}$ edição

CONSONNI, S. ; LARSON, E.D. - 1996 - Biomass-Gasifier/Aeroderivative Gas Turbine Combined Cycles - Part A: Technologies and Performace Modeling - Journal of Engineering for Gas Turbine and Power, vol. 118, pp 507-515

KORAKIANITIS, T. ; WILSON, D. G. - 1994 - Models for Predicting lhe Performace of Brayton Cycle Engines - Journal ofEngineering for Gas Turbines and Power - vol.116, pp. 381 - 388

LEFEBVRE, A.R. 1995 - The Role of Fuel Preparation in Low-emission Combustion - Journal of Engineering for Gas Turbines and Power - vol.117, pp. 617-654

SIPOT - Sistema de informações do potencial hidrelétrico Brasileiro - Elaborado por Eletrobrás, GCPS (Grupo coordenador de planejamento de sistema elétricos) e CGOI (Grupo coordenador para operação interligada) - janeiro/2000.

VAN WYLEN, G. J.; SONNTAG, R.E. - 1976 - Fundamentos da termodinâmica clássica Editora Edgard Blücher Ltda - São Paulo - 2a Edição

LOGRADO, C. L.; 2000 - Análise e otimização de turbinas a gás e ciclos combinados Dissertação de mestrado - Publicação DM 44, Departamento de Engenharia Mecânica, Universidade de Brasília, DF, 219p

KOTAS, T. J. (Tadeusz Jozef), 1995 - The exergy method of thermal plant analysis - KRIEGER PUBLISHING COMPANY - Malabar, Florida 32950 


\section{A1 - ALGORITMO PARA CÁLCULO DA TEMPERATURA DOS GASES NA SAÍDA DO COMBUSTOR}

De acordo com o item 4.3.2.5 a temperatura de saída dos gases após a reação no combustor será obtida considerando que todos as substâncias se encontrarão à mesma temperatura. Para o cálculo da temperatura será considerado o balanço de energia para o combustor

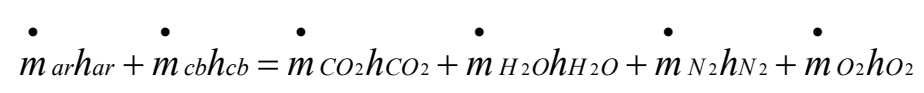

Neste caso será considerada a combustão do metano com ar e metano como reagentes e $\mathrm{CO}_{2}, \mathrm{H}_{2} \mathrm{O}, \mathrm{N}_{2}$ e $\mathrm{O}_{2}$ como produtos da reação.

Como entrada para o processo iterativo será admitida a temperatura adiabática de chama. Assim são obtidas as propriedades de cada substância e em seguida calcula-se o balanço de energia para a temperatura especificada. Caso o balanço seja atendido encontra-se a temperatura de saída e o processo é encerrado.

A seguir será demonstrado o processo através de passos a serem executados:

1 - Dados: $T_{g}{ }^{*}=T_{\text {chama }}$ (Admite-se a temperatura de entrada como $T_{\text {chama }}$ ).

2- $\quad$ Propriedades termodinâmicas: $h_{i}, s_{i},\left(i=\mathrm{CO}_{2}, \mathrm{H}_{2} \mathrm{O}, \mathrm{N}_{2}\right.$ e $\left.\mathrm{O}_{2}\right)$, obtêm-se as propriedades de cada substância na saída do combustor.

3- $\quad$ Calcula-se o balanço de energia.

4- $\quad$ Calculo do erro pela diferença entre a energia na entrada e na saída:

$$
\begin{aligned}
& E_{e}=\dot{m}_{a r} h_{a r}+\dot{m}_{c b} h_{c b} \\
& E_{s}=\dot{m}_{\mathrm{CO}_{2}} h_{\mathrm{CO}_{2}}+\dot{m}_{\mathrm{H}_{2} \mathrm{O}_{\mathrm{H}_{2} \mathrm{O}}+\dot{m}_{\mathrm{N}_{2}} h_{\mathrm{N}_{2}}+\dot{m_{\mathrm{O}}} h_{\mathrm{O}}} \\
& \text { Erro }=E_{s}-E_{e}
\end{aligned}
$$

se o erro for menor do que a tolerância especificada o valor de $T_{g}{ }^{*}$ é a temperatura de saída dos gases. Caso contrário faz-se $T_{g}=T_{g}^{*}-i n c$ e repete-se os passos 2,3 e 4 onde inc é o incremento a ser considerado.

Usando-se esse algoritmo a temperatura de saída dos gases é calculada. 